\title{
Spontaneous chiral symmetry breaking in holographic soft wall models
}

\author{
Alfonso Ballon-Bayona, ${ }^{1, *}$ Luis A. H. Mamani, ${ }^{2, \dagger}$ and Diego M. Rodrigues $\odot^{3, \neq}$ \\ ${ }^{1}$ Instituto de Física, Universidade Federal do Rio de Janeiro, \\ 21.941-972 Rio de Janeiro, Rio de Janeiro, Brazil \\ ${ }^{2}$ Centro de Ciências Exatas Naturais e Tecnológicas, Universidade Estadual da Região Tocantina do \\ Maranhão, Rua Godofredo Viana 1300, 65901- 480 Imperatriz, Maranhão, Brazil \\ ${ }^{3}$ Instituto de Física Teórica, Universidade Estadual Paulista, \\ R. Dr. Bento T. Ferraz 271, Bl. II, São Paulo 01140-070, São Paulo, Brazil
}

(Received 11 August 2021; accepted 8 November 2021; published 27 December 2021)

\begin{abstract}
We investigate nonlinear extensions of the holographic soft wall model proposed by Karch et al. [Phys. Rev. D 74, 015005 (2006)] including nonminimal couplings in the five-dimensional action. The nonminimal couplings bring a new parameter $a_{0}$ which controls the transition between spontaneous and explicit symmetry breaking near the limit of massless quarks (the chiral limit). In the physical region (positive quark mass), we show that above a critical value of the parameter $a_{0}$ the chiral condensate $\langle\bar{q} q\rangle$ is finite in the chiral limit, signifying spontaneous chiral symmetry breaking. This result is supported by the lightest states arising in the spectrum of the pseudoscalar mesons, which become massless in the chiral limit and are therefore intrepreted as Nambu-Goldstone bosons. Moreover, the decay constants of the pseudoscalar mesons also support this conclusion, as well as the Gell-Mann-Oakes-Renner relation satisfied by the lightest states. We also calculate the spectrum of scalar, vector, and axial-vector mesons with their corresponding decay constants. We describe the evolution of masses and decay constants with the increasing of the quark mass, and for the physical mass, we compare our results against available experimental data. Finally, we do not find instabilities in our model for the physical region (positive quark mass).
\end{abstract}

DOI: 10.1103/PhysRevD.104.126029

\section{INTRODUCTION}

The investigation of spontaneous symmetry breaking (SSB) is a very interesting subject, essential in condensed matter and particle physics contexts. It is related with the symmetry of the quantum field theory (QFT) action and the vacuum solution of the theory. It is believed that SSB is realized only in systems with infinite degrees of freedom. An example of the realization of SSB in QFT is the emergence of Nambu-Goldstone bosons in the theory, which are related to the breaking of a global symmetry breaking via the Goldstone theorem [1]. Moreover, in such systems, the vacuum expectation value does not have the same symmetry as the action. In particle physics, the pions are an example of pseudo Nambu-Goldstone bosons. In turn, QCD has an approximate $U(2)_{L} \times U(2)_{R}$ symmetry in the limit of light quark masses, considering just up and

\footnotetext{
*aballonb@if.ufrj.br

†uis.mamani@uemasul.edu.br

¥diego.m.rodrigues@unesp.br
}

Published by the American Physical Society under the terms of the Creative Commons Attribution 4.0 International license. Further distribution of this work must maintain attribution to the author(s) and the published article's title, journal citation, and DOI. Funded by SCOAP ${ }^{3}$. down quarks. The symmetry breaking leads to $U(2)_{L} \times$ $U(2)_{R} \rightarrow U(2)_{V}$, and it gives rise to pseudo-NambuGoldstone bosons; see, for instance, Refs. [2,3]. This symmetry breaking occurs in the low energy regime of $\mathrm{QCD}$, or strong coupling regime, where perturbative techniques are not reliable anymore. However, there are effective theories that one may use in this regime, like QCD sum rules, chiral effective theories, Dyson-Schwinger equations, or even numerical methods introduced by lattice QCD. In the past few years, lattice QCD became a powerful computational tool for extracting information of QCD at low energies. Nevertheless, there are still difficulties and limitations to be overcome for this fascinating subject; for an investigation of chiral symmetry breaking in lattice QCD with two light flavors, see, for instance, Ref. [4].

An alternative theoretical framework for investigating strongly coupled systems was provided by the anti-de Sitter (AdS)/conformal field theory (CFT) correspondence, proposed at the end of the 1990s [5]. Using as a guide the holographic dictionary [6,7], one may map any operator of a strongly coupled conformal field theory into its dual classical field in the gravitational side of the duality living in an AdS space with one dimension greater than the space where the conformal field theory lives. In the case of four dimensions, one may break the conformal symmetry and 
introduce symmetries known to be present in QCD so that the original conformal field theory becomes more similar to QCD. This is the motivation behind a bottom-up approach that led to the construction of phenomenological AdS/QCD models known as the hard wall model $[8,9]$ and the soft wall model [10]. There has been a lot of progress on this approach; see, for instance, Refs. [11-27] and references therein. However, it is worth pointing out that the gravitational background is not obtained solving the Einstein equations; this means that the metric is fixed being AdS. Nevertheless, one may consider this naive approximation as a toy model to gain intuition. A more consistent version of this approach takes into consideration the gravitational background obtained solving the Einstein equations; see, for instance, Refs. [28-34] and the references therein. The bottom-up approach is complementary to the top-down approach, whose goal is to find string theory duals to strongly coupled QCD-like theories; see, for instance, Refs. [35-39]. For a review of the top-down approach, see, for instance, Refs. [40-42].

One of the aims of this work is the investigation of spontaneous chiral symmetry breaking within holographic models for QCD. The first attempts to describe chiral symmetry breaking in the bottom-up approach were proposed in Refs. [8,9], in which the quark mass operator is mapped to a five-dimensional (5D) tachyonic field. These bottom-up models as well as their top-down counterparts, see, e.g., Refs. [38,43,44], have provided very useful insights for the construction of more realistic holographic models for QCD. There has been recent progress on the description of spontaneous chiral symmetry breaking in bottom-up holographic QCD models inspired by string theory [45-48] and its relation to the violation of the Breitenlohner-Freedman bound [49,50].

In this paper, we restrict ourselves to the holographic soft wall model proposed in Ref. [10], in which the dilaton is quadratic in the radial coordinate leading to meson masses organized in linear trajectories in the excitation number, i.e., $m_{n}^{2} \sim n$. It is important to point out that the original version of the linear soft wall model does not describe spontaneous chiral symmetry breaking [12]. It was suggested in Ref. [10] that the addition of nonlinear terms in the action for the tachyonic field would allow for a nonlinear description of chiral symmetry breaking that may be compatible with spontaneous symmetry breaking. Following this suggestion, the authors of Ref. [13] built a phenomenological model compatible with spontaneous symmetry breaking in the sense that the quark condensate is finite in the chiral limit.

In our opinion, this model provides good results, but there are a few caveats that we point out:

(i) The chiral condensate is given as an input instead of arising dynamically from solving the tachyon differential equation. (ii) The dilaton is nonmonotonic and negative near the AdS boundary.

(iii) An instability arises in the scalar sector first observed in Ref. [21]; see also Ref. [31].

Following the ideas of Refs. [45-47], the authors of Ref. [51] showed that the nonlinear extension of the original soft wall model [10] based on a quadratic dilaton and a Higgs-like potential for the tachyonic field indeed allows for a nonlinear realisation of chiral symmetry breaking. However, it was shown in Ref. [51] that the chiral condensate still vanishes in the massless limit and therefore chiral symmetry breaking is explicit.

An attempt to describe spontaneous chiral symmetry breaking in the soft wall model was motivated by the discussion on the sign of an effective dilaton field, responsible for conformal symmetry breaking and the mass generation in the infrared. A negative dilaton profile was proposed in Refs. [52,53] as an alternative to describe the hadronic spectrum and spontaneous chiral symmetry breaking. However, there has been a debate about the phenomenological consequences of the negative dilaton profile. It has been shown in Ref. [54] that the negative dilaton drives to the emergence of an unphysical massless scalar state in the vectorial sector. Besides that, the negative dilaton leads to unphysical masses for higher spin mesons [54]. Motivated by this very interesting discussion, the authors of Ref. [19] suggested that an interpolation function between an effective negative dilaton in the UV region and positive in the IR region might provide a description of spontaneous chiral symmetry breaking. Nevertheless, the authors of that paper did not calculate the spectrum of the pseudoscalar mesons at zero temperature and proved the emergence of the pions as Nambu-Goldstone bosons. In this paper, we work on the ideas of Ref. [19] and reinterpret them in terms of nonminimal dilaton couplings in the 5D action. Following the techniques used in Ref. [51], we solve the tachyon differential equation and find that the new parameter $a_{0}$ controlling the nonminimal couplings allows us to describe spontaneous chiral symmetry breaking in the chiral limit. We show explicitly that, above a critical value for $a_{0}$, the chiral condensate is nonzero in the chiral limit signaling spontaneous chiral symmetry breaking and that this result is consistent with the emergence of massless modes in the pseudoscalar sector, i.e., the pions as Nambu-Goldstone bosons. As a check of consistency, we also calculate the decay constants and show that the lightest states in the pseudoscalar sector satisfy the Gell-Mann-Oakes-Renner (GOR) relation.

This paper is organized as follows. Section II is a short review of the holographic soft wall model and its nonlinear extension. In Sec. III, we present the nonlinear extension of the soft wall model including the nonminimal couplings. We consider two interpolating functions for those couplings, models of type I and models of type II. We solve the tachyon differential equation in its 
asymptotic regions, and then we solve it numerically. Near the chiral limit, we describe the transition between spontaneous and explicit chiral symmetry breaking driven by the parameter $a_{0}$ characterizing the nonminimal couplings. The free parameters of our model are fixed at the end of that section. In Sec. IV, we expand the fivedimensional action up to second order in the fields in order to get the equation of motions describing the vector, scalar, axial-vector, and pseudoscalar mesons. The corresponding spectrum is calculated and compared against results available in the literature. In Sec. V, we calculate the decay constants corresponding to vector, scalar, axialvector, and pseudoscalar mesons, showing how they are related to the normalization constants of the wave functions. At the end of the section, we derive the GOR relation. We present our conclusions in Sec. VI. Additional details about the numerical procedure and results are displayed in Appendix A. Meanwhile, in Appendix B, we present the Kaluza-Klein expansion. Details about the derivation of the decay constants are presented in Appendix C. Details of the masses and decay constants in the linear soft wall model are reviewed in Appendix D. A short discussion about the linear soft wall model with negative dilaton is presented in Appendix E, while the nonlinear extension is investigated in Appendix F. Finally, we derive the GOR relation using a toy model in Appendix G.

\section{SOFT WALL MODELS WITH MINIMAL DILATON COUPLINGS}

\section{A. Original soft wall model}

One of the seminal papers considering a bottom-up approach in holographic QCD is the soft wall model [10]. In Ref. [10], the authors introduced a scalar field, the dilaton, in a $5 \mathrm{~d}$ anti-de Sitter space in order to incorporate effects of conformal symmetry breaking and generate an infrared mass gap. They showed that a dilaton growing quadratically in the radial coordinate far from the boundary leads to an approximate linear behavior of the mass spectrum, i.e., $m^{2} \propto n$. It is worth pointing out that the dilaton proposed in Ref. [10] was not obtained solving the Einstein equations. Then, one might think that the confinement properties introduced by the dilaton field are "artificial." However, it has been shown that the quadratic behaviour proposed in Ref. [10] is in fact the right asymptotic behavior for the dilaton in confining holographic QCD backgrounds found by solving the Einsteindilaton equations [29]; see also Ref. [32].

Thus, we consider that the soft wall model as a first step for a realistic description is good enough, at least to extract general properties of the dual field theory. Thus, having identified the fields in the gravitational side of the duality, one can write the five-dimensional action, which is given by

$$
\begin{aligned}
S= & -\int d^{5} x \sqrt{-g} e^{-\Phi} \operatorname{Tr}\left[\left|D_{m} X\right|^{2}+m_{X}^{2}|X|^{2}+\frac{1}{4 g_{5}^{2}} F_{m n}^{(L) 2}\right. \\
& \left.+\frac{1}{4 g_{5}^{2}} F_{m n}^{(R) 2}\right]
\end{aligned}
$$

where $\Phi=\Phi(z)$ is the dilaton field, $X$ is the bifundamental scalar field (or tachyonic field) dual to the quark mass operator $\bar{q} q, m_{X}^{2}=-3$ the mass of the tachyonic field. The field strength $F_{n m}^{(L / R)}$ and covariant derivative $D_{m} X$ are defined by

$$
\begin{aligned}
F_{m n}^{(L / R)} & =\partial_{m} A_{n}^{(L / R)}-\partial_{n} A_{m}^{(L / R)}-i\left[A_{m}^{(L / R)}, A_{n}^{(L / R)}\right], \\
D_{m} X & =\partial_{m} X-i A_{m}^{(L)} X+i X A_{m}^{(R)}
\end{aligned}
$$

where $A_{n}^{(L / R)}$ represent the gauge fields. As usual in the soft wall model approach, the 5D metric $g_{m n}$ is the AdS spacetime in Poincare coordinates. The 5D coupling in the vectorial sector is fixed as $g_{5}^{2}=12 \pi^{2} / N_{c}$, with $N_{c}$ the number of colors, in order to reproduce the perturbative QCD result for the current correlators at small distances [8]. The action (1) describes the breaking of the $S U\left(N_{f}\right)_{L} \times S U\left(N_{f}\right)_{R}$ gauge symmetry due to the presence of a nonzero tachyonic field $X$. The generators of the $s u\left(N_{f}\right)$ algebra are normalized as $\operatorname{Tr}\left(T^{a} T^{b}\right)=\delta^{a b} / 2$, and we will focus on the two-flavor case $N_{f}=2$.

A very nice feature of the soft wall model is the possibility of describing approximate linear trajectories for the meson spectrum, i.e., $m_{n}^{2} \sim n$, by simply imposing an asymptotic quadratic behavior for the dilaton field, i.e., $\Phi(z)=\phi_{\infty} z^{2}$ at large $z$ (far from the boundary). As realized in Ref. [10], if the dilaton quadratic ansatz is taken for all $z$, the effective Schrödinger potential of the field perturbations dual to the mesons takes the form of an harmonic oscillator with orbital momentum, and the linear behavior $m_{n}^{2} \sim n$ becomes exact. Appendix D provides a short review of the results for vector and scalar mesons in the (linear) soft wall model.

The (linear) soft wall model (1) leads to a linear differential equation for the tachyonic field $X$. Regularity of the action in the infrared region (large $z$ ) fixes the source and vacuum expectation value (VEV) coefficients in the UV, and it turns out that the chiral condensate is proportional to the quark mass and vanishes in the chiral limit.

\section{B. Nonlinear soft wall model}

Nonlinear extensions of the soft wall model have been investigated in the literature; see, for instance, Refs. [13,19,51]. The nonlinearity is carried out by the tachyon potential. Then, the action is a modified version of (1), 


$$
\begin{aligned}
S= & -\int d^{5} x \sqrt{-g} e^{-\Phi} \operatorname{Tr}\left[\left|D_{m} X\right|^{2}+V(|X|)+\frac{1}{4 g_{5}^{2}} F_{m n}^{(L) 2}\right. \\
& \left.+\frac{1}{4 g_{5}^{2}} F_{m n}^{(R) 2}\right]
\end{aligned}
$$

where the non-Abelian field strengths $F_{m n}^{(L / R)}$ and covariant derivative $D_{m} X$ are given by (2), while the tachyon potential has the Higgs form

$$
V(|X|)=m_{X}^{2}|X|^{2}+\lambda|X|^{4},
$$

where $\lambda$ is a free parameter. The $5 \mathrm{D}$ metric is again the AdS spacetime in Poincaré coordinates. The form of the Higgs potential (4) is motivated by the fact that it provides a minimal description of spontaneous breaking of a local symmetry in the standard model and may be possibly extended to AdS space in order to describe the gravity dual of the spontaneous breaking of a global symmetry. The potential in (4) seems also a good ansatz if we want to build a holographic realization of the Coleman-Witten theorem [55].

The action in (3) was used in the phenomenological model of Ref. [13] with $\lambda<0$ for the Higgs potential (4). Instead of solving for the tachyonic field for a given dilaton field, an inverse method was proposed: given a tachyonic field compatible with spontaneous chiral symmetry breaking, the dilaton field was reconstructed. The ansatz for the tachyonic field in Ref. [13] is singular at large $z$, and the effective dilaton field found in Ref. [13] is quadratic at large $z$ (far from the AdS boundary) but becomes nonmonotonic and negative as $z$ approaches zero (near the AdS boundary). In Ref. [51], the same action in (3) was considered for a quadratic dilaton field, and the chiral condensate was found dynamically by solving the tachyonic field differential equation. The main result in Ref. [51] was that the only nontrivial solution for the tachyonic field is regular far from the boundary and leads to a chiral condensate growing nonlinearly with the quark mass. However, it turns out that this regular solution vanishes in the chiral limit and therefore spontaneous chiral symmetry breaking is absent. It was found in Ref. [51] that for the case of $\lambda>0$ the model provides very interesting results in the limit of heavy quarks. The meson masses and decay constants obtained in the model for the axial and pseudoscalar sector display a behavior qualitatively similar to the one found in perturbative QCD.

Note that the tachyonic field in (3) couples minimally with the dilaton, i.e., the factor $e^{-\Phi}$ in front of the trace, depends on the dilaton field only. In the next section, we shall investigate how to generalize the form of this dilaton coupling. We will see that keeping the minimal dilaton coupling only at large $z$ (where $\Phi$ is large) but deviating from it at small $z$ (where $\Phi$ is small) it is possible to describe spontaneous chiral symmetry breaking in the chiral limit.

\section{NONLINEAR SOFT WALL MODELS WITH NONMINIMAL DILATON COUPLINGS}

The gravitational action (3) can be generalized considering two nonminimal couplings in the form

$$
\begin{aligned}
S= & -\int d^{5} x \sqrt{-g} \operatorname{Tr}\left\{e^{-a(\Phi)}\left[\left|D_{m} X\right|^{2}+V(|X|)\right]\right. \\
& \left.+\frac{e^{-b(\Phi)}}{4 g_{5}^{2}}\left[F_{m n}^{(L) 2}+F_{m n}^{(R) 2}\right]\right\} .
\end{aligned}
$$

The fields of this action are the same as presented previously, and the difference in relation to the previous action is the addition of the functions: $e^{-a(\Phi)}$, and $e^{-b(\Phi)}$, which are in principle nontrivial functions of the dilaton field.

Having specified the gravitational action describing the quarks of the dual field theory, we now consider the gravitational background, which is characterised by a Poincaré invariant metric

$$
d s^{2}=e^{2 A_{s}(z)}\left(-d t^{2}+d \vec{x}^{2}+d z^{2}\right),
$$

where $A_{s}(z)$ is the warp factor in the string frame. As usual in soft wall models, we fix the warp factor and dilaton as

$$
A_{s}(z)=-\ln \left(\frac{z}{\ell}\right), \quad \Phi(z)=\phi_{\infty} z^{2} .
$$

That is, the 5D metric is AdS in Poincare coordinates, and the dilaton is quadratic in the radial coordinate $z$. The parameter $\ell$ denotes the AdS radius, and from here on, we set $\ell=1$. Note that $\phi_{\infty}$ is a parameter with units of energy squared. The original soft wall model corresponds to the particular case $a(\Phi)=b(\Phi)=\Phi$. For that particular case, as shown in Ref. [10], a quadratic dilaton is sufficient to guarantee a linear behavior for the meson Regge trajectories. In this work, we consider more general dilaton couplings $a(\Phi)$ and $b(\Phi)$. We consider for simplicity the case $N_{f}=2$ where the model describes the gauge symmetry breaking $S U(2) \times S U(2) \rightarrow S U(2)$, which is the gravity dual of chiral symmetry breaking in QCD with two flavors.

\section{A. Background field equations}

For the background fields, we take a Lorentz invariant ansatz,

$$
A_{m}^{(L / R)}=0, \quad 2 X(z)=v(z) \mathbb{I}_{2 \times 2} .
$$

Under this ansatz, the action takes the one-dimensional (1D) form 


$$
S_{0}=-V_{4} \int d z e^{3 A_{s}-a}\left[\frac{1}{2}\left(\partial_{z} v\right)^{2}+e^{2 A_{s}} U(v)\right],
$$

where

$$
U(v)=\frac{m_{X}^{2}}{2} v^{2}+\frac{\lambda}{8} v^{4}
$$

is the potential for the 1D function $v(z)$ and $V_{4}=\int d^{4} x$. We remind the reader that $A_{s}$ and $a$ depend only on $z$. The critical points of the potential are obtained solving the equation $\frac{d U(v)}{d v}=0$, with $m_{X}^{2}=-3$, and we get three solutions, two of them corresponding to minimum and the other to the trivial solution (the local maximum)

$$
v= \pm \sqrt{\frac{6}{\lambda}}, \quad v=0
$$

The Euler-Lagrange equation for $v(z)$ can be written as

$$
\left[\partial_{z}+\left(3 A_{s}^{\prime}-a^{\prime}\right)\right] \partial_{z} v-e^{2 A_{s}} \frac{d U}{d v}=0
$$

where ' denotes $d / d z$. Note that this equation simplifies when evaluated at the critical points where the derivative of the potential is zero,

$$
\begin{gathered}
\left(\ln v^{\prime}(z)\right)^{\prime}+\left(3 A_{s}-a\right)^{\prime}=0, \\
\rightarrow \quad v=C_{0}+D_{0} \int_{0}^{z} e^{-\left(3 A_{s}(x)-a(x)\right)} d x .
\end{gathered}
$$

This means that the tachyon has at least a constant as a solution and the other solution is divergent. Plugging this solution in (12), considering the regular solution, we get an expression for $C_{0}$ whose solutions are $C_{0}= \pm \sqrt{6 / \lambda}$ and $C_{0}=0$. If we look for solutions in the IR, the corrections to $C_{0}$ can be calculated considering corrections in the form $\mathcal{O}\left(z^{-1}, z^{-2}, \cdots\right)$.

In turn, considering $A_{s}=-\ln z$ and the potential $U(v)$ given by (10), the tachyon field equation (12) takes the form

$$
\left[z^{2} \partial_{z}^{2}-\left(3+z a^{\prime}\right) z \partial_{z}-m_{X}^{2}\right] v-\frac{\lambda}{2} v^{3}=0 .
$$

Another interesting property of this differential equation is the scaling symmetry in $\sqrt{\lambda}$; this means that the equation is the same under the transformation $\sqrt{\lambda} v \rightarrow \tilde{v}$. One may take advantage of this property. For example, we may solve the differential equation for $\tilde{v}$; then, we get the solution for $v$ scaling $\tilde{v}$, i.e., using the relation $v=\tilde{v} / \sqrt{\lambda}$. We will see the applicability of this symmetry in the next section.

\section{B. Interpolations for the dilaton coupling}

So far, we have the differential equation for the tachyon; it is lacking the explicit form of the nonminimal coupling $a(\Phi)$, and $b(\Phi)$. In the following, we motivate our choices for the functions $a(\Phi)$ and $b(\Phi)$.

The nonminimal couplings $a(\Phi)$ and $b(\Phi)$ were originally considered to be equal and both interpreted as an effective dilaton field $\tilde{\Phi}=a(\Phi)=b(\Phi)$ so that the effective dilaton field $\tilde{\Phi}$ couples minimally to the tachyon and gauge fields. An effective negative dilaton field $\tilde{\Phi}$ quadratic in the radial coordinate allows for a description of spontaneous chiral symmetry breaking; see Appendix E for a short review. However, the authors of Ref. [54] showed that a nonphysical state arises in the vectorial sector when the dilaton remains negative in the IR. This means that the dilaton must be positive in the IR in order to avoid nonphysical states. We believe this was the motivation of the authors of Ref. [19] for building an interpolation for the effective dilaton field, whose asymptotic form reduces to a negative $\tilde{\Phi}$ (quadratic in the radial coordinate) in the UV and a positive $\tilde{\Phi}$ in the IR (also quadratic in the radial coordinate). Following these considerations, we impose that the nonminimal coupling reduces to the minimal coupling in the IR regime, namely,

$$
a(\Phi \rightarrow \infty)=\Phi
$$

We remind the reader that in our framework the dilaton $\Phi$ is always quadratic in the radial coordinate, i.e., $\Phi(z)=\phi_{\infty} z^{2}$. It turns out that spontaneous chiral symmetry breaking can be realized as long as we impose the following UV asymptotic behavior [19]:

$$
a_{I}(\Phi \rightarrow 0)=-a_{0} \Phi
$$

Having fixed the asymptotic form of the function $a(\Phi)$, one may build an interpolating function considering these asymptotic constraints. We consider first the following simple interpolation ${ }^{1}$ :

models of type I:

$$
a_{\mathrm{I}}(\Phi)=\Phi\left[\frac{-a_{0}^{2}+\Phi^{2}}{a_{0}+\Phi^{2}}\right]
$$

However, as long as the coupling $a(\Phi)$ becomes minimal in the IR, the asymptotic form in the UV should not be necessarily negative. As we will see, it will be sufficient to impose that $a(\Phi)$ is negative for intermediate values of $\Phi$

\footnotetext{
${ }^{1}$ The choice of this function was motivated by the interpolation presented in Ref. [19]; in our notation, it would take the form $a(\Phi)=\Phi\left[-a_{0}+\left(1+a_{0}\right) \tanh \left(\frac{\Phi}{a_{0}}\right)\right]$. Note that the interpolation using powers of the dilaton field is smoother than the interpolation using hyperbolic function.
} 

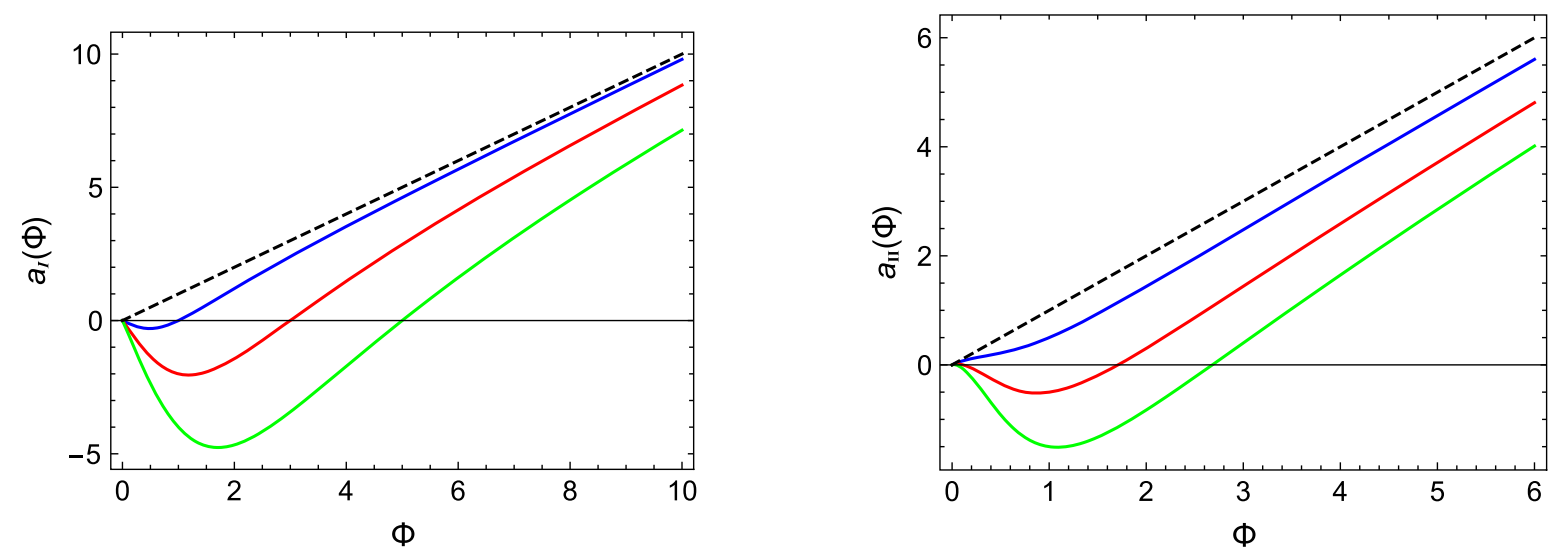

FIG. 1. The nonminimal dilaton couplings $a_{\mathrm{I}}(\Phi)$ (left panel) and $a_{\mathrm{II}}(\Phi)$ (right panel) for the selected values $a_{0}=1$ (blue), $a_{0}=3$ (red), and $a_{0}=5$ (green). The black dashed lines in both panels correspond to the limit $a_{0} \rightarrow 0$ where the dilaton couplings reduce to the minimal form $a(\Phi)=\Phi$.

(or equivalently $z$ ). This choice will provide improved results compared to models of type I. Its asymptotic behavior in the UV is such that

$$
a_{\mathrm{II}}(\Phi \rightarrow 0)=\Phi
$$

Considering this case, we build the following interpolation function:

models of type II:

$$
a_{\mathrm{II}}(\Phi)=\Phi-\frac{a_{0} \Phi^{3 / 2}}{1+\Phi^{2}}
$$

The nonminimal dilaton couplings (17) and (19) are displayed in Fig. 1 for selected values of $a_{0}$. The nonminimal couplings can be thought of as deformations of the original soft wall model characterized by a new free parameter $a_{0}$; we will show later that we need this additional parameter to induce a transition between explicit and spontaneous chiral symmetry breaking.

As regards the dilaton coupling $b(\Phi)$ to the 5D gauge fields, we will consider the following possibilities:

models of type A:

$$
b(\Phi)=a(\Phi)
$$

That is, in models of type A, the gauge fields couple nonminimally to the dilaton in exactly the same way as the tachyonic field;

TABLE I. Nonminimal couplings $a(\Phi)$ and $b(\Phi)$ in models of type IA, IB, IIA, and IIB.

\begin{tabular}{lcccc}
\hline \hline Model & IA & IB & IIA & IIB \\
\hline Coupling $a(\Phi)$ & $a_{\mathrm{I}}(\Phi)$ & $a_{\mathrm{I}}(\Phi)$ & $a_{\mathrm{II}}(\Phi)$ & $a_{\mathrm{II}}(\Phi)$ \\
Coupling $b(\Phi)$ & $a_{\mathrm{I}}(\Phi)$ & $\Phi$ & $a_{\mathrm{II}}(\Phi)$ & $\Phi$ \\
\hline \hline
\end{tabular}

models of type B:

$$
b(\Phi)=\Phi
$$

In this case, only the tachyonic field couples nonminimally to the dilaton.

Table I summarizes the four different models considered in this work.

At this point, it is important to make some remarks regarding the nonminimal couplings in models of type A and type B. First, one should note that the nonminimal couplings in models IA and IIA are indeed consistent with a D-brane construction, in which one adds space-time filling D-branes in the 5D bulk theory. ${ }^{2}$ In those models, the nonminimal couplings can be redefined in terms of an effective dilaton field in a such way that it fixes the coupling to it as an overall $e^{-\tilde{\Phi}}$ in (5), where the effective dilaton field, $\tilde{\Phi}$, now has a nontrivial radial profile in the UV and IR regimes, in contrast with the pure quadratic behavior of the original soft wall [10].

On the other hand, the nonminimal couplings in model IB and IIB employed here seem to be inconsistent with a D-brane construction in the sense that they do not come from perturbative string theory. This is because the gauge fields and the tachyon field couple to the dilaton in different ways, i.e., $b(\Phi) \neq a(\Phi)$. Nevertheless, from the point of view of an effective holographic model, such nonminimal couplings are widely used in the context of Einstein-Maxwell-Dilaton holography in AdS/CMT (condensed matter theory) (for a review, see Ref. [57]) and holographic QCD at finite temperature and density [30,58-61]. Nonminimal dilaton couplings to tachyon and gauge fields were considered in the holographic approach to QCD in the Veneziano limit [48,62]. However, beyond tree-level approximation in string

\footnotetext{
${ }^{2}$ For a more detailed discussion on how this construction works in AdS/CFT, we refer the reader to Refs. $[37,45,56]$.
} 
theory, one should be careful since there are higher-order $\alpha^{\prime}$ corrections that could affect the nonminimal couplings in such way that it might render the theory inconsistent somehow. Currently, it is not clear if those nonminimal couplings in effective holographic models can be embedded in a well-established string theory $[29,57,63]$.

\section{Violation of the BF bound}

In this subsection, we investigate the violation of the Breitenlohner-Freedman (BF) lower bound $m_{B F}^{2}=-4$ [64] for the 5D effective mass of a scalar perturbation $S(x, z)$ around the trivial solution $v(z)=0$. This scalar perturbation satisfies the linear differential equation

$$
\left[\partial_{z}+3 A_{s}^{\prime}-a^{\prime}\right] \partial_{z} S+\square S-e^{2 A_{s}} m_{X}^{2} S=0 .
$$

Redefining the scalar field as

$$
S=e^{\frac{a}{2}} \bar{S},
$$

the tachyonic differential equation takes the $\mathrm{AdS}_{5}$ form

$$
\left[\partial_{z}+3 A_{s}^{\prime}\right] \partial_{z} \bar{S}+\square \bar{S}-e^{2 A_{s}} \bar{m}_{X}^{2}(z) \bar{S}=0,
$$

where

$$
\bar{m}_{X}^{2}(z)=m_{X}^{2}-e^{-2 A_{s}}\left[\frac{a^{\prime \prime}}{2}+\frac{a^{\prime}}{2}\left(3 A_{s}^{\prime}-\frac{a^{\prime}}{2}\right)\right]
$$

is an effective 5D running mass. Since $A_{s}=-\ln z$ is the AdS warp factor, the evolution of the effective 5D mass $\bar{m}_{X}^{2}$ with the radial direction $z$ may imply the violation of the $\mathrm{BF}$ found for the scalar field $S(x, z)$; namely, there maybe some regions in $z$ where $\bar{m}_{X}^{2}(z)<-4$. The violation of the $\mathrm{BF}$ found indicates the instability of the trivial solution

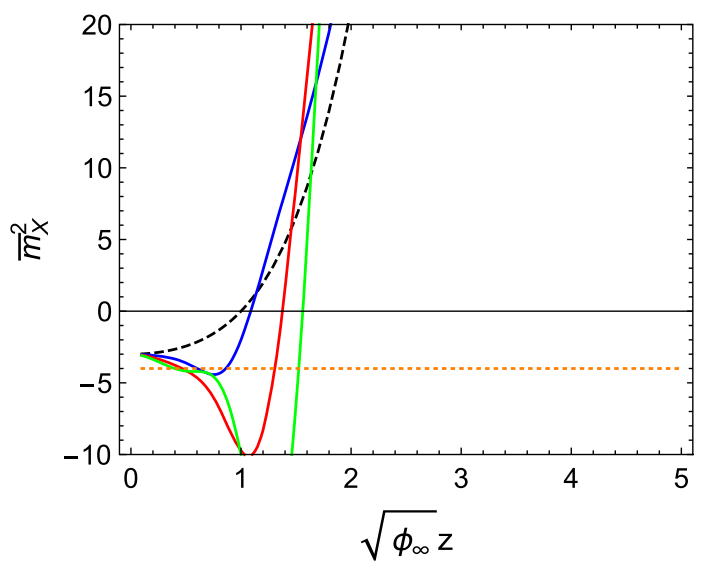

$v(z)=0$ which is the gravity dual of a chirally symmetric vacuum. This is what we expect if we want to describe spontaneous chiral symmetry breaking because if we consider the limit of massless quarks (chiral limit) the instability of the trivial solution $v(z)=0$ indicates the presence of a nontrivial solution $v(z) \neq 0$ that breaks the chiral symmetry spontaneously. In Fig. 2, we plot the 5D effective mass $\bar{m}_{X}^{2}$ as a function of the dimensionless radial coordinate $\sqrt{\phi_{\infty}} z$. The violation of the $\mathrm{BF}$ bound at intermediate values of $z$ indicates the instability of the trivial vacuum.

In the next subsection, we solve the nonlinear differential equation (14) for the tachyonic field $v(z)$ and find a nontrivial solution that is consistent with the spontaneous breaking of chiral symmetry in the chiral limit.

\section{Background solution for the tachyonic field}

Once the nonminimal coupling was fixed, one may solve the differential equation (14) in the asymptotic regions. Below, we describe this procedure for the UV region (near the boundary) and the IR region (far from the boundary).

\section{UV asymptotics}

In the UV, or close to the boundary, we consider the power ansatz for the rescaled tachyon field $\tilde{v}(z)=\sqrt{\lambda} v(z)$. The asymptotic solution to (14) takes the form

$\tilde{v}=\tilde{c}_{1} z+\tilde{d}_{3} z^{3} \ln z+\tilde{c}_{3} z^{3}+\tilde{c}_{4} z^{4}+\tilde{d}_{5} z^{5} \ln z+\tilde{c}_{5} z^{5}+\cdots$

It is worth mentioning that the parameter $\tilde{c}_{1}$ is related to the quark mass $m_{q}$ and chiral condensate $\Sigma=\langle\bar{q} q\rangle$ in the dual field theory through the relations $\tilde{c}_{1}=\zeta \tilde{m}_{q}$ and $\tilde{c}_{3}=$ $\tilde{\Sigma} /(2 \zeta)$ [51], where we have defined $\tilde{m}_{q}=\sqrt{\lambda} m_{q}$ and $\tilde{\Sigma}=\sqrt{\lambda} \Sigma$. The normalization constant $\zeta$ is fixed as

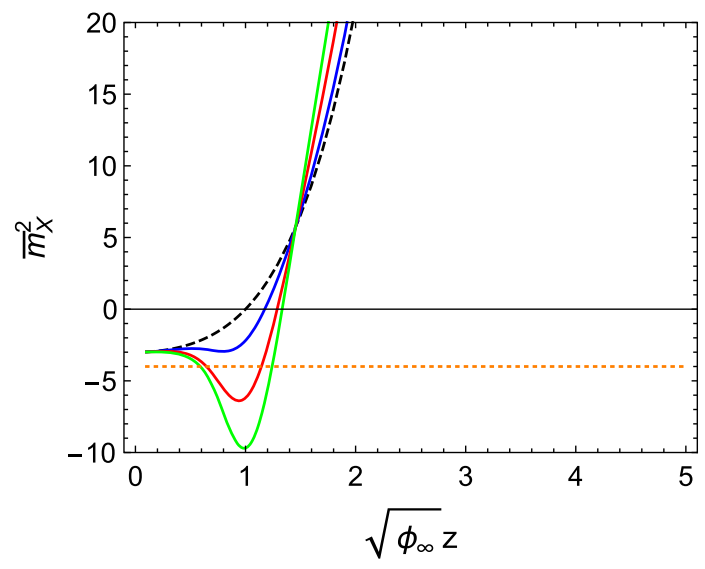

FIG. 2. The evolution of the 5D effective mass $\bar{m}_{X}^{2}$ with the dimensionless radial coordinate $\sqrt{\phi_{\infty}} z$ for models of type I (left panel) and models of type II (right panel). In both panels, the blue, red, and green solid lines correspond to $a_{0}=1, a_{0}=3$, and $a_{0}=5$ respectively. The black dashed line in both panels correspond to the limit $a_{0} \rightarrow 0$ where the dilaton couplings become minimal (original soft wall model). The horizontal line (orange dotted) represents the BF bound $m_{\mathrm{BF}}^{2}=-4$. 
$\zeta=\sqrt{N_{c}} /(2 \pi)$ by large $N_{c}$ counting rules for the scalar correlation function [65]. For models of type I, the coefficients $\tilde{d}_{3}, \tilde{c}_{4}, \cdots$ in Eq. (26) are given by

$\tilde{d}_{3}=\frac{\tilde{c}_{1} \phi_{\infty}}{4}\left(\frac{\tilde{c}_{1}^{2}}{\phi_{\infty}}-4 a_{0}\right), \quad \tilde{c}_{4}=0$,

$\tilde{d}_{5}=\frac{3 \tilde{c}_{1} \phi_{\infty}^{2}}{64}\left(\frac{\tilde{c}_{1}^{2}}{\phi_{\infty}}-4 a_{0}\right)^{2}$,

$\tilde{c}_{5}=\frac{\tilde{c}_{1} \phi_{\infty}^{2}}{256}\left(\frac{\tilde{c}_{1}^{2}}{\phi_{\infty}}-4 a_{0}\right)\left(\frac{48 \tilde{c}_{3}}{\tilde{c}_{1} \phi_{\infty}}-\frac{9 \tilde{c}_{1}^{3}}{\tilde{c}_{1} \phi_{\infty}}+20 a_{0}\right)$,

while for models of type II, they are

$$
\begin{aligned}
& \tilde{d}_{3}=\frac{\tilde{c}_{1} \phi_{\infty}}{4}\left(\frac{\tilde{c}_{1}^{2}}{\phi_{\infty}}+4\right), \quad \tilde{c}_{4}=-a_{0} \tilde{c}_{1} \phi_{\infty}^{3 / 2}, \\
& \tilde{d}_{5}=\frac{3 \tilde{c}_{1} \phi_{\infty}^{2}}{64}\left(\frac{\tilde{c}_{1}^{2}}{\phi_{\infty}}+4\right)^{2}, \\
& \tilde{c}_{5}=\frac{\tilde{c}_{1} \phi_{\infty}^{2}}{256}\left(\frac{\tilde{c}_{1}^{2}}{\phi_{\infty}}+4\right)\left(\frac{48 \tilde{c}_{3}}{\tilde{c}_{1} \phi_{\infty}}-\frac{9 \tilde{c}_{1}^{2}}{\phi_{\infty}}-20\right) .
\end{aligned}
$$

\section{IR asymptotics}

In the IR region, there are two independent solutions; see Eq. (13). In order to avoid singularities in the action (5), we choose the regular solution. The subleading terms may be obtained considering the power ansatz

$$
\tilde{v}=\tilde{C}_{0}+\frac{\tilde{C}_{2}}{z^{2}}+\frac{\tilde{C}_{4}}{z^{4}}+\frac{\tilde{C}_{5}}{z^{5}}+\cdots
$$

Thus, plugging them into the differential equation (14) and solving order by order, we get the coefficients for models of type I,

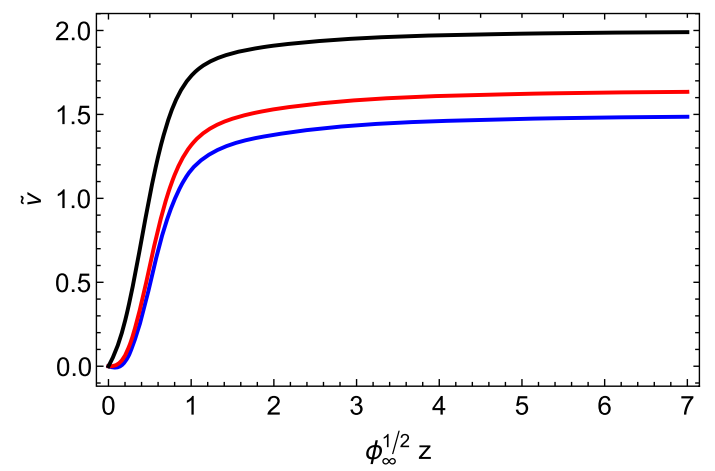

$$
\begin{aligned}
& \tilde{C}_{2}=\frac{\tilde{C}_{0}}{8 \phi_{\infty}}\left(\tilde{C}_{0}^{2}-6\right), \\
& \tilde{C}_{4}=\frac{3 \tilde{C}_{0}}{128 \phi_{\infty}^{2}}\left(\tilde{C}_{0}^{2}-10\right)\left(\tilde{C}_{0}^{2}-6\right), \\
& \tilde{C}_{5}=0 .
\end{aligned}
$$

While the coefficients for models of type II are

$$
\begin{aligned}
& \tilde{C}_{2}=\frac{\tilde{C}_{0}}{8 \phi_{\infty}}\left(\tilde{C}_{0}^{2}-6\right), \\
& \tilde{C}_{4}=\frac{3 \tilde{C}_{0}}{128 \phi_{\infty}^{2}}\left(\tilde{C}_{0}^{2}-10\right)\left(\tilde{C}_{0}^{2}-6\right), \\
& \tilde{C}_{5}=\frac{a_{0} \tilde{C}_{0}}{40 \phi_{\infty}^{5 / 2}}\left(\tilde{C}_{0}^{2}-6\right) .
\end{aligned}
$$

It is worth pointing out that the contribution of the parameter $a_{0}$ is relevant in the UV regime, while in the IR, it contributes in terms greater than $\tilde{C}_{4}$. Note also that the leading term in the IR is constant, while it becomes an exact solution when $\tilde{C}_{0}=6$, which coincides with the minimum of the tachyon potential.

\section{Numerical results}

In the sequence, we present and discuss the numerical results obtained solving the differential equation (14). To solve the problem numerically, we use as "initial condition" the asymptotic solution obtained in the IR. Then, we integrate numerically from the IR to the UV varying the independent parameter $\tilde{C}_{0}$ once $a_{0}$ was fixed. Finally, we read off the values for $\tilde{c}_{1}$ and $\tilde{c}_{3}$ by matching the numerical solution against the asymptotic solution in the UV. To finish this section, we plot the tachyon profile for selected values of the parameter $\tilde{C}_{0}$. In the left panel of Fig. 3, we display the tachyon obtained in models of type I for $\tilde{C}_{0}=1.5$ (blue), $\tilde{C}_{0}=1.648$ (red), and $\tilde{C}_{0}=2.0$ (black). In the right panel of this figure, we display the tachyon obtained in models of type II for selected values of $\tilde{C}_{0}$

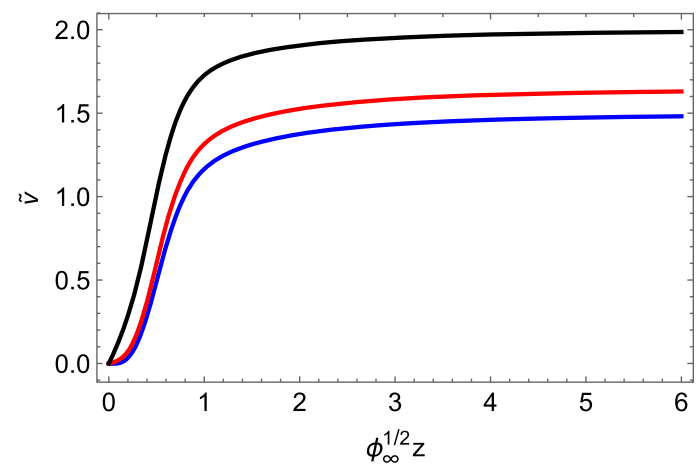

FIG. 3. Left: the tachyon $\tilde{v}$ as a function of $\phi_{\infty}^{1 / 2} z$ for models of type I. Right: the tachyon $\tilde{v}$ as a function of $\phi_{\infty}^{1 / 2} z$ for models of type II. 

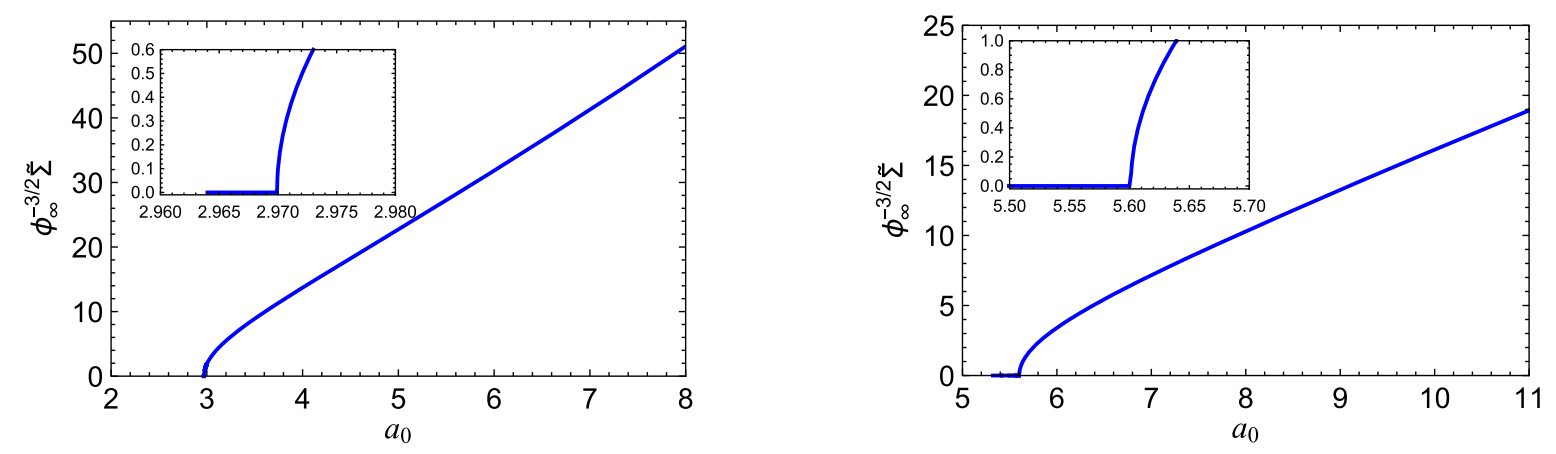

FIG. 4. Left: the dimensionless condensate $\phi_{\infty}^{-3 / 2} \tilde{\Sigma}$ as a function of $a_{0}$ in the chiral limit, i.e., $c_{1}=0$, for models of type I. Right: the dimensionless condensate $\phi_{\infty}^{-3 / 2} \tilde{\Sigma}$ as a function of $a_{0}$ in the chiral limit for models of type II.

\section{E. Spontaneous chiral symmetry breaking/chiral limit}

Here, we investigate the limit of massless quarks, which is reached in the limit of zero $\tilde{c}_{1}$ since $\tilde{c}_{1} \propto \tilde{m}_{q}$, where $\tilde{m}_{q}$ is the rescaled quark mass. In the left panel of Fig. 4 , we display the numerical results of $\phi_{\infty}^{-3 / 2} \tilde{c}_{3}$ as a function of the parameter $a_{0}$ obtained in models of type I. As can be seen, there is a critical value at $a_{0}=a_{0_{c}} \approx 2.970$ where $\phi_{\infty}^{-3 / 2} \tilde{c}_{3}$ starts to increase; see the inset for details. This figure is showing us that there is a nonzero condensate, $\phi_{\infty}^{-3 / 2} \tilde{c}_{3} \neq 0$, for $a_{0} \geq a_{0_{c}}$. It is also interesting to point out that the condensate is zero in the region $a_{0}<a_{0_{c}}$, where the symmetry breaking is explicit. Therefore, there is a transition between explicit and spontaneous symmetry breaking at $a_{0}^{c}$. In turn, the right panel of Fig. 4 shows the same results for models of type II. As can be seen, for this model, we have $a_{0}^{c} \approx 5.60$.

Furthermore, we display the corresponding results of the dimensionless chiral condensate $\phi_{\infty}^{-3 / 2} \tilde{\Sigma}$ as a function of $\tilde{C}_{0}^{\min }$ in the left panel of Fig. 5 with a solid blue line for models of type I and a solid red line for models of type II. Note that $\tilde{C}_{0}^{\min }$ corresponds to each $\phi_{\infty}^{-3 / 2} \tilde{\Sigma}$ calculated in the chiral limit; this means that each $\tilde{C}_{0}^{\min }$ corresponds to each

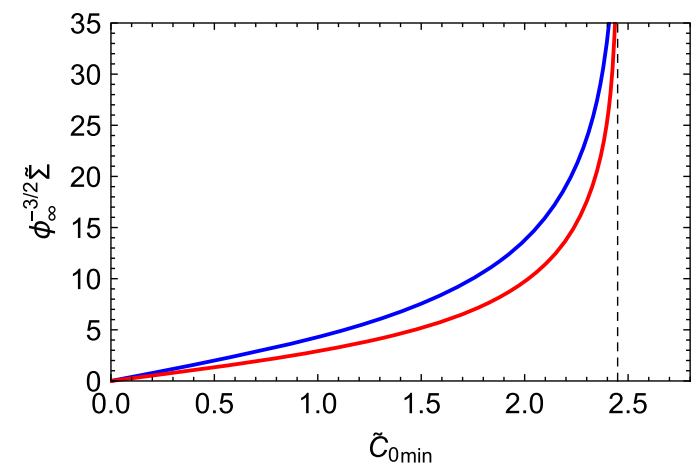

$a_{0}$, as shown in the right panel of this figure. As can be seen, the value of $\phi_{\infty}^{-3 / 2} \tilde{\Sigma}$ goes to zero when $\tilde{C}_{0}^{\text {min }}$ goes to zero. However, it diverges when $\tilde{C}_{0}^{\min }$ approaches to $\tilde{C}_{0}^{\max }=\sqrt{6}$ represented by vertical dashed line. In turn, in the right panel of this figure, we display the results of $\tilde{C}_{0}^{\min }$ as a function of $a_{0}$ for both models I and II. In this figure, we may identify the physical region where $\tilde{c}_{1}>0$. Therefore, fixing the value of $a_{0}$, the physical region belongs to the interval $\tilde{C}_{0}^{\min }<\tilde{C}_{0}<\sqrt{6}$. It is also worth mentioning that for values of $a_{0}$ in the interval $a_{0} \leq 6.954$, one can go continuously from one minimum of the tachyon potential, $-\sqrt{6}$, to the other, $\sqrt{6}$, passing through the trivial solution. However, for values larger than $a_{0} \approx 6.954, \tilde{C}_{0}$ is constrained to take values close to the minimum of the tachyon potential and the trivial solution. We explain details on the numerical results in Appendix A.

Finally, in order to correctly describe spontaneous symmetry breaking, these results must be consistent with the emergence of pseudo-Nambu-Goldstone bosons in the spectrum. We will calculate the spectrum in the following section and check if these states are present in the holographic model.

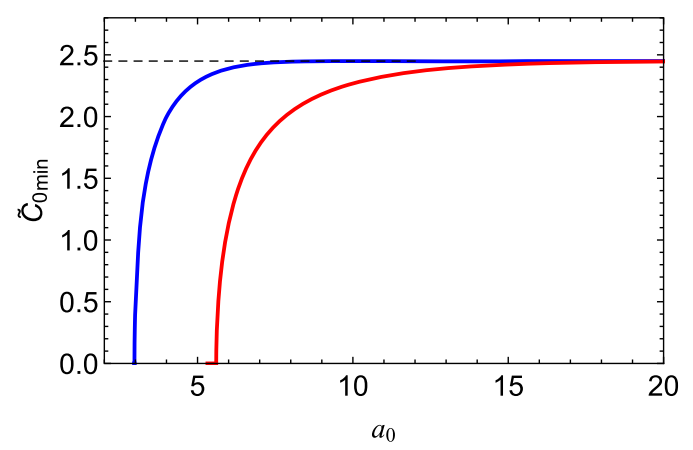

FIG. 5. Left: the dimensionless condensate $\phi_{\infty}^{-3 / 2} \tilde{\Sigma}$ as a function of $\tilde{C}_{0}^{\min }$ in the chiral limit; the blue solid lines are the results for models of type I, while red solid lines for models of type II. Right: $\tilde{C}_{0}^{\min }$ as a function of $a_{0}$. In both figures, dashed lines represent the minimum of the Higgs potential $\tilde{C}_{0}^{\max }=\sqrt{6}$. 


\section{F. Chiral condensate in the physical region}

Here, we show results for the rescaled chiral condensate $\tilde{\Sigma}$ as a function of the rescaled quark mass $\tilde{m}_{q}$, obtained from the relations $\tilde{c}_{3}=\tilde{\Sigma} /(2 \zeta)$ and $\tilde{c}_{1}=\tilde{m}_{q}$, in units of $\phi_{\infty}$ in the physical region $\tilde{m}_{q}>0$. Our numerical results for models of type I are displayed in the left panel of Fig. 6, where the blue line represents the results for $a_{0}=2.8$ and the red line represents the results for $a_{0}=3.5$. As can be seen, the chiral condensate is nonzero in the chiral limit for $a_{0}>a_{0}^{c} \approx 2.970$ (red line). It is worth pointing out that the chiral condensate becomes negative in the intermediate region. In turn, our numerical results for models of type II are displayed in the right panel of Fig. 6. As can be seen, the chiral condensate becomes a monotonic increasing function of the quark mass. Analogous to models of type I, we have nonzero chiral condensate in the chiral limit for $a_{0}>$ $a_{0}^{c} \approx 5.60$ (red line).

For a detailed discussion of the numerical procedure and to see the symmetric behavior of the tachyon, see Appendix A.

\section{G. Fixing the model parameters}

To finish this section, we are going to fix the parameters of the model. The dilaton parameter $\phi_{\infty}$ is fixed to $\phi_{\infty}=(0.388 \mathrm{GeV})^{2}$, as in the soft wall model [66]. This leads to a good description of the meson spectrum in the vectorial sector. As described in Sec. III E, models of type I (type II) lead to spontaneous chiral symmetry breaking when $a_{0} \geq 2.97\left(a_{0} \geq 5.6\right)$. For models of type I (type II), we choose $a_{0}=3.5\left(a_{0}=6.5\right)$, corresponding to $\phi_{\infty}^{-3 / 2} \tilde{\Sigma} /(2 \zeta)=8.8988\left(\phi_{\infty}^{-3 / 2} \tilde{\Sigma} /(2 \zeta)=5.4367\right)$; see Fig. 4. In the next section, we will see that these parameter choices allow us to obtain good results for the meson spectrum in the scalar sector.

Considering the relation between the parameter $\tilde{c}_{3}$ and the rescaled chiral condensate, $\tilde{c}_{3}=\tilde{\Sigma} /(2 \zeta)$ [51], where $\zeta=$ $\sqrt{N_{c}} /(2 \pi)$ [65], and recalling the definition $\tilde{\Sigma}=\sqrt{\lambda}\langle\bar{q} q\rangle$, we obtain the relation

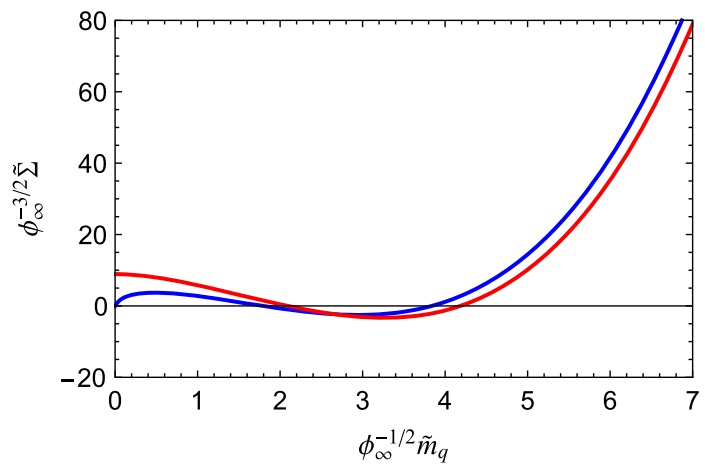

TABLE II. Parameters in the four different models fixed in order to reproduce the meson spectrum.

\begin{tabular}{lcccc}
\hline \hline Parameter & Model IA & Model IB & Model IIA & Model IIB \\
\hline$a_{0}$ & 3.5 & 3.5 & 6.5 & 6.5 \\
$\phi_{\infty}$ & $(0.388 \mathrm{GeV})^{2}$ & $(0.388 \mathrm{GeV})^{2}$ & $(0.388 \mathrm{GeV})^{2}$ & $(0.388 \mathrm{GeV})^{2}$ \\
$\lambda$ & 160 & 380 & 60 & 413 \\
\hline \hline
\end{tabular}

$$
\phi_{\infty}^{-3 / 2} \frac{\sqrt{\lambda}\langle\bar{q} q\rangle}{2 \zeta}=\phi_{\infty}^{-3 / 2} \tilde{c}_{3}
$$

For fixed $\phi_{\infty}$ and $a_{0}$, different choices of the parameter $\lambda$ lead to different values for the chiral condensate $\langle\bar{q} q\rangle$. We fix the parameter $\lambda$ separately for each type of model in order to obtain good results for the meson spectrum in the axialvector and pseudoscalar sector; namely, in order to avoid crossings between the fundamental and excited states in the axial and pseudoscalar sector, $\lambda$ has to be above some critical value that depends of each model type.

For the models of type IA, IB, IIA, and IIB, we choose $\lambda=160, \lambda=380, \lambda=60$, and $\lambda=413$, respectively. These choices of parameters lead to $\langle\bar{q} q\rangle=(0.245 \mathrm{GeV})^{3}$ for model IA, $\langle\bar{q} q\rangle=(0.283 \mathrm{GeV})^{3}$ for models IB and IIA, and $\langle\bar{q} q\rangle=(0.205 \mathrm{GeV})^{3}$ for model IIB. These results are of the same order as those obtained in lattice QCD; see, for instance, Refs. [67-70].

Table II summarizes our choice of parameters for the four different type of models. Finally, the only remaining parameter in our models is the (current) quark mass $m_{q}$. In the next section, we will initially treat $m_{q}$ as a free parameter so that we can investigate the evolution from the chiral limit $m_{q}=0$ to the heavy quark regime (large $m_{q}$ ). For the scalar, axial-vector and pseudoscalar mesons, it will be necessary to fix $m_{q}$ close to the physical quark mass in order to compare our results for the masses and decay constants against experimental data and other results available in the literature.

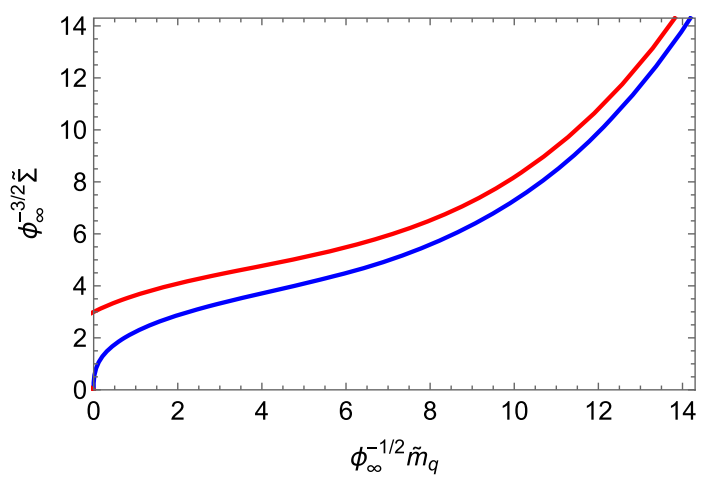

FIG. 6. Left: the dimensionless condensate $\phi_{\infty}^{-3 / 2} \tilde{\Sigma}$ as a function of the dimensionless quark mass $\phi_{\infty}^{-1 / 2} \tilde{m}_{q}$ for models of type I; blue lines are the results for $a_{0}=2.8$, while red lines are the results for $a_{0}=3.5$. Right: the dimensionless condensate $\phi_{\infty}^{-3 / 2} \tilde{\Sigma}$ as a function of the dimensionless quark mass $\phi_{\infty}^{-1 / 2} \tilde{m}_{q}$ for models of type II; blue lines are the results for $a_{0}=5.6$, while red lines are the results for $a_{0}=6.5$. 


\section{MESON SPECTRUM}

In this section, we calculate the masses of mesons in the vectorial, scalar, axial, and pseudoscalar sector. First, we obtain the 5D differential equations for the tachyonic and gauge field perturbations. Decomposing these perturbations into irreducible representations of the Lorentz group, we identify the normalizable solutions with the wave functions representing mesons in the four-dimensional (4D) field theory. In the vectorial, scalar, and axial sector, we recast the differential equations in a Schrödinger form and find the squared masses as the energy eigenvalues in the Schrödinger problem. For the pseudoscalar mesons, the spectrum will be obtained by solving directly the coupled differential equations satisfied by the normalizable modes.

\section{A. Expanding the action}

Since the background gauge fields are zero, we can expand trivially around it and consider the fluctuations $A_{m}^{(L / R)}$. It is convenient to rewrite those fluctuations in terms of vectorial and axial gauge fields

$$
A_{m}^{(L / R)}=V_{m} \pm A_{m}
$$

On the other hand, the tachyon field has a nontrivial background solution, and we can expand around it as

$$
2 X=e^{2 i \pi}(v+S),
$$

where $\pi=\pi^{c} T^{c}$ and $S$ is a real scalar field. Note that the fields $\pi^{c}$ transform as a triplet in the adjoint representation of $S U(2)$. They will give rise to a description of 4D pions, whereas the field $S$ will correspond to scalar mesons. We expand the action (5) using (33) and (34). The expansion can be written as

$$
S=S_{0}+S_{1}+S_{2}+\ldots
$$

where

$$
\begin{aligned}
S_{0}= & -\int d^{4} x \int d z e^{3 A_{s}-a}\left[\frac{1}{2}\left(\partial_{z} v\right)^{2}+e^{2 A_{s}} U(v)\right], \\
S_{1}= & -\int d^{4} x \int d z e^{3 A_{s}-a}\left[\left(\partial_{z} v\right)\left(\partial_{z} S\right)+e^{2 A_{s}} \frac{d U}{d v} S\right], \\
S_{2}= & -\int d^{4} x \int d z\left\{e^{3 A_{s}-a}\left[\frac{1}{2}\left(\partial_{\hat{m}} S\right)^{2}+\frac{1}{2} e^{2 A_{s}} \frac{d^{2} U}{d v^{2}} S^{2}\right]\right. \\
& \left.+\frac{1}{g_{5}^{2}} e^{A_{s}-b}\left[\frac{1}{4} v_{\hat{m} \hat{n}}^{c}{ }^{2}+\frac{1}{4} a_{\hat{m} \hat{n}}^{c}+\frac{\beta}{2}\left(\partial_{\hat{m}} \pi^{c}-A_{\hat{m}}^{c}\right)^{2}\right]\right\} .
\end{aligned}
$$

where

$$
\beta \equiv e^{2 A_{s}+b-a} g_{5}^{2} v^{2}
$$

and $v_{\hat{m} \hat{n}}^{c}=\partial_{\hat{m}} V_{\hat{n}}^{c}-\partial_{\hat{n}} V_{\hat{m}}^{c}$ (similarly for $a_{\hat{m} \hat{n}}^{c}$ ). The indices $(\hat{m}, \hat{n})$ correspond to coordinates in the 5D flat metric $\eta_{\hat{m} \hat{n}}$, and the index $c=(1,2,3)$ corresponds to the adjoint representation of the $S U(2)$ isospin group. The actions $S_{0}$ and $S_{1}$ become surface terms after replacing the tachyon field equation in (12).

The action $S_{2}$ is responsible for the dynamics of the field fluctuations $S, V^{\hat{m}, c}, A^{\hat{m}, c}$, and $\pi^{c}$. The variation of $S_{2}$ can be written as

$$
\delta S_{2}=\delta S_{2}^{\mathrm{Bulk}}+\delta S_{2}^{\mathrm{Bdy}} .
$$

The bulk term $\delta S_{2}^{\text {Bulk }}$ can be written as

$$
\begin{aligned}
\delta S_{2}^{\text {Bulk }}= & \int d^{4} x \int d z\left\{e^{3 A_{s}-a} \delta S\left[e^{a-3 A_{s}} \partial_{\hat{m}}\left(e^{3 A_{s}-a} \partial^{\hat{m}} S\right)-e^{2 A_{s}} \frac{d^{2} U}{d v^{2}} S\right]+\frac{1}{g_{5}^{2}} \delta V_{\hat{n}}^{c} \partial_{\hat{m}}\left(e^{A_{s}-b} v_{c}^{\hat{m} \hat{n}}\right)\right. \\
& \left.+\frac{1}{g_{5}^{2}} e^{A_{s}-b} \delta A_{\hat{n}}^{c}\left[e^{b-A_{s}} \partial_{\hat{m}}\left(e^{A_{s}-b} a_{c}^{\hat{m} \hat{n}}\right)+\beta\left(\partial^{\hat{m}} \pi^{c}-A^{\hat{m}, c}\right)\right]+\frac{1}{g_{5}^{2}} \delta \pi^{c} \partial_{\hat{m}}\left[e^{A_{s}-b} \beta\left(\partial^{\hat{m}} \pi^{c}-A^{\hat{m}, c}\right)\right]\right\} .
\end{aligned}
$$

The boundary term $\delta S_{2}^{\mathrm{Bdy}}$ can be written as

$$
\begin{aligned}
\delta S_{2}^{\text {Bdy }}= & -\int d^{4} x \int d z \partial_{\hat{m}}\left\{e^{3 A_{s}-a}\left(\partial^{\hat{m}} S\right) \delta S\right. \\
& +\frac{1}{g_{5}^{2}} e^{A_{s}-b} v_{c}^{\hat{m} \hat{n}} \delta V_{\hat{n}}^{c}+\frac{1}{g_{5}^{2}} e^{A_{s}-b} a_{c}^{\hat{m} \hat{n}} \delta A_{\hat{n}}^{c} \\
& \left.+\frac{1}{g_{5}^{2}} \beta e^{A_{s}-b}\left(\partial^{\hat{m}} \pi^{c}-A^{\hat{m}, c}\right) \delta \pi^{c}\right\} .
\end{aligned}
$$

Imposing periodic boundary conditions in the $x^{\mu}$ coordinates, we end up with a boundary term in the $z$ coordinate,

$$
\begin{aligned}
\delta S_{2}^{\text {Bdy }}= & -\int d^{4} x\left\{e^{3 A_{s}-a}\left(\partial_{z} S\right) \delta S\right. \\
& +\frac{1}{g_{5}^{2}} e^{A_{s}-b} v_{c}^{\hat{z} \hat{\mu}} \delta V_{\hat{\mu}}^{c}+\frac{1}{g_{5}^{2}} e^{A_{s}-b} a_{c}^{\hat{z} \hat{\mu}} \delta A_{\hat{\mu}}^{c} \\
& \left.+\frac{1}{g_{5}^{2}} \beta e^{A_{s}-b}\left(\partial^{\hat{z}} \pi^{c}-A^{\hat{z}, c}\right) \delta \pi^{c}\right\}_{z=\epsilon}^{z \rightarrow \infty}
\end{aligned}
$$

The quadratic behavior $\Phi(z) \sim z^{2}$ at large $z$ implies that the terms with couplings $\exp (-a)$ and $\exp (-b)$ vanish exponentially in the limit $z \rightarrow \infty$. Imposing also Dirichlet 
boundary conditions at $z=\epsilon$, one guarantees the vanishing of (41).

The vanishing of $\delta S_{2}$ then implies the condition $\delta S_{2}^{\text {Bulk }}=0$ for the bulk term. This leads to the field equations

$$
\begin{aligned}
e^{a-3 A_{s}} \partial_{\hat{m}}\left(e^{3 A_{s}-a} \partial^{\hat{m}} S\right)-e^{2 A_{s}} \frac{d^{2} U}{d v^{2}} S & =0, \\
\partial_{\hat{m}}\left[e^{A_{s}-b} \beta\left(\partial^{\hat{m}} \pi^{a}-A^{\hat{m}, a}\right)\right] & =0, \\
\partial_{\hat{m}}\left(e^{A_{s}-b} v_{a}^{\hat{m} \hat{n}}\right) & =0, \\
e^{b-A_{s}} \partial_{\hat{m}}\left(e^{A_{s}-b} a_{a}^{\hat{m} \hat{n}}\right)+\beta\left(\partial^{\hat{n}} \pi-A^{a, \hat{n}}\right) & =0 .
\end{aligned}
$$

Note that the action and field equations are invariant under the vectorial gauge symmetry

$$
V^{\hat{m}, c} \rightarrow V^{\hat{m}, c}-\partial^{\hat{m}} \lambda_{V}^{c}
$$

and the (residual) axial gauge symmetry

$$
A^{\hat{m}, c} \rightarrow A^{\hat{m}, c}-\partial^{\hat{m}} \lambda_{A}^{c}, \quad \pi^{c} \rightarrow \pi^{c}-\lambda_{A}^{c} .
$$

The gauge fields can be decomposed as $V_{\hat{m}}=\left(V_{z}, V_{\hat{\mu}}\right)$, $A_{\hat{m}}=\left(A_{z}, A_{\hat{\mu}}\right)$ and also the derivatives $\partial_{\hat{m}}=\left(\partial_{z}, \partial_{\hat{\mu}}\right)$. The vectorial gauge symmetry in (43) allows us to set $V_{z}=0$, while the residual axial gauge symmetry in (44) can be used to set $A_{z}=0$. The $4 \mathrm{D}$ vectors $V_{\hat{\mu}}$ and $A_{\hat{\mu}}$ admit also the Lorentz decomposition

$$
V_{\hat{\mu}, c}=V_{\hat{\mu}, c}^{\perp}+\partial_{\hat{\mu}} \xi^{c}, \quad A_{\hat{\mu}, c}=A_{\hat{\mu}, c}^{\perp}+\partial_{\hat{\mu}} \varphi^{c} .
$$

The scalar fields $\xi^{c}$ are not dynamical and can be set to zero. Using these results, the 5D equations (42) become the set of independent field equations

$$
\begin{aligned}
& {\left[\partial_{z}+3 A_{s}^{\prime}-a^{\prime}\right] \partial_{z} S+\square S-e^{2 A_{s}} \frac{d^{2} U}{d v^{2}} S=0 \quad \text { (scalar sector), }} \\
& {\left[\partial_{z}+A_{s}^{\prime}-b^{\prime}\right] \partial_{z} V_{\perp}^{\hat{\mu}, c}+\square V_{\perp}^{\hat{\mu}, c}=0 \quad \text { (vectorial sector) },} \\
& {\left[\partial_{z}+A_{s}^{\prime}-b^{\prime}\right] \partial_{z} A_{\perp}^{\hat{\mu}, c}+\square A_{\perp}^{\hat{\mu}, c}-\beta A_{\perp}^{\hat{\mu}, c}=0 \quad \text { (axial sector) }} \\
& {\left[\partial_{z}+A_{s}^{\prime}-b^{\prime}\right] \partial_{z} \varphi^{c}+\beta\left(\pi^{c}-\varphi^{c}\right)=0,} \\
& \quad-\partial_{z} \square \varphi^{c}+\beta \partial_{z} \pi^{c}=0 \quad \text { (pseudoscalar sector) } .
\end{aligned}
$$

Note that the coupled differential equations in (49) for the pseudoscalar sector can be combined into the single differential equation

$$
\partial_{z}\left[\partial_{z}+A_{s}^{\prime}-b^{\prime}+(\ln \beta)^{\prime}\right] \Pi^{c}+(\square-\beta) \Pi^{c}=0,
$$

where we have defined $\Pi^{c} \equiv \partial_{z} \pi^{c}$.

\section{B. Vector mesons}

Let us start with the equation of motion describing the vector mesons, i.e., Eq. (47). Considering the Fourier transform on $V_{\mu}, V_{\mu}\left(x^{\mu}, z\right) \rightarrow V_{\mu}\left(k^{\mu}, z\right)$, then setting $\square \rightarrow m_{V}^{2}$, the resulting equation may be rewritten in the Schrödinger-like form through the transformation $V_{\mu}=\eta_{\mu} e^{-B_{V}} \psi_{v_{n}}$, where $2 B_{V}=A_{s}-b$ and $\eta_{\mu}$ is a (transverse) polarisation vector. The Schrödinger-like equation reads

$$
-\partial_{z}^{2} \psi_{v_{n}}+V_{V} \psi_{v_{n}}=m_{V}^{2} \psi_{v_{n}},
$$

where the potential is given by

$$
V_{V}=\left(\partial_{z} B_{V}\right)^{2}+\partial_{z}^{2} B_{V} .
$$

First, let us consider model IA where $b=a$. In this case, the solution of the eigenvalue problem is obtained numerically. A plot of the potential (52) for different values of $a_{0}$ is displayed on the left panel of Fig. 7. In turn, our numerical results for the masses as a function of $a_{0}$ are displayed on the left panel of Fig. 8 with solid lines. As can be seen, the masses are sensitive to the parameter $a_{0}$; special attention requires the mass of the ground state which decreases with the increasing of $a_{0}$, while the masses of the other states increase with the increasing of $a_{0}$. It is worth mentioning that in the limit of zero $a_{0}$, the results reduce to those obtained in the linear soft wall model displayed with dashed lines $[10,13,31,51]$. Note that the coupling $a(\Phi)$ reduces to $a(\Phi)=-a_{0} \Phi$ for models of type $\mathrm{I}$ in the limit of large $a_{0}$. This corresponds to an effective negative dilaton profile. As explained in Ref. [54], the potential of the Schrödingerlike equation is insensitive to the signal of the dilaton field, meaning that the spectrum will not change in relation to the original soft wall model. However, as shown in Ref. [54], the longitudinal fluctuation of the field $V_{\mu}$ will give rise to a 

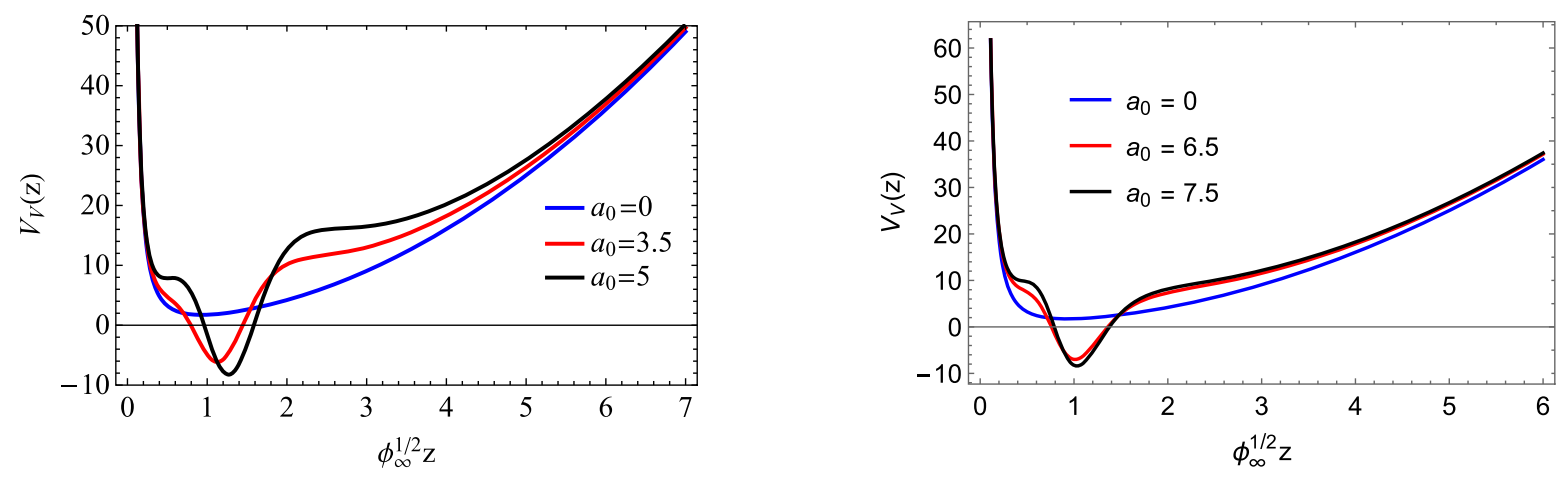

FIG. 7. Left: the potential of the Schrödinger-like equation for selected values of $a_{0}$ for models of type I. As can be seen, increasing $a_{0}$ the potential becomes negative. Right: the same as left panel for models of type II.
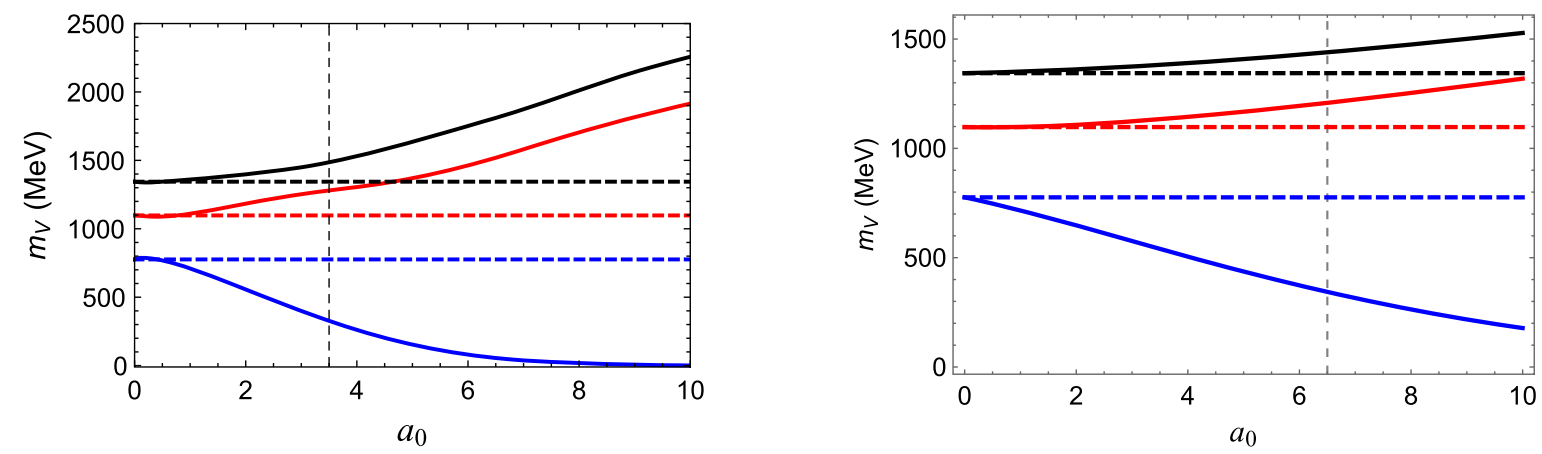

FIG. 8. Left: masses of vector mesons $M_{V}$ as functions of the parameter $a_{0}$ for models of type I; solid lines represent the results for $a=b$ (model IA), while dashed lines represent results for $a \neq b$ (model IB). Right: masses of vector mesons $M_{V}$ functions of the parameter $a_{0}$ for models of type II; solid lines represent the results for $a=b$ (model IIA), while dashed lines represent results for $a \neq b$ (model IIB).

massless state. In turn, in the models of type I, a massless mode arises naturally by solving the Schrödinger-like equation in the region of large $a_{0}$. The parameter $a_{0}$ controls the minimum of the potential well (see the left panel of Fig. 7), opening the possibility of the emergence of this state as can seen on the left panel of Fig. 8. Regardless of what the origin of the massless mode is, it is a nonphysical state and must be avoided. In the following analysis, we choose $a_{0}=3.5$, such that the ground state is not so light.

Now, consider models of type IB where $a \neq b$, with $b=$ $\Phi=\phi_{\infty} z^{2}$ and $a$ is given by (17). Note that $b$ is the dilaton field of the linear soft wall model. Hence, in this case, the problem has the exact solution [10]

$$
m_{V}^{2}=4 \phi_{\infty}(1+n), \quad n=0,1,2, \ldots
$$

The free parameter $\phi_{\infty}$ may be fixed by comparing the first state with the corresponding experimental result of the $\rho$ meson [66], providing us with the value $\phi_{\infty}=(388 \mathrm{MeV})^{2}$. In model IB, the potential corresponds to the case $a_{0}=0$ in the left panel of Fig. 7, while the masses as functions of $a_{0}$ are displayed in the left panel of Fig. 8 with dashed lines.
The results obtained above for models of type I can be extended for models of type II, which is described by Eq. (19). For model IIA, where $a=b$, the potential is displayed on the right panel of Fig. 7, while the masses as functions of $a_{0}$ are displayed on the right panel of Fig. 8 . The main difference between models of type IIA with respect to models of type IA is that the mass of the ground states decreases slowly with $a_{0}$. Note also that the results for model IIB ( $b=\Phi=\phi_{\infty} z^{2}$ ) are equivalent to results for model IB.

Finally, considering $a_{0}=3.5$ for models of type I and $a_{0}=6.5$ for models of type II, we calculated the mass of the vector mesons. We display our results and compare them against some results available in the literature in Table III. As can be seen from the table, excluding the ground state, our results for all models are close to the experimental results available from the Particle Data Group (PDG) [71]. Note also that the results for the ground state in models IB and IIB are the same and closer to the experimental result. This is because they are equivalent to the original soft wall model and the parameter $\phi_{\infty}$ was fixed in that model in order to match the experimental result for the $\rho$ meson mass. 
TABLE III. Masses of vector mesons (in MeV) obtained in models of type I for $a_{0}=3.5$, models of type II for $a_{0}=6.5$, compared against the holographic models [13] and experimental results from the PDG [71].

\begin{tabular}{ccccccc}
\hline \hline & $\begin{array}{c}\text { Model IA } \\
(a=b)\end{array}$ & $\begin{array}{c}\text { Model IB } \\
(a \neq b)\end{array}$ & $\begin{array}{c}\text { Model IIA } \\
(a=b)\end{array}$ & $\begin{array}{c}\text { Model IIB } \\
(a \neq b)\end{array}$ & GKK [13] & $\rho$ experimental [71] \\
\hline 0 & 327 & 776 & 344 & 776 & 475 & $776 \pm 1$ \\
1 & 1280 & 1097 & 1208 & 1097 & 1129 & $1282 \pm 37$ \\
2 & 1486 & 1344 & 1439 & 1344 & 1429 & $1465 \pm 25$ \\
3 & 1662 & 1552 & 1632 & 1552 & 1674 & $1720 \pm 20$ \\
4 & 1823 & 1735 & 1802 & 1735 & 1884 & $1909 \pm 30$ \\
5 & 2116 & 1901 & 1958 & 1901 & 2072 & $2149 \pm 17$ \\
6 & 2250 & 2053 & 2104 & 2053 & 2243 & $2265 \pm 40$ \\
\hline \hline
\end{tabular}

\section{Scalar mesons}

Let us move on to the scalar mesons, which are described by Eq. (46). Considering the Fourier transform on $S\left(x^{\mu}, z\right), S\left(x^{\mu}, z\right) \rightarrow S\left(k^{\mu}, z\right)$, then replacing $\square \rightarrow m_{s}^{2}$, and implementing the transformation $S=e^{-B_{S}} \psi_{S_{n}}(z)$, where $2 B_{S}=3 A_{s}-a$, the equation (46) may be rewritten in the Schrödinger-like form

$$
-\partial_{z}^{2} \psi_{s_{n}}+V_{S} \psi_{s_{n}}=m_{s}^{2} \psi_{s_{n}},
$$

where the potential is given by

$$
V_{S}=\left(\partial_{z} B_{S}\right)^{2}+\partial_{z}^{2} B_{S}+e^{2 A_{s}}\left(m_{X}^{2}+\frac{3 \lambda}{2} v^{2}(z)\right)
$$

Note that the scaling symmetry of the tachyon differential equation arises here due to the product $\lambda v^{2}$ in the potential. This means that the spectrum of scalar mesons depends only on $\tilde{v}=\sqrt{\lambda} v$ which in turn is independent of $\lambda$ as long as $\lambda>0$. The masses of scalar mesons depend on the quark mass due to the presence of the tachyon field in the potential. For $\lambda=0$, the potential (55) reduces to results available in the literature for the linear soft wall model; see, for instance, Ref. [16] (see also Ref. [12]). We find that, for fixed $a_{0}$, the masses of scalar mesons are monotonically increasing functions of the parameter $\tilde{m}_{q}=\sqrt{\lambda} m_{q}$. This means that for fixed $m_{q}(\lambda)$, they are increasing functions of $\lambda\left(m_{q}\right)$. This is consistent with the results found in Ref. [51] for the case $a_{0}=0$, where the dilaton coupling becomes minimal.

A plot of the masses as functions of $a_{0}$ is displayed on the left panel of Fig. 9 for $m_{q}=3.63 \times 10^{-4} \mathrm{MeV}$ (near the chiral limit), where solid lines are the results for models IA and IB, while dashed lines represent the result of the linear soft wall model given by Eq. (D7). Note that the mass of the ground state decreases with the increasing of $a_{0}$ up to $a_{0}^{c}$; then, it increases with the increasing of $a_{0}$. Thus, to avoid any zero mode in the scalar sector of models of type I, we set $a_{0}=3.5$, represented by the vertical dashed line. In turn, the numerical results of the masses as functions of the quark mass, for fixed $a_{0}$ and $\lambda$, are displayed on the left panel of Fig. 10, where solid lines represent the results for model IA, while dashed lines display the results for model IB. As expected, for fixed $a_{0}$ and $\lambda$, the masses of scalar mesons increase with the increasing of the quark mass $m_{q}$. Note that the states obtained in model IA are heavier than the states obtained in model IB.

The masses as functions of $a_{0}$ obtained in models of type II are displayed on the right panel of Fig. 9, for $m_{q}=$ $3.63 \times 10^{-4} \mathrm{MeV}$ (near the chiral limit). The results for model IIA are represented by solid lines, whereas the results for model IIB are represented by dashed lines. Again, the mass of the ground state decreases with the increasing of $a_{0}$ up to $a_{0}^{c}$; then, it increases with $a_{0}$. This behavior motivated us to the choice $a_{0}=6.5$ to avoid the zero mode, as we did in models of type I. Finally, for fixed $a_{0}$ and $\lambda$, the masses as functions of the quark mass are displayed on the right panel of Fig. 10; results for model IIA are represented with solid line, while results for model IIB are represented with dashed lines. As expected, the masses increase with the increasing of the quark mass. These results are in agreement with the results obtained in Ref. [51], where the nonlinear extension of the soft wall model is investigated. We would like to remark we do not find instabilities in the physical region, i.e., $m_{q}>0$, as was previously reported in the spectrum of the scalar mesons in extensions of the linear soft wall model [31] (see also Refs. [21,51]).

For the fixed set of parameters, described in Table II, we may calculate the spectrum provided by the holographic models and compare the results against some results available in the literature. In Table IV, we show our results for model IA setting $m_{q}=9 \mathrm{MeV}$, model IB setting $m_{q}=4.7 \mathrm{MeV}$, model IIA setting $m_{q}=9.8 \mathrm{MeV}$, and model IIB setting $m_{q}=26.8 \mathrm{MeV}$. Each particular choice of the quark masses will be justified below, and we will see that these values are in agreement with the pion mass in the pseudoscalar mesons.

\section{Axial-vector mesons}

The axial-vector mesons are described by Eq. (48). Considering the Fourier transform on $A_{\perp}^{\mu}\left(x^{\mu}, z\right)$, 

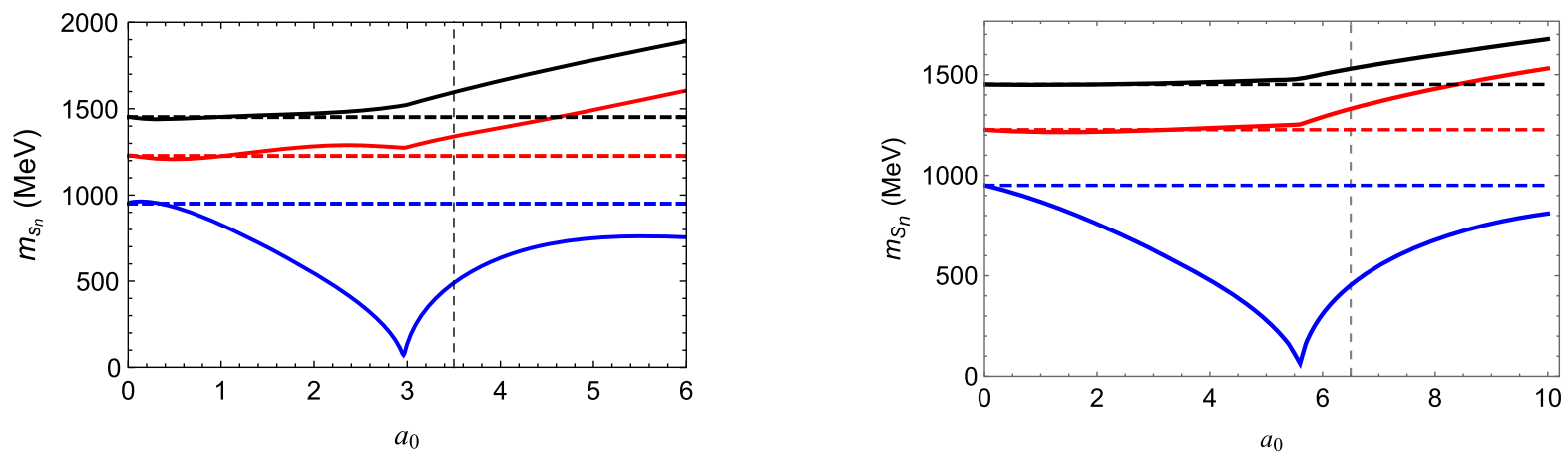

FIG. 9. Left: masses of scalar mesons as functions of the parameter $a_{0}$. Solid lines represent the results for models IA and IB, while dashed lines represent the results of the linear soft model. Right: masses of scalar mesons as functions of $a_{0}$. Solid lines represent the results for models IIA and IIB, while dashed lines represent the results of the linear soft model. These results were obtained setting $m_{q}=3.63 \times 10^{-4} \mathrm{MeV}$ (near the chiral limit).
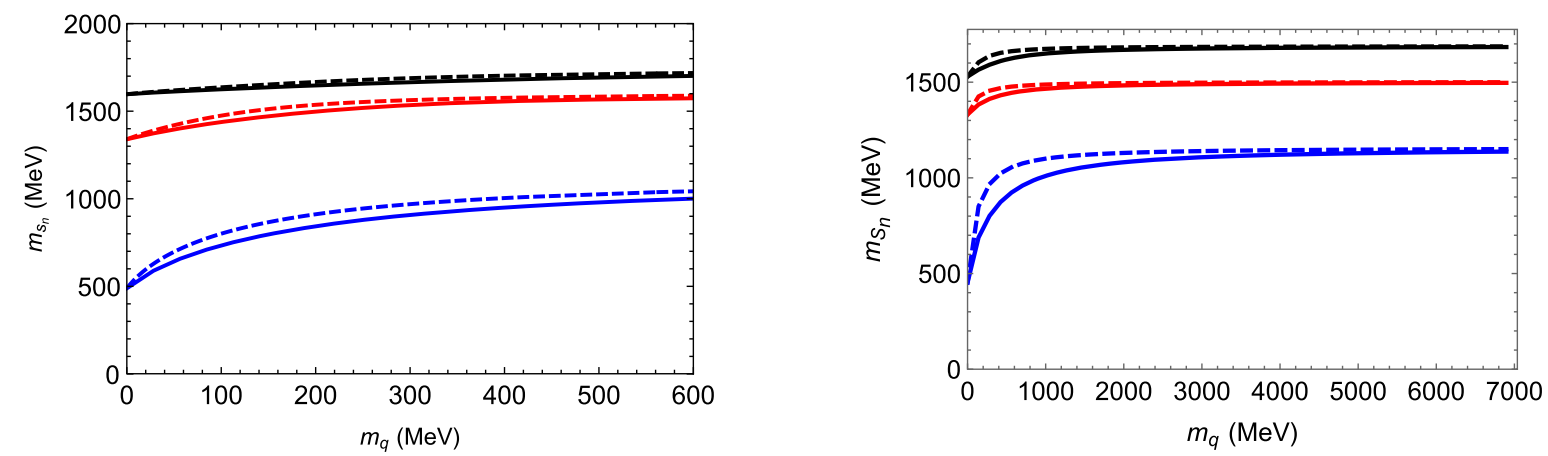

FIG. 10. Left: masses of scalar mesons as functions of the quark mass. Solid lines represent the results for model IA $(a=b)$ and $\lambda=160$, while dashed lines represent results for model IB $(a \neq b)$ and $\lambda=380$, and we set $a_{0}=3.5$. Right: masses of scalar mesons as functions of the quark mass. Solid lines represent the results for model IIA $(a=b)$ and $\lambda=60$, while dashed lines represent results for model IIB $(a \neq b)$ and $\lambda=413$, and we set $a_{0}=6.5$.

TABLE IV. Masses of scalar mesons (in MeV) obtained in the models of type I and models of type II, compared against the holographic model of Ref. [51], and experimental data from the PDG [71]. To get these results, we have considered $\lambda=160$ for model IA and $\lambda=380$ for model IB, while the quark masses are $m_{q}=9 \mathrm{MeV}$ and $m_{q}=4.7 \mathrm{MeV}$, respectively. For model IIA, we have considered $\lambda=60$, while the quark mass is $m_{q}=9.8 \mathrm{MeV}$, and for model IIB, $\lambda=413$ and $m_{q}=26.8 \mathrm{MeV}$.

\begin{tabular}{lcccccc}
\hline \hline & $\begin{array}{c}\text { Model IA } \\
(a=b)\end{array}$ & $\begin{array}{c}\text { Model IB } \\
(a \neq b)\end{array}$ & $\begin{array}{c}\text { Model IIA } \\
(a=b)\end{array}$ & $\begin{array}{c}\text { Model IIB } \\
(a \neq b)\end{array}$ & BM [51] & $f_{0}$ experimental [71] \\
\hline 0 & 526 & 519 & 539 & 546 & 980 & $990 \pm 20$ \\
1 & 1351 & 1349 & 1348 & 1350 & 1246 & $1350 \pm 150$ \\
2 & 1600 & 1599 & 1540 & 1541 & 1466 & $1505 \pm 6$ \\
3 & 1755 & 1755 & 1718 & 1719 & 1657 & $1724 \pm 7$ \\
4 & 1904 & 1904 & 1881 & 1881 & 1829 & $1992 \pm 16$ \\
5 & 2048 & 2048 & 2032 & 2032 & 1986 & $2103 \pm 8$ \\
6 & 2185 & 2185 & 2174 & 2174 & 2132 & $2314 \pm 25$ \\
7 & 2315 & 2315 & 2313 & 2313 & 2268 & \\
\hline \hline
\end{tabular}

$A_{\perp}^{\mu}\left(x^{\mu}, z\right) \rightarrow A_{\perp}^{\mu}\left(k^{\mu}, z\right)$, then replacing $\square \rightarrow m_{A_{n}}^{2}$, one may write the Schrödinger-like equation by redefining the axialvector field as $A_{\mu}=\eta_{\mu} e^{-B_{A}} \psi_{a_{n}}$, where $2 B_{A}=A_{s}-b$ and $\eta_{\mu}$ is a polarization vector; thus, we get

$$
-\partial_{z}^{2} \psi_{a_{n}}+V_{A} \psi_{a_{n}}=m_{A_{n}}^{2} \psi_{a_{n}},
$$

where the potential is given by 

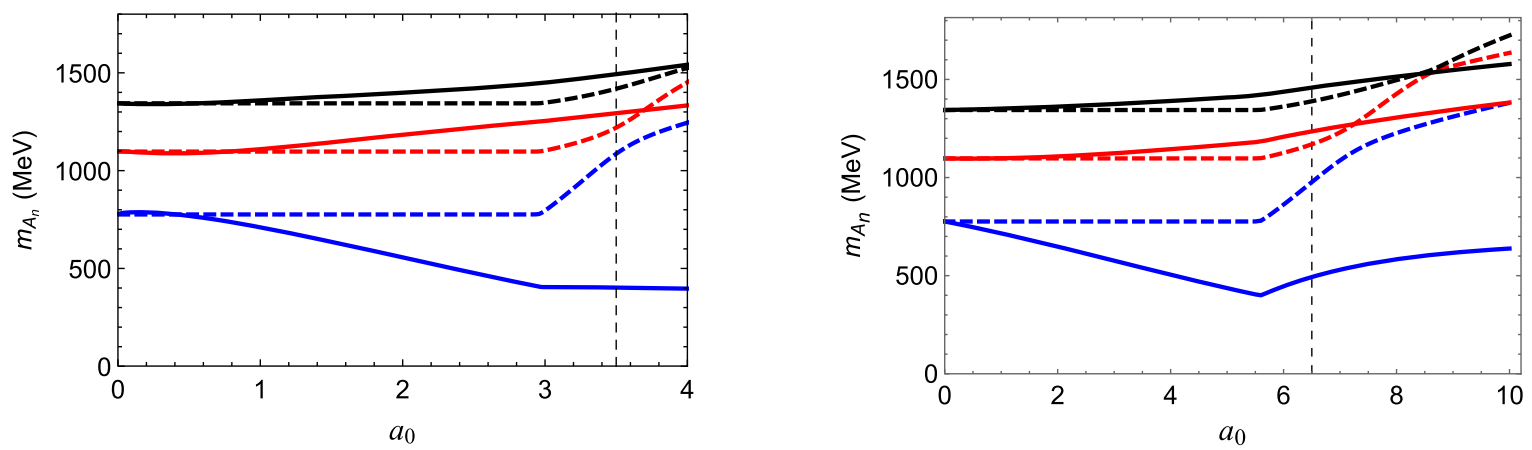

FIG. 11. Left: masses of axial-vector mesons as functions of $a_{0}$. Solid lines represent the results for model IA $(a=b)$ and $\lambda=160$, while dashed lines represent results for model IB $(a \neq b)$ and $\lambda=380$. Right: masses of axial-vector mesons as functions of $a_{0}$. Solid lines represent the results for model IIA $(a=b)$ and $\lambda=60$, while dashed lines represent results for model IIB $(a \neq b)$ and $\lambda=413$. These results were obtained setting $m_{q}=3.63 \times 10^{-4} \mathrm{MeV}$ (near the chiral limit).

$$
V_{A}=\left(\partial_{z} B_{A}\right)^{2}+\partial_{z}^{2} B_{A}+\beta
$$

and $\beta=g_{5}^{2} v^{2} e^{2 A_{s}+b-a}$. Note that the potential depends explicitly on the functions $a$ and $b$ and depends on $\lambda$ and $m_{q}$ through the tachyonic field $v=\tilde{v} / \sqrt{\lambda}$. Analogous to what we have done before, we solve the eigenvalue problem numerically. Our numerical results for the masses as functions of $a_{0}$ at fixed $m_{q}=3.63 \times 10^{-4} \mathrm{MeV}$ (near the chiral limit) are displayed on the left panel of Fig. 11 for model IA $(a=b)$ with solid lines and model IB $(a \neq b)$ with dashed lines. Similarly to the vectorial case, the mass of the ground state decreases with $a_{0}$. In the limit $\left(a_{0}, m_{q}\right) \rightarrow 0$, the function $\beta$ and the field $v$ go to zero. Hence, the Schrödinger potentials for the vectorial and axial sector coincide in that limit, i.e., $V_{A}=V_{V}$, meaning that the vector and axial-vector states are degenerate (chiral symmetry is restored). The masses as functions of $a_{0}$ for models of type II are displayed on the right panel of Fig. 11, where solid lines are results for model IIA, while dashed lines are results for model IIB. Those results were also obtained setting

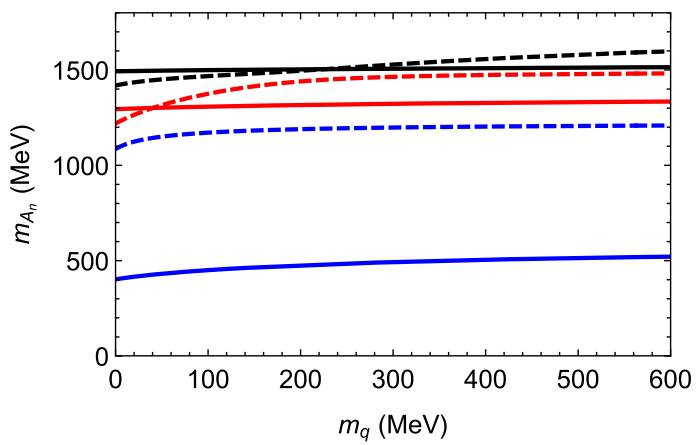

$m_{q}=3.63 \times 10^{-4} \mathrm{MeV}$ (near the chiral limit) and are similar to the ones found for models of type I.

Having fixed the parameter $a_{0}=3.5$ for models of type I, one may calculate the mass solving the eigenvalue problem numerically. The masses of axial-vector mesons as functions of the quark mass, for fixed $a_{0}$ and $\lambda$, are displayed in the left panel of Fig. 12, results for model IA are represented with solid lines, while results for model IB are represented with dashed lines. As expected, the masses increases with the increasing of the quark mass. However, note that the resonances become less sensitive to the quark mass in the heavy quark regime. These conclusions are also true for the results obtained in models of type II, displayed on the right panel of Fig. 12 for $a_{0}=6.5$ and selected values of $\lambda$. These results are in qualitative agreement with the results obtained in Ref. [51].

Finally, for the set of parameters displayed in Table II, we may calculate the spectrum provided by our models and compare them against the results available in the literature. In Table V, we write our results for model IA setting $m_{q}=9 \mathrm{MeV}$, model IB setting $m_{q}=4.7 \mathrm{MeV}$, model IIA setting $m_{q}=9.8 \mathrm{MeV}$, and model IIB setting

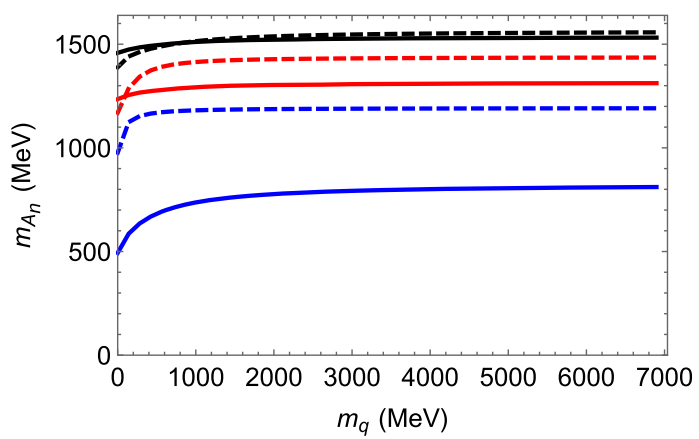

FIG. 12. The mass of the axial-vector mesons as a function of the quark mass. Solid lines represent the results for model IA $(a=b)$ and $\lambda=160$, while dashed lines represent results for model IB $(a \neq b)$ and $\lambda=380$, and we set $a_{0}=3.5$. Right: the mass of the axialvector mesons as a function of the quark mass. Solid lines represent the results for model IIA $(a=b)$ and $\lambda=60$, while dashed lines represent results for model IIA $(a \neq b)$ and $\lambda=413$, and we set $a_{0}=6.5$. 
TABLE V. The masses of the axial-vector mesons (in MeV) obtained in the models of type I and models of type II, compared against the holographic model of Ref. [13] and experimental data [71]. We have considered $\lambda=160$ for model IA and $\lambda=380$ for model IB, while the quark masses are $m_{q}=9 \mathrm{MeV}$ and $m_{q}=4.7 \mathrm{MeV}$, respectively. For model IIA, we have considered $\lambda=60$, while the quark mass is $m_{q}=9.8 \mathrm{MeV}$, and for model IIB, $\lambda=413$ and $m_{q}=26.8 \mathrm{MeV}$.

\begin{tabular}{ccccccc}
\hline \hline & $\begin{array}{c}\text { Model IA } \\
(a=b)\end{array}$ & $\begin{array}{c}\text { Model IB } \\
(a \neq b)\end{array}$ & $\begin{array}{c}\text { Model IIA } \\
(a=b)\end{array}$ & $\begin{array}{c}\text { Model IIB } \\
(a \neq b)\end{array}$ & GKK [13] & $a_{1}$ experimental [71] \\
\hline 0 & 409 & 1098 & 525 & 1105 & 1185 & $1230 \pm 40$ \\
1 & 1296 & 1231 & 1242 & 1261 & 1591 & $1647 \pm 22$ \\
2 & 1494 & 1423 & 1463 & 1431 & 1900 & $1930_{-70}^{+30}$ \\
3 & 1669 & 1625 & 1651 & 1625 & 2101 & $2096 \pm 122$ \\
4 & 1828 & 1797 & 1817 & 1798 & 2279 & $2270_{-40}^{+55}$ \\
5 & 1978 & 1950 & 1970 & 1954 & & \\
6 & 2316 & 2092 & 2114 & 2100 & & \\
\hline \hline
\end{tabular}

$m_{q}=26.8 \mathrm{MeV}$. Each particular choice of the quark masses will be justified bellow, and we will see that these values lead to a pion mass in agreement with experimental data. We observe that the results provided by models IB and IIB are closer to experimental data compared with the results provided by models IA and IIA.

\section{E. Pseudoscalar mesons}

We now focus in the pseudoscalar sector. As we did in previous subsections, we may rewrite the coupled differential equations (50) in the Schrödinger-like form. However, we realized that the ground state is very sensitive numerically; for that reason, we change our strategy and solve the coupled differential equations (50) directly. Introducing the Fourier transform $\pi(x, z) \rightarrow \pi(k, z)$ and $\varphi(x, z) \rightarrow \varphi(k, z)$, then replacing $\square \rightarrow m_{\pi_{n}}^{2}$ in Eq. (50), we get

$$
\begin{array}{r}
{\left[\partial_{z}+A_{s}^{\prime}-b^{\prime}\right] \partial_{z} \varphi+\beta(\pi-\varphi)=0,} \\
-m_{\pi_{n}}^{2} \partial_{z} \varphi+\beta \partial_{z} \pi=0,
\end{array}
$$

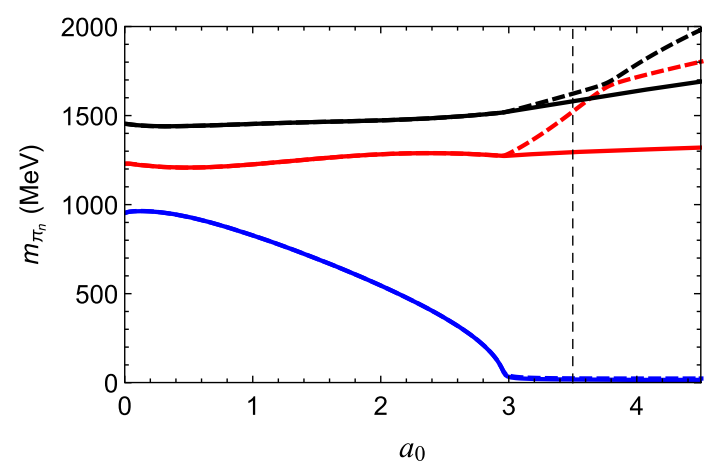

where $\beta=g_{5}^{2} v^{2} e^{2 A_{s}+b-a}$. The eigenvalue problem is solved numerically using the shooting method. Our results for the masses as functions of $a_{0}$ are displayed in the left panel of Fig. 13, where results for model IA are represented with solid lines, while results for model IB are represented with dashed lines, and we set $m_{q}=3.63 \times 10^{-4} \mathrm{MeV}$ (near the chiral limit). Note that the mass of the ground state decreases with the increasing of $a_{0}$ up to $a_{0}^{c}$; then, it lies very close to zero, representing the Nambu-Goldstone boson arising for $a_{0} \geq a_{0}^{c}$ with $a_{0}^{c} \approx 2.97$ the same value found in Sec. III E from the analysis of the chiral condensate. In turn, the masses of pseudoscalar resonances display a different behavior with increasing of $a_{0}$. The same conclusions are true for models of type II, displayed on the right panel of Fig. 13, where solid lines represent results for model IIA, while dashed lines represent results for model IIB. The Nambu-Goldstone state appears for $a_{0} \geq a_{0}^{c}$ with $a_{0}^{c} \approx 5$.6, the same value found in Sec. III E from the analysis of the chiral condensate.

Therefore, the choice of the parameter $a_{0}=3.5$ for models of type I and $a_{0}=6.5$ for models of type II is well

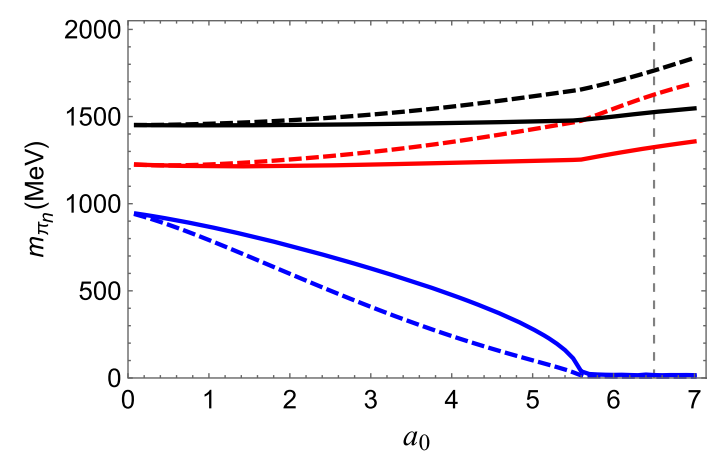

FIG. 13. Left: the mass of the pseudoscalar mesons as a function of $a_{0}$. Solid lines represent the results for model IA $(a=b)$ and $\lambda=160$, while dashed lines represent results for model IB $(a \neq b)$ and $\lambda=380$. Right: the mass of the pseudoscalar mesons as a function of $a_{0}$. Solid lines represent the results for model IIA $(a=b)$ and $\lambda=60$, while dashed lines represent results for model IIB $(a \neq b)$ and $\lambda=413$. These results were obtained setting $m_{q}=3.63 \times 10^{-4} \mathrm{MeV}$. 

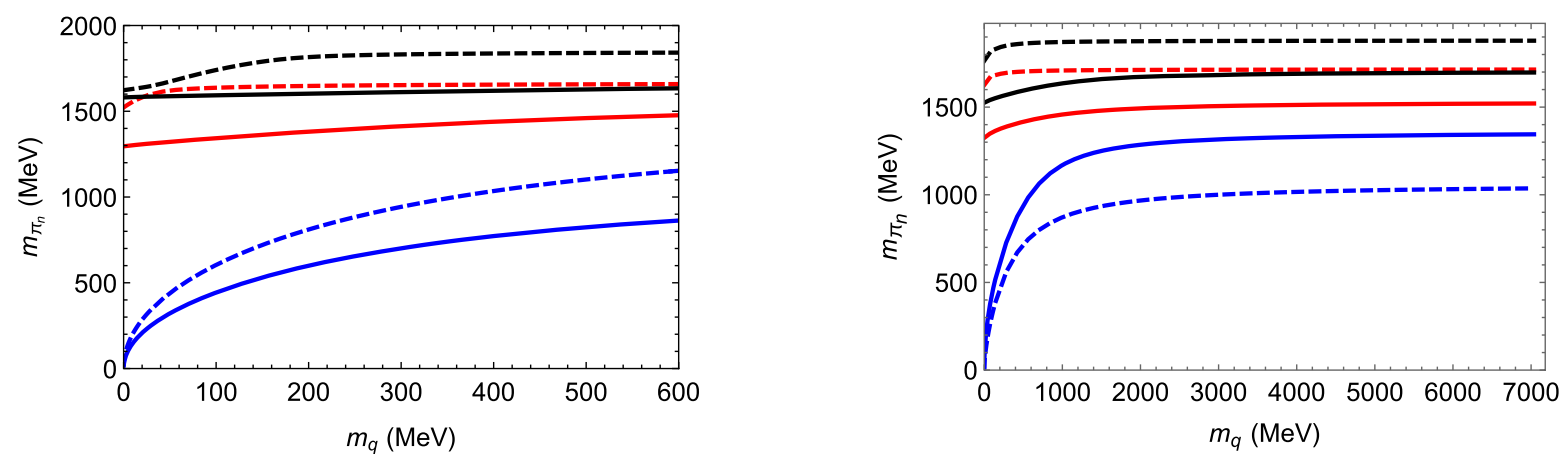

FIG. 14. Left: masses of pseudoscalar mesons as functions of the quark mass. Solid lines represent the results for model IA $(a=b)$ and $\lambda=160$, while dashed lines represent results for model IB $(a \neq b)$ and $\lambda=380$, and we set $a_{0}=3.5$. Right: masses of pseudoscalar mesons as functions of the quark mass. Solid lines represent the results for model IIA $(a=b)$ and $\lambda=60$, while dashed lines represent the results for model IIB $(a \neq b)$ and $\lambda=413$, and we set $a_{0}=6.5$.

justified by the scalar sector and pseusoscalar sectors. This choice allowed us to avoid a zero mode in the scalar sector and find a Nambu-Goldstone boson in the pseudoscalar sector in the chiral limit. Masses of pseudoscalar mesons as functions of the quark mass, for fixed $a_{0}$ and $\lambda$, are displayed on the left panel of Fig. 14, where results for model IA are represented with solid lines, while results for model IB are represented with dashed lines. As expected, the mass of the ground state approaches zero near the chiral limit, while the masses of the resonances approach finite values. All the masses increase with the increasing of the quark mass. However, note that the masses of pseudoscalar resonances are less sensitive to the quark mass in the heavy quark regime. These conclusions are also true for results obtained in models of type II, displayed on the right panel of Fig. 14.

Finally, for the set of parameters displayed in Table II, we calculate the spectrum provided by our models and compare them against the results available in the literature. In Table VI we show our results for model IA setting $m_{q}=9 \mathrm{MeV}$, model IB setting $m_{q}=4.7 \mathrm{MeV}$, model IIA setting $m_{q}=9.8 \mathrm{MeV}$, and model IIB setting $m_{q}=26.8 \mathrm{MeV}$. Each particular choice of the quark masses is justified by the fact that it allowed us to reproduce the experimental result for the pion mass.

So far, we have found a consistent description of spontaneous chiral symmetry breaking because the chiral condensate is nonzero in the chiral limit, and a NambuGoldstone state arises in the spectrum of pseudoscalar mesons, which is consistent with the pion. There are other important tests of consistency that we want to show, the behavior of meson decay constants and the GOR relation. This is done in the next section.

\section{DECAY CONSTANTS}

To complement the analysis presented above, we now calculate the decay constants of vector, axial-vector, scalar, and pseudoscalar mesons. Details on the derivation of the holographic dictionary are presented in Appendix C (see also Refs. [24,51]). We will be particularly interested in the pseudoscalar sector where we expect to confirm the presence of Nambu-Goldstone bosons in the chiral limit. We will fulfill this expectation and will also reproduce the GOR relation near the chiral limit.

TABLE VI. Masses of pseudoscalar mesons (in MeV) obtained in models of type I and models of type II, compared against the soft wall model [10], the holographic model of Ref. [72], and experimental data [71]. We have considered $\lambda=160$ for models of type I and $\lambda=380$ for models of type II, while the quark masses are $m_{q}=9 \mathrm{MeV}$ and $m_{q}=4.79 \mathrm{MeV}$, respectively. For model IIA, we have considered $\lambda=60$, while the quark mass is $m_{q}=9.8 \mathrm{MeV}$, and for model IIB, $\lambda=413$ and $m_{q}=26.8 \mathrm{MeV}$.

\begin{tabular}{ccccccc}
\hline \hline$n$ & $\begin{array}{c}\text { Model IA } \\
(a=b)\end{array}$ & $\begin{array}{c}\text { Model IB } \\
(a \neq b)\end{array}$ & $\begin{array}{c}\text { Model IIA } \\
(a=b)\end{array}$ & $\begin{array}{c}\text { Model IIB } \\
(a \neq b)\end{array}$ & KBK [72] & $\pi$ experimental [71] \\
\hline 0 & 140 & 140 & 140 & 140 & 144 & 140 \\
1 & 1301 & 1539 & 1338 & 1675 & 1557 & $1300 \pm 100$ \\
2 & 1582 & 1626 & 1533 & 1819 & 1887 & $1816 \pm 14$ \\
3 & 1739 & 1794 & 1713 & 1945 & 2090 & 2070 \\
4 & 1890 & 1945 & 1877 & 2183 & 2270 & 2360 \\
5 & 2036 & 2083 & 2028 & 2301 & 2434 & 2586 \\
6 & 2175 & 2212 & 2170 & 2422 & 250 & \\
\hline \hline
\end{tabular}




\section{A. Vector mesons}

In holographic models for QCD, the decay constants are related to the normalization constants arising from the normalization solutions of the eigenvalue problems. The normalization condition for the vector mesons is given by

$\int d z \psi_{v_{m}}(z) \psi_{v_{n}}(z)=\int d z e^{A_{s}-b} v_{m}(z) v_{n}(z)=\delta_{m n}$,

where $A_{s}=-\ln z$ is the AdS warp factor, $b$ is the dilaton coupling to the gauge fields, $\psi_{v_{n}}$ is the wave function of the Schrödinger equation (51), and $v_{n}$ is the normalizable solution in the vectorial sector related to $\psi_{v_{n}}$ by $v_{n}(z)=e^{-B_{V}} \psi_{v_{n}}(z)$. As described in Appendix $\mathrm{C}$, the meson decay constants are given by Eq. (C7). For the vectorial sector, the decay constants reduce to the following formula,

$$
F_{v_{n}}=\left.\lim _{\epsilon \rightarrow 0} \frac{e^{A_{s}-b}}{g_{5}} \partial_{z} v_{n}\right|_{z=\epsilon}=\frac{2}{g_{5}} N_{v_{n}}
$$

where we have defined the normalization constant $N_{v_{n}}$, as the coefficient which appears in the UV expansion of the vector mode, i.e., $v_{n}(z)=N_{v_{n}} z^{2}+\cdots$. Hence, the decay constants are proportional to the normalization constants. Therefore, solving numerically the Schrödinger-like equation and using the normalization condition, we are able to calculate the decay constants of the vector mesons. We display our numerical results for the vector meson decay constants as functions of $a_{0}$ on the left panel of Fig. 15, where solid lines represent results for model IA, while dashed lines represent results for model IB. As illustrated by the figure, the results for model IA have a peculiar behavior changing the hierarchy with the increasing of $a_{0}$. In contrast, the results provided by model IB do not change with $a_{0}$. In fact, the results for model IB are the same as obtained in the linear soft wall model because in that case the dilaton coupling $b$ is minimal. In that particular case, we find an

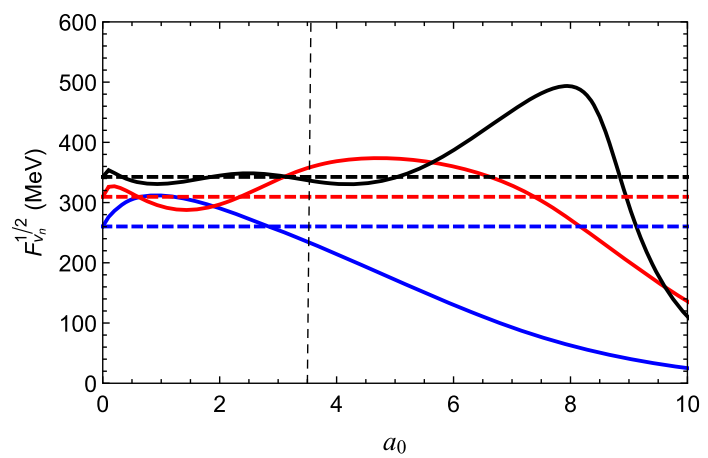

analytic solution for the decay constants in the linear soft wall model, derived in Appendix D.

Our numerical results for models of type II are displayed on the right panel of Fig. 15, where solid lines represent results for model IIA, while dashed line represent results for model IIB. From the figure, we note that the decay constants obtained in model IIA are smoother than those obtained in model IA, meaning that the decay constants are sensitive to the form of the interpolation functions (17) and (19). Finally, for the set of parameters displayed in Table II, although in the vectorial case the results are independent of $\lambda$, we calculate the corresponding values of the vector meson decay constants. The results are displayed in Table VII, compared with experimental results of Ref. [73]. Note that there is a change in the hierarchy of vector meson decay constants $F_{V^{n}}$ in model IA. The other models display the ordinary hierarchy $F_{V^{0}}<F_{V^{1}}<F_{V^{2}}$ found in previous works.

\section{B. Scalar mesons}

As we did in our previous subsection, we start with the normalization condition. For the scalar sector, it is given by the kinetic term related to scalar fluctuation of the Lagrangian (B6), which is

$\int d z \psi_{s_{m}}(z) \psi_{s_{n}}(z)=\int d z e^{3 A_{s}-a} S_{m}(z) S_{n}(z)=\delta_{m n}$.

Considering the UV expansion of the normalizable solution of $S_{n}(z)$, which is given by $S_{n}(z)=N_{s_{n}} z^{3}+\cdots$, here $N_{S_{n}}$ is the normalization constant calculated by plugging the solution in (61). Thus, the decay constants are given by $(\mathrm{C} 7)$

$$
F_{S_{n}}=\left.\zeta z e^{3 A_{s}-a} \partial_{z} S_{n}\right|_{z=\epsilon}=3 \zeta N_{s_{n}} .
$$

As expected, the meson decay constants are proportional to the corresponding normalization constants. We display the results for the scalar meson decay constants as functions of $a_{0}$ on the left panel of Fig. 16, for $m_{q}=3.63 \times 10^{-4} \mathrm{MeV}$

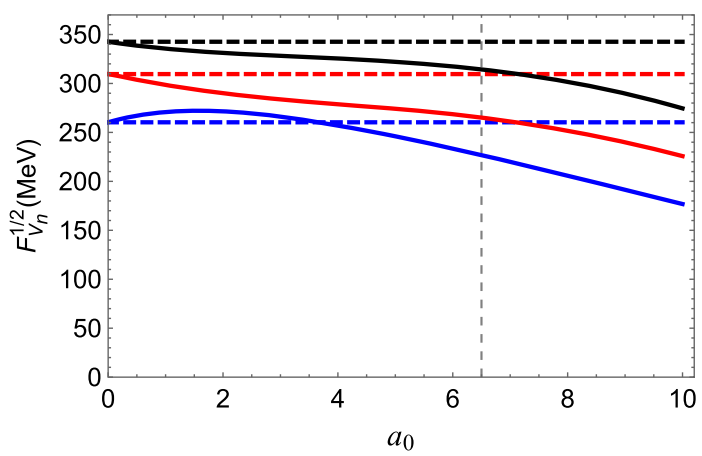

FIG. 15. Left: decay constants of the vector mesons as a function of $a_{0}$ obtained in models of type I, where solid lines represent results obtained in model IA $(a=b)$, while dashed lines represent results obtained in model IB $(a \neq b)$. Right: the same as left panel for models of type II. 
TABLE VII. The decay constants (in MeV) obtained in the models of type I and II, compared against the result obtained in the linear soft wall model [10] and experimental results of [73]. These results were found for the set of parameters displayed in Table II.

\begin{tabular}{lcccccc}
\hline \hline $\begin{array}{c}\text { Model IA } \\
(a=b)\end{array}$ & $\begin{array}{c}\text { Model IB } \\
(a \neq b)\end{array}$ & $\begin{array}{c}\text { Model IIA } \\
(a=b)\end{array}$ & $\begin{array}{c}\text { Model IIB } \\
(a \neq b)\end{array}$ & SW [10] & $\begin{array}{c}\text { Experimental [73] } \\
\left(F_{V^{a}}=g_{\rho}\right)\end{array}$ \\
\hline$F_{V_{0}}^{1 / 2}$ & 235 & 260 & 226 & 260 & 261 & $346.2 \pm 1.4$ \\
$F_{V_{1}}^{1 / 2}$ & 357 & 310 & 265 & 310 & & $433 \pm 13$ \\
$F_{V_{2}}^{1 / 2}$ & 337 & 343 & 314 & 343 & & \\
\hline \hline
\end{tabular}

(near the chiral limit). In fact, in the scalar sector, the results for model IA and model IB are the same, represented with solid lines in the figure. The figure also shows that the hierarchy in certain intervals of the parameter $a_{0}$ changes. We highlight the vertical dashed line at $a_{0}=3.5$, the fixed value used in this paper in models of type I. We also plot the analytic results obtained in the linear soft wall model (see Appendix D) with dashed lines. The corresponding results for models of type II are displayed on the right panel of Fig. 16. Interestingly, the hierarchy does not change with $a_{0}$, and the results are smoother than those obtained in models of type I; we also highlight the vertical dashed line for $a_{0}=6.5$, the fixed value used for models of type II.

The scalar meson decay constants as functions of the quark mass are displayed on the left panel of Fig. 17, where solid lines represent results for model IA, while dashed lines represent results for model IB. Note the change in the hierarchy of decay constants near the chiral limit; this is consistent with the left panel of Fig. 16 (see the vertical dashed line). In both models of type I, the scalar meson decay constants decrease with the increasing of the quark mass, and the hierarchy is restored in the regime of heavy quarks. The corresponding results for models of type II are displayed on the right panel of Fig. 17, where solid lines represent results for model IIA, while dashed lines represent results for model IIB. As the figures illustrates, the scalar meson decay constants for models of type II decrease

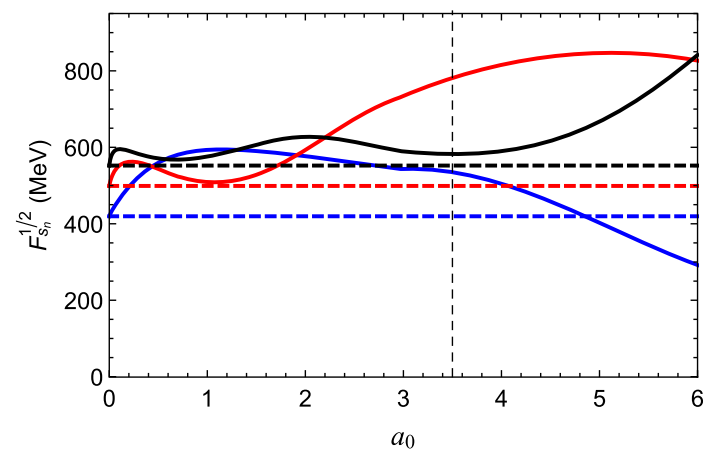

with the increasing of the quark mass, and there is a change in the hierarchy in the regime of heavy quarks.

Finally, we show our results for the decay constants at the specific values of the parameters given in Table II. The results are displayed in Table VIII. The quark masses were fixed as $m_{q}=9 \mathrm{MeV}, m_{q}=4.7 \mathrm{MeV}, m_{q}=9.8 \mathrm{MeV}$, and $m_{q}=26.8 \mathrm{MeV}$ for models IA, IB, IIA, and IIB, respectively.

\section{Axial-vector mesons}

We follow the same procedure described above this time for the axial-vector mesons, where the normalization condition is given by

$\int d z \psi_{a_{m}}(z) \psi_{a_{n}}(z)=\int d z e^{A_{s}-b} a_{m}(z) a_{n}(z)=\delta_{m n}$,

where $a_{n}(z)=e^{-B_{A}} \psi_{a_{n}}(z)$ is the axial-vector normalizable solution and $\psi_{a_{n}}(z)$ is the corresponding wave function of the Schrödinger-like equation (56). Plugging the UV expansion of $a_{n}(z)$, which is given by $a_{n}(z)=N_{a_{n}} z^{2}+\cdots$, where $N_{a_{n}}$ is the normalization constant, in (C7), we obtain the axial-vector meson decay constants

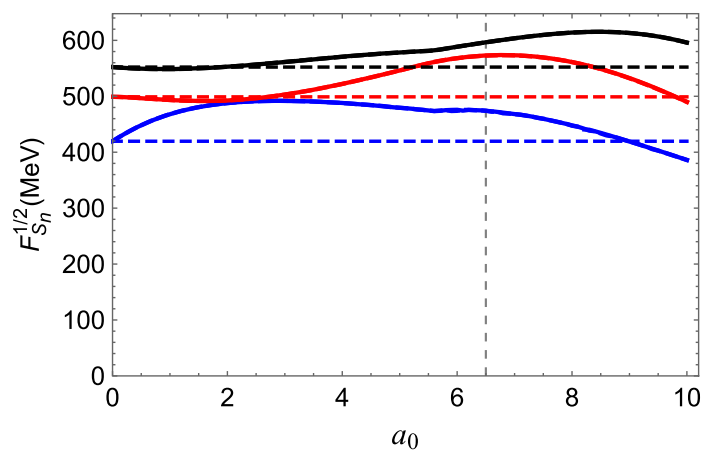

FIG. 16. Left: decay constants of scalar mesons as functions of $a_{0}$ for models of type I. Right: decay constants of scalar mesons as functions of $a_{0}$ for models of type II. We get these results for $m_{q}=3.63 \times 10^{-4} \mathrm{MeV}$ (near the chiral limit) in both type of models. Horizontal dashed lines represent the analytic results obtained in the linear soft wall model; see Appendix D. Vertical dashed lines represent the fixed values $a_{0}=3.5$ and $a_{0}=6.5$ for models of type I and II, respectively. 

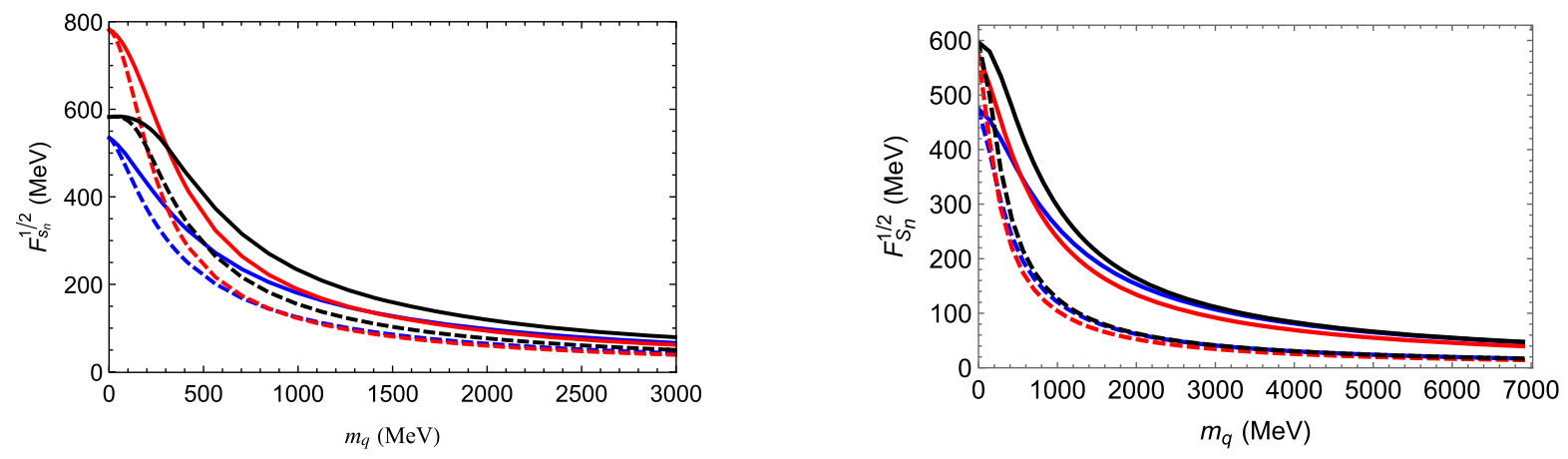

FIG. 17. Left: decay constants of scalar mesons as functions of $m_{q}$ in models of type I with $a_{0}=3.5$; solid lines represent the results for model IA $(\lambda=160)$, while dashed lines represent results for model IB $(\lambda=380)$. Right: decay constants of scalar mesons as functions of $m_{q}$ for models of type II with $a_{0}=6.5$; solid lines represent the results for model IIA $(\lambda=60)$, while dashed lines represent results for model IIB $(\lambda=413)$.

$$
F_{a_{n}}=\left.\lim _{\epsilon \rightarrow 0} \frac{e^{A_{s}-b}}{g_{5}} \partial_{z} a_{n}\right|_{z=\epsilon}=\frac{2}{g_{5}} N_{a_{n}} .
$$

Thus, the problem of finding decay constants has been reduced to the calculation of normalization constants. Our numerical results for the axial-vector meson decay constants for $m_{q}=3.63 \times 10^{-4} \mathrm{MeV}$ (near the chiral limit) as functions of $a_{0}$ are displayed on the left panel of Fig. 18, where solid lines represent results for model IA, while dashed lines represent results for model IB. As illustrated by the figure, the decay constants in model IA have a peculiar behavior changing the hierarchy when $a_{0}$ increases. The decay constants in model IB do not change with $a_{0}$ up to $a_{0}=a_{0}^{c}$; then, they vary with $a_{0}$ faster than the decay constants in model IA. The results provided by models of type II are displayed on the right panel of Fig. 18, where results for model IIA (IIB) are represented by solid lines (dashed lines). Note that the variation of decay constants with $a_{0}$ in model IIA is smoother than the variation in model IA. On the other hand, the decay constants in models IB and IIB change rapidly for $a_{0} \geq a_{0}^{c}$.

Having fixed the parameter $a_{0}=3.5$ for models of type I, we calculated the decay constants of axial-vector mesons as for different values of the quark mass. Our numerical results are displayed in the left panel of Fig. 19, where solid lines (dashed lines) represent results for model IA (IB). As shown in the figure, the behavior of the decay constants is different for each state in the region of small quark mass. There is a change in hierarchy between the second and third states near the chiral limit, which is consistent with the results displayed on the left panel of Fig. 18 (see the vertical dashed line). The hierarchy is restored in the regime of heavy quarks, where we have $F_{A_{0}}^{1 / 2}<F_{A_{1}}^{1 / 2}<F_{A_{2}}^{1 / 2}$; this result is in qualitative agreement with the results reported in Ref. [51]. The results obtained in models of type II are displayed on the right panel of Fig. 19, where solid lines (dashed lines) represent results for model IIA (IIB). In model IIA, the hierarchy between decay constants is preserved near the chiral limit but slightly changes in the heavy quark regime. In model IIB, the hierarchy is not preserved near the chiral limit, but it is restored in the regime of heavy quarks, where we have $F_{A_{0}}^{1 / 2}<F_{A_{1}}^{1 / 2}<F_{A_{2}}^{1 / 2}$.

For the fixed set of parameters displayed in Table II, we calculate the corresponding values of axial-vector meson decay constants and display the results in Table IX. The quark masses were fixed as $m_{q}=9 \mathrm{MeV}, m_{q}=4.7 \mathrm{MeV}$, $m_{q}=9.8 \mathrm{MeV}$, and $m_{q}=26.8 \mathrm{MeV}$ for models of type IA, IB, IIA, and IIB, respectively.

\section{Pseudoscalar mesons}

For the pseudoscalar mesons, the normalization condition in terms of the normalizable solution $\varphi_{n}(z)$ is given by

TABLE VIII. Decay constants of scalar mesons (in MeV) obtained in models of type I and II for the set of parameters given in Table II, compared against the result obtained in the linear soft wall model [10] and the result obtained in QCD [74]. The quark masses were fixed as $m_{q}=9 \mathrm{MeV}, m_{q}=4.7 \mathrm{MeV}, m_{q}=9.8 \mathrm{MeV}$, and $m_{q}=$ $26.8 \mathrm{MeV}$ for models IA, IB, IIA, and IIB, respectively.

\begin{tabular}{lcccccc}
\hline \hline & $\begin{array}{c}\text { Model IA } \\
(a=b)\end{array}$ & $\begin{array}{c}\text { Model IB } \\
(a \neq b)\end{array}$ & $\begin{array}{c}\text { Model IIA } \\
(a=b)\end{array}$ & $\begin{array}{c}\text { Model IIB } \\
(a \neq b)\end{array}$ & SW [10] & $\begin{array}{c}\text { QCD } \\
\text { results [74] }\end{array}$ \\
\hline$F_{s_{0}}^{1 / 2}$ & 532.7 & 533.2 & 470.4 & 426.6 & 420 & 425.3 \\
$F_{s_{1}}^{1 / 2}$ & 779.7 & 780.1 & 561.1 & 465.7 & 499 & \\
$F_{s_{2}}^{1 / 2}$ & 582.5 & 582.5 & 595.8 & 544.5 & 552 & \\
\hline \hline
\end{tabular}



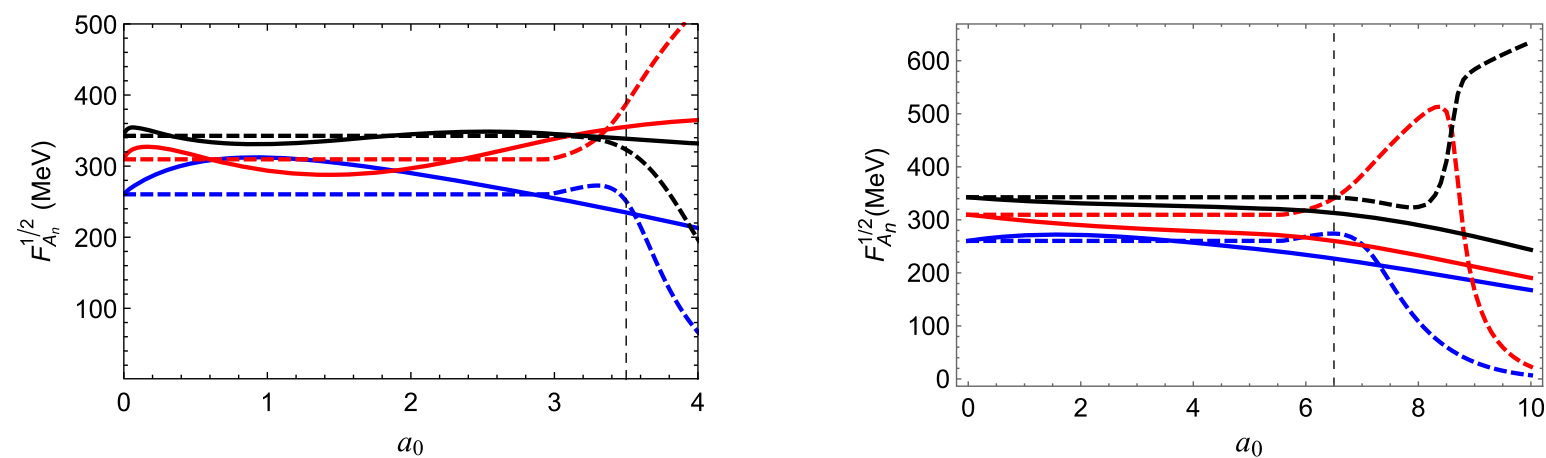

FIG. 18. Left: decay constants of axial-vector mesons as functions of $a_{0}$ for models of type I, where solid lines represent model IA $(\lambda=160)$, while dashed lines represent model IB $(\lambda=380)$. Right: decay constants of axial-vector mesons as functions of $a_{0}$ for models of type II, where solid lines represent model IIA $(\lambda=60)$, while dashed lines represent model IIB $(\lambda=413)$. We obtained these results for $m_{q}=3.63 \times 10^{-4} \mathrm{MeV}$ in all the models (near the chiral limit).
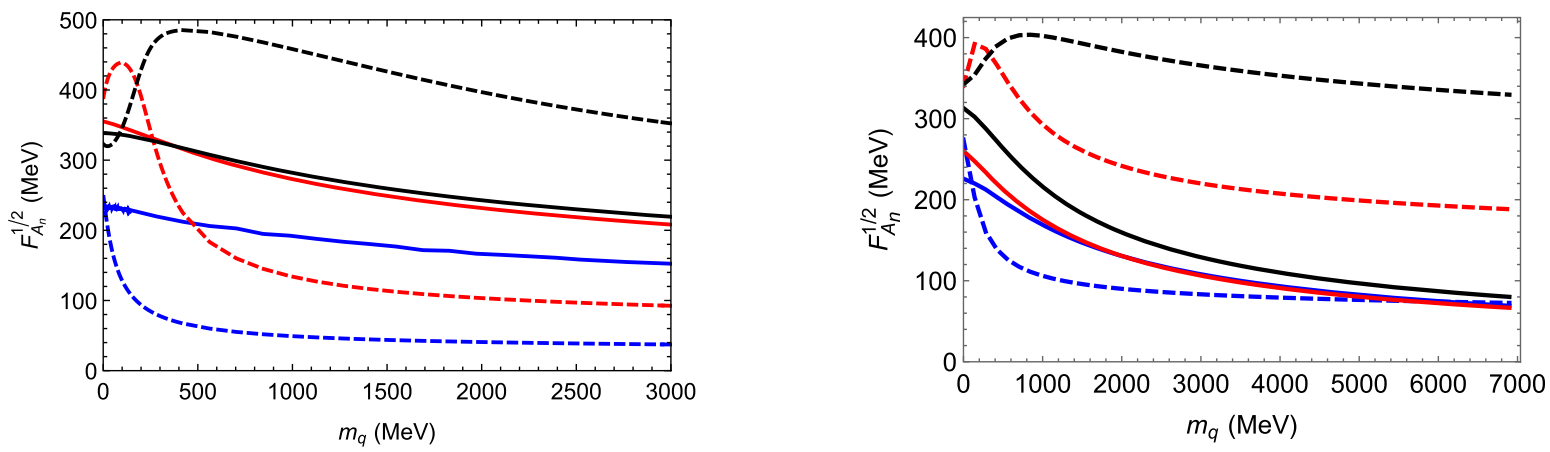

FIG. 19. Left: decay constants of axial-vector mesons as functions of $m_{q}$ in models of type I with $a_{0}=3.5$, where solid lines represent the results for model IA $(\lambda=160)$, while dashed lines represent results for model IB $(\lambda=380)$. Right: decay constants of axial-vector mesons as functions of $m_{q}$ in models of type II with $a_{0}=6.5$, where solid lines represent the results for model IIA ( $\left.\lambda=60\right)$, while dashed lines represent results for model IIB $(\lambda=413)$.

$$
\int_{\epsilon}^{\infty} d z \frac{e^{A_{s}-b}}{\beta(z)}\left(\partial_{z} \varphi_{m}\right)\left(\partial_{z} \varphi_{n}\right)=\frac{\delta_{m n}}{m_{\pi_{n}}^{2}}
$$

$$
f_{\pi_{n}}=-\left.\lim _{\epsilon \rightarrow 0} \frac{e^{A_{s}-b}}{g_{5}} \partial_{z} \varphi_{n}(z)\right|_{z=\epsilon}
$$

We follow in this work the prescription for calculating decay constants for the pion and their resonances developed in Refs. [23,51]. Details on the derivation are given in Appendix C. The decay constants of pseudoscalar mesons are calculated using the following holographic dictionary, cf. Eq. (C7):

Considering the UV expansion of the field $\varphi_{n}(z)$, which is given by $\varphi_{n}=-N_{\pi_{n}}\left(z^{2}+\cdots\right)$, where $N_{\pi_{n}}$ is the normalization constant obtained from (65), and plugging this expression into (66), we get the result

TABLE IX. Decay constants of axial-vector mesons (in $\mathrm{MeV}$ ) obtained in models of type I and II, compared against the result obtained in the linear soft wall model [10] and experimental results of [75]. These results were obtained for the set of parameters displayed in Table II, and the quark masses were fixed as $m_{q}=9 \mathrm{MeV}$, $m_{q}=4.7 \mathrm{MeV}, m_{q}=9.8 \mathrm{MeV}$, and $m_{q}=26.8 \mathrm{MeV}$ for models of type IA, IB, IIA, and IIB, respectively.

\begin{tabular}{lcccccc}
\hline \hline & $\begin{array}{c}\text { Model IA } \\
(a=b)\end{array}$ & $\begin{array}{c}\text { Model IB } \\
(a \neq b)\end{array}$ & $\begin{array}{c}\text { Model IIA } \\
(a=b)\end{array}$ & $\begin{array}{c}\text { Model IIB } \\
(a \neq b)\end{array}$ & SW [10] & $\begin{array}{c}\text { Experimental } \\
\left(F_{A^{c}}=f_{a_{1}} / \sqrt{2}\right)[75]\end{array}$ \\
\hline$F_{A_{0}}^{1 / 2}$ & 141.82 & 241 & 226.07 & 224.24 & 261 & $433 \pm 13$ \\
$F_{A_{1}}^{1 / 2}$ & 324.10 & 395 & 257.12 & 386.50 & & \\
$F_{A_{2}}^{1 / 2}$ & 295.17 & 322 & 310.91 & 348.54 & & \\
\hline \hline
\end{tabular}



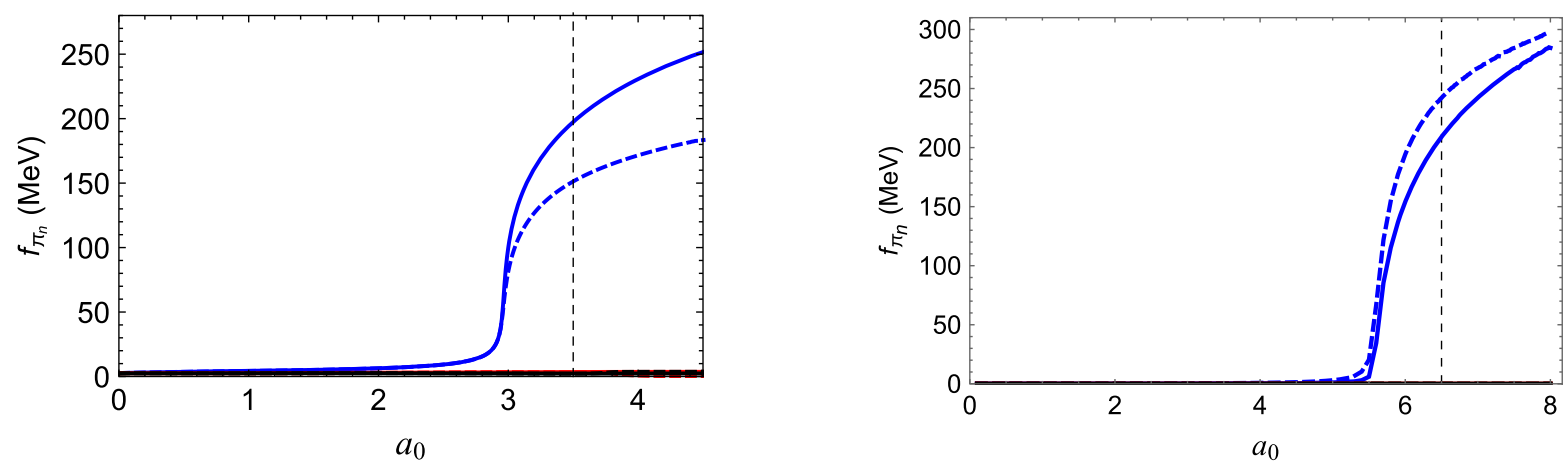

FIG. 20. Left: the decay constants of the pseudoscalar mesons as a function of $a_{0}$ for models of type I, where solid lines represent model IA $(\lambda=160)$, while dashed lines represent model IB $(\lambda=380)$. Right: the decay constants of the pseudoscalar mesons as a function of $a_{0}$ for models of type II, where solid lines represent model IIA $(\lambda=60)$, while dashed lines represent model IIB $(\lambda=413)$. We get these results for $m_{q}=3.63 \times 10^{-4} \mathrm{MeV}$ in both models.

$$
f_{\pi_{n}}=\frac{2}{g_{5}} N_{\pi_{n}}
$$

Once again, the problem of finding decay constants has been reduced to the calculation of normalization constants. Our numerical results for the decay constants in models of type I as functions of $a_{0}$ are displayed on the left panel of Fig. 20, where solid lines (dashed lines) represent results for model IA (IB). Similarly, the results in models of type II are displayed on the right panel of Fig. 20, where solid lines (dashed lines) represent results for model IIA (IIB). All these results were obtained fixing the quark mass as $m_{q}=$ $3.63 \times 10^{-4} \mathrm{MeV}$ (near the chiral limit).

Note that the decay constants of the excited states in all models are close to zero for $a_{0}<a_{0}^{c}$. This is consistent with the interpretation of these excited states in terms of pion resonances and is in qualitative agreement with the results obtained in Ref. [51], where no Nambu-Goldstone boson was found. Note, however, that the decay constant of the ground state starts to grow up for values close to the critical value $a_{0} \sim a_{0}^{c}$, meaning that it becomes finite for $a_{0} \geq a_{0}^{c}$. This is true for models I and II. These results support our

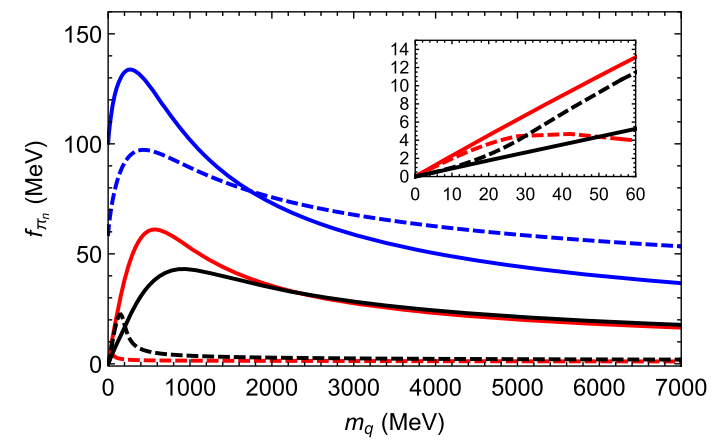

choice of $a_{0}=3.5$ for models of type I and $a_{0}=6.5$ for models of type II.

The decay constants of pseudoscalar mesons in models of type I, with $a_{0}=3.5$, as functions of the quark mass, are displayed on the left panel of Fig. 21, where results for model IA (IB) are represented by solid (dashed) lines. As expected, the decay constant of the ground state is finite in the chiral limit, which supports our conclusion that the ground state is the pion. Note also that the decay constants of the resonances are zero in this limit. This result was previously observed in the holographic hard wall model [23]. We observe from the figure that the decay constants are nonmonotonic functions of the quark mass, growing in the regime of light quarks and then decreasing in the regime of heavy quarks. We also observe that the hierarchy between decay constants changes to $f_{\pi_{0}}>$ $f_{\pi_{1}}>f_{\pi_{2}}$ in the regime of light quarks and becomes $f_{\pi_{0}}>$ $f_{\pi_{2}}>f_{\pi_{1}}$ in the regime of heavy quarks. The results obtained for the resonances are in qualitative agreement with the results obtained in Ref. [51]. The results obtained for models of type II, displayed on the right panel of Fig. 21, are qualitatively similar to the results obtained in models of type I.

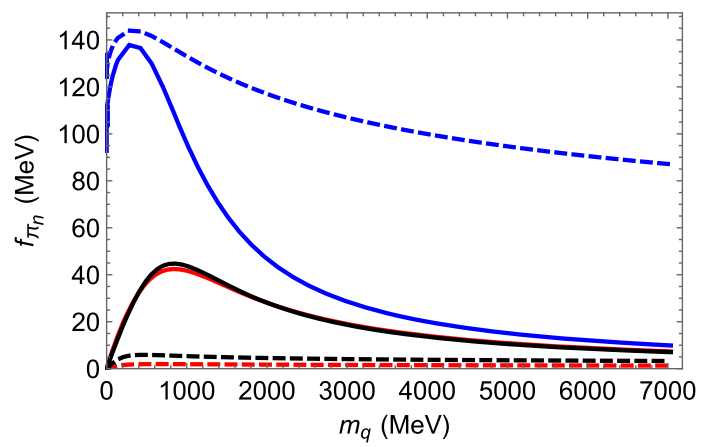

FIG. 21. Left: decay constants of pseudoscalar mesons as functions of $m_{q}$, where solid lines represent the results for model IA $(\lambda=160)$, while dashed lines represent results for model IB $(\lambda=380)$. Right: decay constants of pseudoscalar mesons as functions of $m_{q}$, where solid lines represent the results for model IIA $(\lambda=60)$, while dashed lines represent results for model IIB $(\lambda=413)$. 
TABLE X. Decay constants (in MeV) obtained in models of type I and models of type II for the set of parameters given in Table II, compared against the experimental results of the PDG [71]. The quark masses were fixed as $m_{q}=9 \mathrm{MeV}, m_{q}=4.7 \mathrm{MeV}, m_{q}=9.8 \mathrm{MeV}$, and $m_{q}=26.8 \mathrm{MeV}$ for models of type IA, IB, IIA, and IIB, respectively.

\begin{tabular}{lccccc}
\hline \hline & $\begin{array}{c}\text { Model IA } \\
(a=b)\end{array}$ & $\begin{array}{c}\text { Model IB } \\
(a \neq b)\end{array}$ & $\begin{array}{c}\text { Model IIA } \\
(a=b)\end{array}$ & $\begin{array}{c}\text { Model IIB } \\
(a \neq b)\end{array}$ & $\begin{array}{c}\text { Experimental } \\
\left(f_{\pi^{+}} / \sqrt{2}\right)[71]\end{array}$ \\
\hline$f_{\pi_{0}}$ & 104.3 & 60.9 & 118.3 & 138.68 & $92.1 \pm 0.8$ \\
$f_{\pi_{1}}$ & 2.05 & 0.95 & 3.94 & 1.04 & 2.97 \\
$f_{\pi_{2}}$ & 0.79 & 0.42 & 3.37 & \\
\hline \hline
\end{tabular}

So far, we have observed that the decay constant of the ground state can be related to the pion. To support this statement, we need the pion arising in the spectrum, as in fact we observed in Table VI. Having fixed the model parameters in Table II, we calculate the corresponding values of the pseudoscalar meson decay constants and display the results in Table $\mathrm{X}$. The quark masses were fixed as $m_{q}=9 \mathrm{MeV}, m_{q}=4.7 \mathrm{MeV}, m_{q}=9.8 \mathrm{MeV}$, and $m_{q}=26.8 \mathrm{MeV}$ for models of type IA, IB, IIA, and IIB, respectively.

\section{E. GOR relation}

We have identified the lightest sate arising in the pseudoscalar sector with the pion. As a final check, we show in this section that it satisfies the GOR relation. In the following analysis, we follow the procedure implemented in Ref. [14], adapted to the holographic model we are working with. The idea is to use the normalization condition (65) together with the definition of the decay constant (66). It is worth mentioning that the functions $a(\Phi)$ and $b(\Phi)$ are subleading close to the boundary, where the leading term is the warp factor $A_{s}=-\ln z$. Thus, we may rewrite (65) in the form (setting $m=n$ )

$g_{5}^{2} m_{\pi_{n}}^{2} \int_{\epsilon}^{\infty} d z \frac{e^{-A_{s}-b}}{\beta(z)}\left(\frac{e^{A_{s}}}{g_{5}} \partial_{z} \varphi_{n}\right)\left(\frac{e^{A_{s}}}{g_{5}} \partial_{z} \varphi_{n}\right)=1$

or

$m_{\pi_{n}}^{2} \int_{\epsilon}^{\infty} d z \frac{e^{-3 A_{s}-b}}{v^{2}(z)}\left(e^{a-b}\right)\left(\frac{e^{A_{s}}}{g_{5}} \partial_{z} \varphi_{n}\right)\left(\frac{e^{A_{s}}}{g_{5}} \partial_{z} \varphi_{n}\right)=1$,

where $\beta$ was defined in (37). Near the chiral limit, i.e., $m_{q} \rightarrow 0$, it can be shown that the term $e^{-3 A_{s}-b} / v^{2}(z)$ in the integral becomes highly peaked near the boundary $z=\epsilon$. If the terms in parentheses are nonzero in that limit, they can be moved outside of the integral. This is true only for the ground state because it is the only state that has a nonzero decay constant in the chiral limit; see Eq. (66) and Fig. 21. Then, for the ground state, we find the following result near the chiral limit:

$$
f_{\pi_{0}}^{2} m_{\pi_{0}}^{2} \int_{\epsilon}^{\infty} \frac{e^{-3 A_{s}-b}}{v^{2}(z)} d z=1
$$

Near the chiral limit, the integrand $e^{-3 A_{s}-b} / v^{2}(z)$ has its relevant contribution close to the boundary, i.e., $e^{-3 A_{s}-b} / v^{2}(z) \sim z /\left(c_{1}+c_{3} z^{2}\right)^{2}$. Hence, the integral provides the result

$$
\int_{\epsilon}^{\infty} \frac{e^{-3 A_{s}-b}}{v^{2}(z)} d z \approx \frac{1}{2 c_{1} c_{3}} .
$$

This result is valid only for small $c_{1}$. Then, plugging (71) in (70), and considering that $c_{1}=m_{q} \zeta$ and $c_{3}=\Sigma /(2 \zeta)$, we get the GOR relation

$$
f_{\pi_{0}}^{2} m_{\pi_{0}}^{2}=m_{q} \Sigma .
$$

One may rewrite the last equation using the relation $\Sigma=2 \sigma$, where $\sigma$ is the up and down quark condensates $\langle\bar{u} u\rangle=\langle\bar{d} d\rangle=\sigma$,

$$
f_{\pi_{0}}^{2} m_{\pi_{0}}^{2}=2 m_{q} \sigma
$$

To support the last procedure, we calculated the GOR relation numerically. Our results are displayed in Fig. 22. As can be seen, the ground state satisfies the GOR relation in the region of light quarks.

To understand better the approximation we have done in the integral (71), we refer the reader to Appendix G, where we discuss the derivation of the GOR relation using a toy model.

\section{CONCLUSIONS}

In this paper, we have extended the original soft wall model, considering a Higgs potential for the tachyonic field and nonminimal dilaton couplings $\exp (a(\Phi))$ and $\exp (b(\Phi))$ for the tachyonic and gauge field, respectively. As in the original soft wall model, the 5D metric was fixed as the AdS spacetime in Poincare coordinates, and the dilaton $\Phi$ was fixed to be quadratic in the radial coordinate $z$. In order to guarantee good properties for the meson spectrum, such as approximate linear behavior with the 

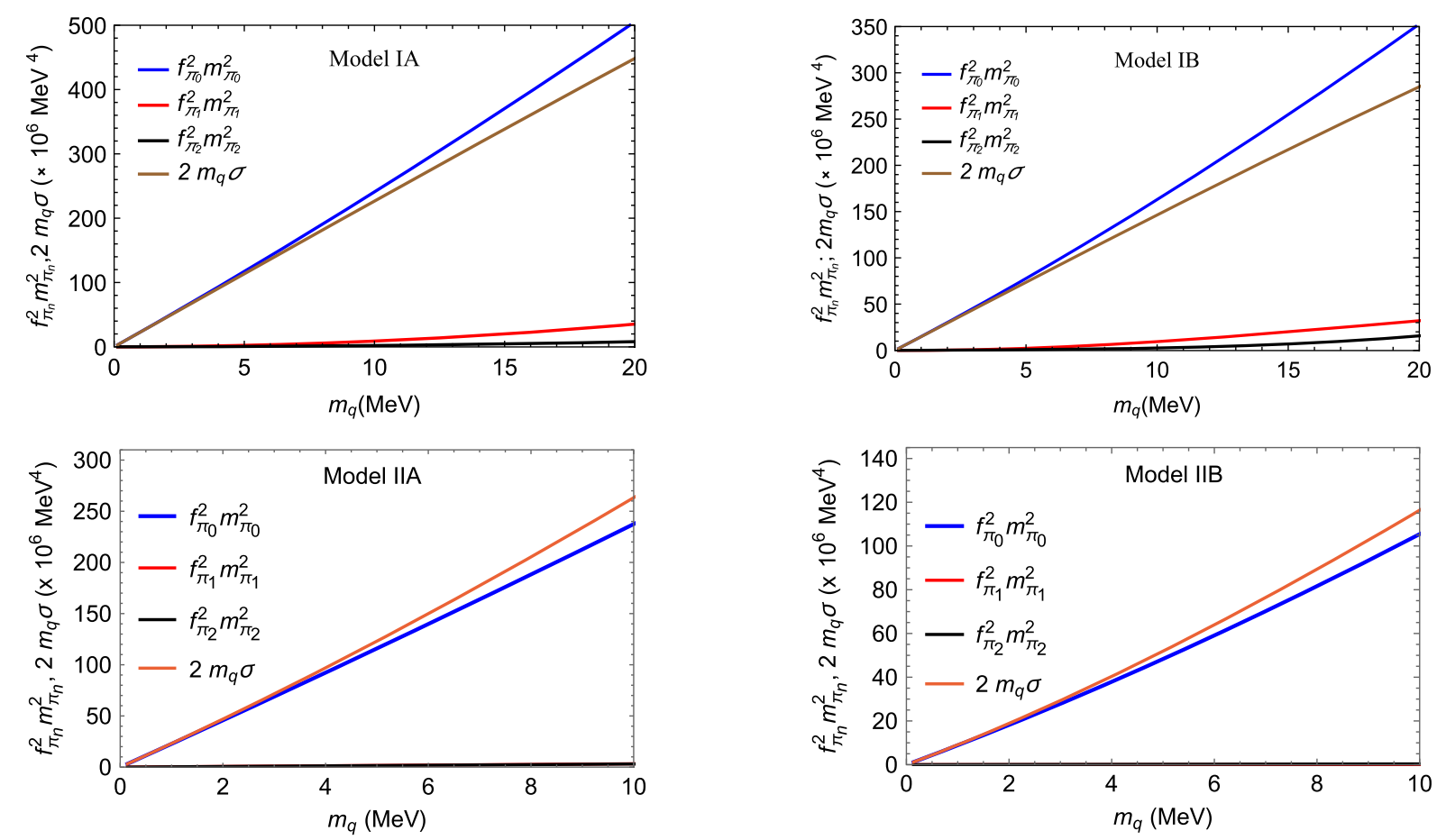

FIG. 22. The product $f_{\pi_{n}}^{2} m_{\pi_{n}}^{2}$ and $2 m_{q} \sigma$ as a function of the quark mass for models of type I (top panel) and models of type II (bottom panel). As can be seen, the GOR relation arises for the ground state in the regime of light quarks.

excitation number and absence of spurious massless modes, we imposed that at large $\Phi$ (far from the boundary), the nonminimal couplings reduce to the minimal case; i.e., $a(\Phi)$ and $b(\Phi)$ reduce to $\Phi$.

Our work was inspired by previous works on soft wall models such as Refs. $[13,19,52,53]$ that realized that considering an effective dilaton field, which in our notation corresponds to a particular choice for the nonminimal couplings $a(\Phi)=b(\Phi)=\tilde{\Phi}$, that is negative in some region, it is possible to incorporate spontaneous chiral symmetry. In order to avoid the massless mode found in Ref. [54] and instabilities in the background, we considered dilaton couplings $a(\Phi)$ and $b(\Phi)$ that satisfy the IR constraint $a(\Phi)=b(\Phi)=\Phi$. To simplify the analysis, we considered two possibilities for the dilaton coupling $b(\Phi)$, namely, $b(\Phi)=a(\Phi)$ (models of type A) and $b(\Phi)=\Phi$ (models of type B). For the dilaton coupling $a(\Phi)$, we considered two interpolations, namely, $a_{\mathrm{I}}(\Phi)$ (models of type I) and $a_{\mathrm{II}}(\Phi)$ (models of type II). The two interpolations $a_{\mathrm{I}}(\Phi)$ and $a_{\mathrm{II}}(\Phi)$, displayed in Fig. 1, share the feature that are nonmonotonic and become negative in some region. The main difference between them is that $a_{\mathrm{I}}(\Phi)$ reduces to $-a_{0} \Phi$ at small $\Phi$ (near the boundary), whereas $a_{\mathrm{II}}(\Phi)$ reduces to $\Phi$. The four different types of models IA, IB, IIA, and IIB are described in Table I. We have also stressed the fact that models IA and IIA could be embedded in a D-brane construction in string theory recasting the nonminimal couplings in terms of a global minimal coupling to an effective dilaton in a way similar to the original soft wall model [10]. We also pointed out that models IB and IIB do not seem to be consistent with a D-brane construction in perturbative string theory because the gauge fields and the tachyon field couple to the dilaton in different ways. Although it is not clear how these nonminimal couplings could arise from string theory (there may be inconsistencies at the quantum level), they are motivated by a bottom-up approach, such as that considered in AdS/ CMT [57], holographic QCD models at finite temperature and density [30,58-61], and holographic QCD models in the Veneziano limit $[48,62]$.

It is worth pointing out that soft wall models introduce an "artificial" confinement because the 5D metric is fixed to AdS spacetime and therefore the warp factor in the string frame does not have a minimum, required by confinement [76]. It turns out, however, that, taking into account the backreaction of the dilaton field into the 5D metric within the framework of Einstein-dilaton theories, the dilaton quadratic behavior predicted by the soft wall model at large $z$ remains intact; see, e.g., Refs. [29,32].

The nonminimal couplings introduced in this work brought a new parameter $a_{0}$ which controls the emergence of spontaneous chiral symmetry breaking, as shown in Fig. 4. We realized that the addition of a Higgs potential for the tachyonic field was not enough to induce spontaneous symmetry breaking, i.e., the emergence of the pion as a Nambu-Goldstone boson, as was previously shown in Ref. [51]. It seems to us that the reason behind the requirement of nonminimal couplings is due to the fact that the 
Higgs potential, usually introduced in 4D Minkowski spacetime to describe spontaneous symmetry breaking, has been extended to 5D AdS spacetime. The nonminimal dilaton couplings somehow compensate the deviations from the Mexican hat potential due to AdS spacetime. Moreover, we have verified that a scalar perturbation around the trivial solution $v(z)=0$ can be written in an AdS form where the effective 5D mass $m_{X}^{2}$ evolves with the radial direction $z$ violating the $\mathrm{BF}$ bound for some values of $z$. This $\mathrm{BF}$ violation was caused by the presence of the nonminimal dilaton couplings and signifies the instability of the trivial solution. This, in turn, indicates the presence of a nontrivial solution for $v(z)$ that is stable. This is the solution that we find in this work, and it is the gravity dual of a nonperturbative vacuum that breaks chiral symmetry spontaneously. We conclude that the addition of nonminimal couplings with an additional parameter is well justified in order to induce spontaneous chiral symmetry breaking in the chiral limit and the emergence of pions as Nambu-Goldstone bosons.

Once the nonminimal couplings were fixed, the tachyon profile was obtained numerically. We noticed a scaling symmetry for the tachyon solution that allowed us to find the solution $v(z)$ for any value of the Higgs coupling $\lambda$ in terms of the rescaled field $\tilde{v}(z)=\sqrt{\lambda} v(z)$. By solving the differential equations of the field perturbations, we were able to find the spectrum of vector, scalar, axial-vector, and pseudoscalar mesons. It turns out that the differential equation for the scalar sector depends only on $\tilde{v}(z)$, and therefore in the chiral limit, the scalar meson masses depend only on the parameter $a_{0}$ associated with the nonminimal coupling. Interestingly, the lightest scalar meson becomes massless exactly at the same critical value $a_{0}^{c}$ where the transition between explicit and spontaneous chiral symmetry breaking takes place. This strongly suggests that conformal symmetry was also spontaneously broken for that critical value. For $a_{0}>a_{0}^{c}$, we are in the regime of spontaneous chiral symmetry breaking, and the lightest scalar meson grows monotonically, as shown in Fig. 9. Considering these results, we fixed the parameter $a_{0}$ at some value above $a_{0}^{c}$ in order to guarantee the absence of a massless scalar meson and the presence of spontaneous chiral symmetry breaking. We fixed $a_{0}=3.5$ in models of type I and $a_{0}=6.5$ in models of type II. In turn, the parameter $\lambda$ of the Higgs potential was fixed separately for each model in order to guarantee axial-vector meson masses $m_{A_{n}}$ and pseudoscalar meson masses $m_{\pi^{n}}$ that do not present any crossing between the fundamental and excited states. Finally, the parameter $\phi_{\infty}$ of the dilaton field was fixed to the usual value used in soft wall models. The choices of parameters in the four different type of models considered in this work are summarized in Table II. For those particular parameter choices, we analyzed the evolution of meson masses with the quark mass in the scalar, axial, and pseudoscalar sector (the vectorial sector is independent of the quark mass in this framework). We found that all the meson masses grow monotonically with the quark mass and that, as we approach the chiral limit, massless modes emerge in the pseudoscalar sector, identified as the Nambu-Goldstone bosons of spontaneous chiral symmetry breaking. Finally, fixing the quark mass, we were able to compare our results for the meson masses against experimental data and previous results in soft wall models.

We also calculated the decay constants for all the mesons. We found a peculiar behavior for the decay constants of vector, scalar, and axial-vector mesons, changing the hierarchy in certain intervals of $a_{0}$. This peculiar behavior is almost absent in models of type II; see Figs. 15, 16, and 18. The decay constants of the pseudoscalar sector as a function of $a_{0}$ shows the emergence of the pion for $a_{0} \geq a_{0}^{c}$; see Fig. 20. For the fixed values of $a_{0}, \lambda$, and $\phi_{\infty}$ given in Table II, we investigated the evolution of the decay constants of axial-vector, scalar, and pseudoscalar mesons with the quark mass. We found that the hierarchy between fundamental and excited states change as the quark mass evolves from the near chiral limit to the heavy quark limit. In any case, all the decay constants decrease with the quark mass as we approach the heavy quark limit, which is in qualitative agreement with expectations from perturbative QCD. We showed that the emergence of the pions as Nambu-Goldstone bosons associated with spontaneous chiral symmetry breaking is supported by the decay constants in the pseudoscalar sector, obtained in Fig. 21. The results near the chiral limit are qualitatively similar to the results obtained in the hard-wall model [23]. As a check of consistency, we showed that our models satisfy the GOR relation; see Fig. 22. Finally, fixing the quark masses, we were able to compare our results for the decay constants against experimental data and previous results in the literature.

In conclusion, we have shown in this work that spontaneous chiral symmetry breaking can be described using a holographic setup that extends the original soft wall model by including a Higgs potential for the tachyon and nonminimal dilaton couplings for the tachyon and gauge fields. Our numerical results show no instabilities in the spectrum. We realized that an additional parameter was needed in order to control the transition between spontaneous and explicit chiral symmetry breaking. The nonminimal dilaton couplings can be justified by the fact that we are describing the dynamics of a Higgs potential for a scalar field (the tachyon) in a curved space (AdS). These nonminimal couplings, combined with the Higgs potential, allowed us to find solutions for the tachyonic field with a VEV coefficient that remains finite in the limit where the source coefficient goes to zero (chiral limit). It is possible that the requirement of nonminimal couplings for describing spontaneous chiral symmetry breaking is an artifact of soft wall models in the sense that the background is not obtained by solving the Einstein's equations. 
We will test this hypothesis investigating chiral symmetry breaking in backgrounds obtained by solving the Einsteindilaton equations; see, for instance, Refs. [29,31-33]. Since those backgrounds describe color confinement in a consistent way, it would be interesting to investigate the connection between confinement and spontaneous chiral symmetry breaking.

Further extensions of this project include finite temperature effects of mesons and quarks in a nonconformal plasma [77-81] as well as its relation to the spectrum of quasinormal modes. Backreaction effects of the tachyonic and gauge fields would also be an interesting problem in order to find the quark contribution to the thermodynamics and transport coefficients of a nonconformal plasma (for related work in the top-down approach, see Refs. [82,83]). We will address some of these problems in the future.

\section{ACKNOWLEDGMENTS}

The work of A. B.-B. is partially funded by Conselho Nacional de Desenvolvimento Científico e Tecnológico (CNPq, Brazil), Grants No. 306528/2018-5 and No. 434523/ 2018-6, and Coordenação de Aperfeiçoamento do Pessoal de Nível Superior (CAPES, Brazil), Finance Code 001. L. A. H. M. has financial support from Coordenação de Aperfeiçoamento do Pessoal de Nível Superior-Programa Nacional de Pós-Doutorado (PNPD/CAPES, Brazil). D. M. R. is supported by Grant No. 2021/01565-8, São Paulo Research Foundation (FAPESP).

\section{APPENDIX A: NUMERICAL ANALYSIS}

Here, we write details on the numerical procedure. We realized that the numerical results obtained solving the tachyon differential equation (14) can be split up in three regions depending on the value for the parameter $a_{0}$.

First, for $0 \leq a_{0}<a_{0}^{c}$, one may vary $\tilde{C}_{0}$ continuously in the interval $-\sqrt{6} \leq \tilde{C}_{0} \leq \sqrt{6}$. In this region, there is explicit chiral symmetry breaking because $\tilde{c}_{3}$ is zero in

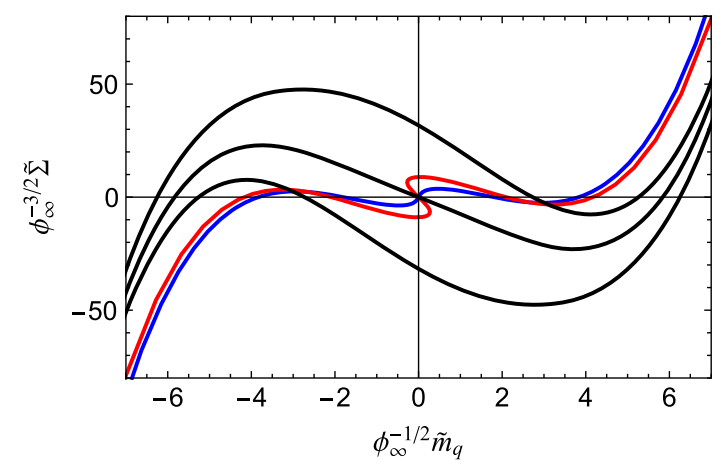

the chiral limit, i.e., $\tilde{m}_{q}=0$; note that $\tilde{m}_{q} \propto \tilde{C}_{0}$ in this region. Moreover, $a_{0}^{c}$ depends on the form for the interpolation function. Here, we work with models I and II. The numerical results for $\phi_{\infty}^{-3 / 2} \tilde{\Sigma}$ as a function of $\phi_{\infty}^{-1 / 2} \tilde{m}_{q}$ are displayed in Fig. 23 with blue lines for both models. As can be seen, the parameter $\phi_{\infty}^{-3 / 2} \tilde{\Sigma}$ goes to zero in the chiral limit for $a_{0}<a_{0}^{c}$.

Second, for $a_{0}^{c} \leq a_{0} \leq a_{0}^{\max }, \tilde{C}_{0}$ still vary in the interval $-\sqrt{6} \leq \tilde{C}_{0} \leq \sqrt{6}$. Nevertheless, there is spontaneous chiral symmetry breaking because $\phi_{\infty}^{-3 / 2} \tilde{\Sigma}$ is nonzero in the chiral limit. $a_{0}^{\max } \sim 5.954$ for models of type I. In this region, the parameter $a_{0}$ controls the value of the condensate. Our numerical results for models I and II are displayed in Fig. 23 with red lines, and left panel shows $\phi_{\infty}^{-3 / 2} \tilde{\Sigma}$ as a function of $\phi_{\infty}^{-1 / 2} \tilde{m}_{q}$ for light quarks. However, as can be seen in the right panel of this figure, $\phi_{\infty}^{-3 / 2} \tilde{\Sigma}$ becomes negative in the intermediate region. Maybe this behavior is suggesting that the model with negative dilaton in the UV is not enough to describe a monotonic increasing function for $\phi_{\infty}^{-3 / 2} \tilde{\Sigma}$ expected in real world QCD. In turn, models of type II provide improved results compared against the results obtained in models of type I, as can be seen in the right panel of Fig. 23. This means that the negative dilaton in the intermediate region is enough to guarantee a monotonic increasing function for $\phi_{\infty}^{-3 / 2} \tilde{\Sigma}$ as a function of $\phi_{\infty}^{-1 / 2} \tilde{m}_{q}$.

Third, for $a_{0}>a_{0}^{\max }, \tilde{C}_{0}$ is constrained to take values only close to the minimum of the Higgs potential $\tilde{C}_{0}<\sqrt{6}-\delta_{1}$, $\tilde{C}_{0}>-\sqrt{6}+\delta_{1}$, and close to the trivial solution $-\delta_{2} \leq \tilde{C}_{0}=0 \leq \delta_{2}$, where $\delta_{1}$ and $\delta_{2}$ are small numbers. This means that the solution splits up into three branches. The numerical results for models of type I, where $a_{0} \sim 5.954$, are displayed in the left panel of Fig. 23 with black lines. As can be seen, each branch corresponds to the solutions mentioned above. Note again that the results for models of type I show a negative condensate in the

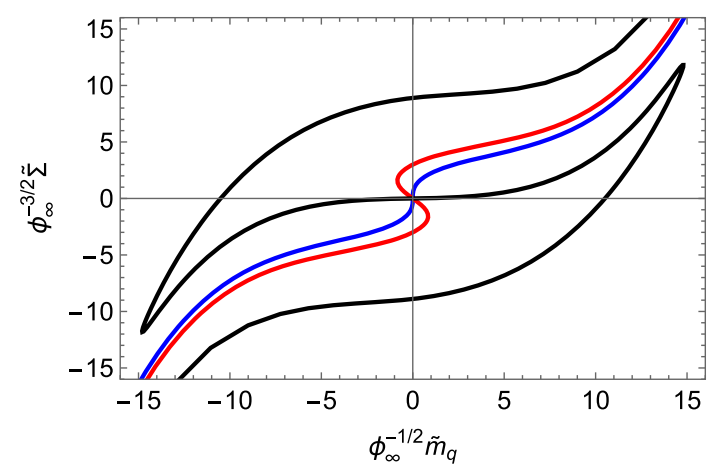

FIG. 23. Left: dimensionless condensate $\phi_{\infty}^{-3 / 2} \tilde{\Sigma}$ as a function of the dimensionless quark mass $\phi_{\infty}^{-1 / 2} \tilde{m}_{q}$ for models of type I with different values of $a_{0}: a_{0}=2.8$ (blue line), $a_{0}=3.5$ (red line), and $a_{0}=6$ (black lines). Right: dimensionless condensate $\phi_{\infty}^{-3 / 2} \tilde{\Sigma}$ as a function of the dimensionless quark mass $\phi_{\infty}^{-1 / 2} \tilde{m}_{q}$ for models of type II with different values of $a_{0}: a_{0}=5.6$ (blue line), $a_{0}=6.5$ (red line), and $a_{0}=10$ (black line). 

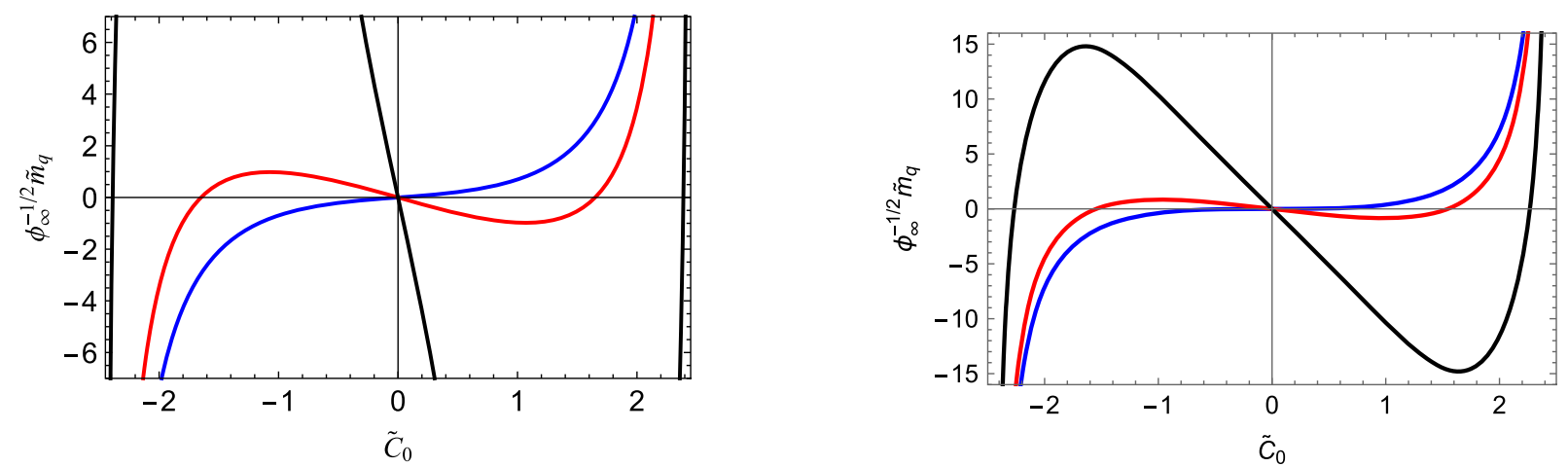

FIG. 24. Left: dimensionless quark mass $\phi_{\infty}^{-1 / 2} \tilde{m}_{q}$ as a function of $\tilde{C}_{0}$ for models of type I with different values of $a_{0}: a_{0}=2.8$ (blue line), $a_{0}=3.5$ (red line), and $a_{0}=6$ (black line). Right: dimensionless quark mass $\phi_{\infty}^{-1 / 2} \tilde{m}_{q}$ as a function of $\tilde{C}_{0}$ for models of type II with different values of $a_{0}: a_{0}=5.6$ (blue line), $a_{0}=6.5$ (red line), and $a_{0}=10$ (black line).

intermediate region. In turn, numerical results for models of type II are displayed in the right panel of this figure. Note also that the condensate $\phi_{\infty}^{-3 / 2} \tilde{\Sigma}$ is a monotonically increasing function of $\phi_{\infty}^{-1 / 2} \tilde{m}_{q}$; see the right panel.

On the other hand, a complementary analysis for models of type I shows the evolution of the dimensionless quark mass $\phi_{\infty}^{-1 / 2} \tilde{m}_{q}$ as a function of $\tilde{C}_{0}$ in the left panel of Fig. 24. As can be seen, $\tilde{C}_{0}$ is constrained to take values close to the minimum of the Higgs potential, as well as close to the trivial solution for $a_{0}>a_{0}^{\max }$. In turn, the right panel of this figure shows the results for models of type II.

Finally, we also get the dimensionless condensate $\phi_{\infty}^{-3 / 2} \tilde{\Sigma}$ as a function of $\tilde{C}_{0}$. Our numerical results for models of type I are displayed in the left panel of Fig. 25. As can be seen, $\tilde{C}_{0}$ is constrained to take values close to the minimum of the Higgs potential, as well as close to the trivial solution for $a_{0}>a_{0}^{\max }$. In turn, the right panel of this figure shows the corresponding results for models of type II.

\section{APPENDIX B: THE KALUZA-KLEIN EXPANSION}

The starting point is the $5 \mathrm{D}$ action

$$
\begin{aligned}
S_{2}= & -V_{4} \int d z\left\{e^{3 A_{s}-a}\left[\frac{1}{2}\left(\partial_{\hat{m}} S\right)^{2}+\frac{1}{2} e^{2 A_{s}} \frac{d^{2} U}{d v^{2}} S^{2}\right]\right. \\
& \left.+\frac{1}{g_{5}^{2}} e^{A_{s}-b}\left[\frac{1}{4} v_{\hat{m} \hat{n}}^{c}+\frac{1}{4} a_{\hat{m} \hat{n}}^{c}{ }^{2}+\frac{\beta}{2}\left(\partial_{\hat{m}} \pi^{c}-A_{\hat{m}}^{c}\right)^{2}\right]\right\},
\end{aligned}
$$

that dictates the dynamics of the field perturbations. We decompose the 5D gauge fields $V_{\hat{m}}=\left(V_{z}, V_{\hat{\mu}}\right), A_{\hat{m}}=$ $\left(A_{z}, A_{\hat{\mu}}\right)$ and also the derivatives $\partial_{\hat{m}}=\left(\partial_{z}, \partial_{\hat{\mu}}\right)$. The vectorial gauge symmetry and the residual axial gauge symmetry allow us to set $V_{z}$ and $A_{z}$ to zero, respectively. Then, the action (B1) takes the form

$$
\begin{aligned}
S_{2}= & -V_{4} \int d z\left\{e^{3 A_{s}-a}\left[\frac{1}{2}\left(\partial_{\hat{\mu}} S\right)^{2}+\frac{1}{2}\left(\partial_{z} S\right)^{2}+\frac{1}{2} e^{2 A_{s}} \frac{d^{2} U}{d v^{2}} S^{2}\right]\right. \\
& +\frac{1}{g_{5}^{2}} e^{A_{s}-b}\left[\frac{1}{4} v_{\hat{\mu} \hat{\nu}}^{c}+\frac{1}{2}\left(\partial_{z} V_{\hat{\mu}}^{c}\right)^{2}+\frac{1}{4} a_{\hat{\mu} \hat{\nu}}^{c}+\frac{1}{2}\left(\partial_{z} A_{\hat{\mu}}^{c}\right)^{2}\right. \\
& \left.\left.+\frac{\beta}{2}\left(\partial_{\hat{\mu}} \pi^{c}-A_{\hat{\mu}}^{c}\right)^{2}+\frac{\beta}{2}\left(\partial_{z} \pi^{c}\right)^{2}\right]\right\} .
\end{aligned}
$$

Implementing the Lorentz decomposition

$$
V_{\hat{\mu}, a}=V_{\hat{\mu}, a}^{\perp}+\partial_{\hat{\mu}} \xi^{a}, \quad A_{\hat{\mu}, a}=A_{\hat{\mu}, a}^{\perp}+\partial_{\hat{\mu}} \varphi^{a},
$$

the action (B2) becomes

$$
\begin{aligned}
S_{2}= & -V_{4} \int d z\left\{e^{3 A_{s}-a}\left[\frac{1}{2}\left(\partial_{\hat{\mu}} S\right)^{2}+\frac{1}{2}\left(\partial_{z} S\right)^{2}+\frac{1}{2} e^{2 A_{s}} \frac{d^{2} U}{d v^{2}} S^{2}\right]\right. \\
& +\frac{1}{g_{5}^{2}} e^{A_{s}-b}\left[\frac{1}{4} v_{\hat{\mu} \hat{\nu}}^{\perp, c 2}+\frac{1}{2}\left(\partial_{z} V_{\hat{\mu}}^{\perp, c}+\partial_{\hat{\mu}} \partial_{z} \xi c\right)^{2}\right. \\
& +\frac{1}{4} a_{\hat{\mu} \hat{\nu}}^{\perp, c 2}+\frac{1}{2}\left(\partial_{z} A_{\hat{\mu}}^{\perp, c}+\partial_{\hat{\mu}} \partial_{z} \varphi^{c}\right)^{2} \\
& \left.\left.+\frac{\beta}{2}\left(\partial_{\hat{\mu}} \pi^{c}-\partial_{\hat{\mu}} \varphi^{c}-A_{\hat{\mu}}^{\perp, c}\right)^{2}+\frac{\beta}{2}\left(\partial_{z} \pi^{c}\right)^{2}\right]\right\} .
\end{aligned}
$$

The fields $\xi^{c}$ can be set consistently to zero, and we do that in the following. ${ }^{3}$

\footnotetext{
${ }^{3}$ Nonzero $\xi^{c}$ would correspond to scalar mesons in the $\mathbf{3}$ representation with zero mass, not expected in QCD.
} 

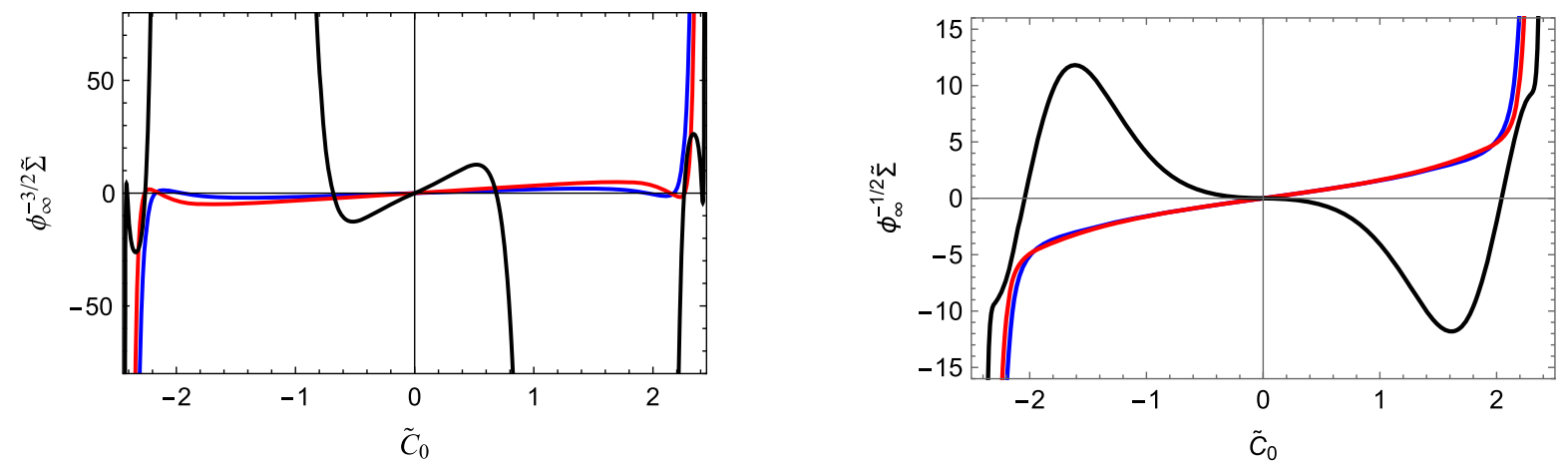

FIG. 25. Left: dimensionless condensate $\phi_{\infty}^{-3 / 2} \tilde{\Sigma}$ as a function of $\tilde{C}_{0}$ for models of type I with different values of $a_{0}: a_{0}=2.8$ (blue line), $a_{0}=3.5$ (red line), and $a_{0}=6$ (black lines). Right: dimensionless condensate $\phi_{\infty}^{-3 / 2} \tilde{\Sigma}$ as a function of $\tilde{C}_{0}$ for models of type II with different values of $a_{0}: a_{0}=5.6$ (blue line), $a_{0}=6.5$ (red line), and $a_{0}=10$ (black lines).

Now, we introduce the Kaluza-Klein expansions

$$
\begin{aligned}
V_{\hat{\mu}}^{\perp, c}(x, z) & =g_{5} v_{n}^{c}(z) \hat{V}_{\hat{\mu}}^{c, n}(x), \\
A_{\hat{\mu}}^{\perp, c}(x, z) & =g_{5} a_{n}^{c}(z) \hat{A}_{\hat{\mu}}^{c, n}(x), \\
S(x, z) & =s_{n}(z) \hat{S}_{n}(x), \\
\varphi^{c}(x, z) & =g_{5} \varphi_{n}^{c}(z) \hat{\Pi}_{n}^{c}(x), \\
\pi^{c}(x, z) & =g_{5} \pi_{n}^{c}(z) \hat{\Pi}_{n}^{c}(x),
\end{aligned}
$$

where the sum $\sum_{n=0}^{\infty}$ is implicit. Plugging (B5) into (B4), with $\xi^{c}=0$, we arrive at the $4 \mathrm{D}$ action $S_{2}=-\int d^{4} x \mathcal{L}_{2}$ with

$$
\begin{aligned}
\mathcal{L}_{2}= & \frac{1}{2} \Delta_{m n}^{(S)}\left(\partial_{\hat{\mu}} \hat{S}_{m}\right)\left(\partial^{\hat{\mu}} \hat{S}_{n}\right)+\frac{1}{2} M_{m n}^{(S)} \hat{S}_{m} \hat{S}_{n} \\
& +\frac{1}{4} \Delta_{m n, c d}^{(V)} \hat{v}_{\hat{\mu} \nu}^{c, m} \hat{v}_{d, n}^{\hat{\mu} \hat{\nu}}+\frac{1}{2} M_{m n, c d}^{(V)} \hat{V}_{\hat{\mu}}^{c, m} \hat{V}_{d, n}^{\hat{\mu}} \\
& +\frac{1}{4} \Delta_{m n, c d}^{(A)} \hat{a}_{\hat{\mu} \nu}^{c, m} \hat{a}_{d, n}^{\hat{\mu} \hat{\nu}}+\frac{1}{2} M_{m n, c d}^{(A)} \hat{A}_{\hat{\mu}}^{c, m} \hat{A}_{d, n}^{\hat{\mu}} \\
& +\frac{1}{2} \Delta_{m n, c d}^{(\Pi)}\left(\partial_{\hat{\mu}} \hat{\Pi}_{m}^{c}\right)\left(\partial^{\hat{\mu}} \hat{\Pi}_{n}^{d}\right)+\frac{1}{2} M_{m n, c d}^{(\Pi)} \hat{\Pi}_{m}^{c} \hat{\Pi}_{n}^{d} \\
& +G_{m n, c d}^{(A \Pi)} \hat{A}_{\hat{\mu}}^{c, m} \partial^{\hat{\mu} \hat{\Pi}^{d, n}}
\end{aligned}
$$

and we have defined the $4 \mathrm{D}$ coefficients

$$
\begin{aligned}
\Delta_{m n}^{(S)} & =\int d z e^{3 A_{s}-a} s_{m} s_{n}, \quad M_{m n}^{(S)}=\int d z e^{3 A_{s}-a}\left[\left(\partial_{z} s_{m}\right)\left(\partial_{z} s_{n}\right)+e^{2 A_{s}} \frac{d^{2} U}{d v^{2}} s_{m} s_{n}\right], \\
\Delta_{m n, c d}^{(V)} & =\delta^{c d} \int d z e^{A_{s}-b} v_{m}^{c} v_{n}^{d}, \quad M_{m n, c d}^{(V)}=\delta^{c d} \int d z e^{A_{s}-b}\left(\partial_{z} v_{m}^{c}\right)\left(\partial_{z} v_{n}^{d}\right), \\
\Delta_{m n, c d}^{(A)} & =\delta^{c d} \int d z e^{A_{s}-b} a_{m}^{c} a_{n}^{d}, \quad M_{m n, c d}^{(A)}=\delta^{c d} \int d z e^{A_{s}-b}\left[\left(\partial_{z} a_{m}^{c}\right)\left(\partial_{z} a_{n}^{d}\right)+\beta a_{m}^{c} a_{n}^{d}\right], \\
\Delta_{m n, c d}^{(\Pi)} & =\delta^{c d} \int d z e^{A_{s}-b}\left[\left(\partial_{z} \varphi_{m}^{c}\right)\left(\partial_{z} \varphi_{n}^{d}\right)+\beta\left(\pi_{m}^{c}-\varphi_{m}^{c}\right)\left(\pi_{n}^{d}-\varphi_{n}^{d}\right)\right], \\
M_{m n, c d}^{(\Pi)} & =\delta^{c d} \int d z e^{A_{s}-b} \beta\left(\partial_{z} \pi_{m}^{c}\right)\left(\partial_{z} \pi_{n}^{d}\right), \\
G_{m n, c d}^{(A \Pi)} & =\delta^{c d} \int d z e^{A_{s}-b}\left[\left(\partial_{z} a_{m}^{c}\right)\left(\partial_{z} \varphi_{n}^{d}\right)-\beta a_{m}^{c}\left(\pi_{n}^{d}-\varphi_{n}^{d}\right)\right] .
\end{aligned}
$$

The Lagrangian (B6) becomes the standard Lagrangian for mesons if we impose the conditions

$$
\Delta_{m n}^{(S)}=\delta_{m n}, \quad \Delta_{m n, c d}^{(V)}=\Delta_{m n, c d}^{(A)}=\Delta_{m n, c d}^{(\Pi)}=\delta^{c d} \delta_{m n}
$$

for the normalization coefficients and conditions 


$$
\begin{array}{rlrl}
M_{m n}^{(S)} & =m_{s_{n}}^{2} \delta_{m n}, & M_{m n, c d}^{(V)}=m_{v_{n}}^{2} \delta^{c d} \delta_{m n} \\
M_{m n, c d}^{(A)} & =m_{a_{n}}^{2} \delta^{c d} \delta_{m n}, & & M_{m n, c d}^{(\Pi)}=m_{\pi_{n}}^{2} \delta^{c d} \delta_{m n} \\
G_{m n, c d}^{(A \Pi)} & =0
\end{array}
$$

for the mass coefficients and couplings. In fact, the conditions (B9) are automatically satisfied if the Kaluza-Klein modes satisfy the following differential equations,

$$
\begin{aligned}
& \left\{\left[\partial_{z}+3 A_{s}^{\prime}-a^{\prime}\right] \partial_{z}-e^{2 A_{s}} \frac{d^{2} U}{d v^{2}}\right\} s_{n}=-m_{s_{n}}^{2} s_{n} \quad \text { (scalar sector), } \\
& {\left[\partial_{z}+A_{s}^{\prime}-b^{\prime}\right] \partial_{z} v_{n}^{c}=-m_{v_{n}}^{2} v_{n}^{c} \quad \text { (vectorial sector), }} \\
& \left\{\left[\partial_{z}+A_{s}^{\prime}-b^{\prime}\right] \partial_{z}-\beta\right\} a_{n}^{c}=-m_{a_{n}}^{2} a_{n}^{c} \quad \text { (axial sector) } \\
& \quad\left[\partial_{z}+A_{s}^{\prime}-b^{\prime}\right] \partial_{z} \varphi_{n}^{c}=-\beta\left(\pi_{n}^{c}-\varphi_{n}^{c}\right), \\
& \beta \partial_{z} \pi_{n}^{c}=m_{\pi_{n}}^{2} \partial_{z} \varphi_{n}^{c} \quad \text { (pseudoscalar sector) }
\end{aligned}
$$

and are normalized according to (B8). The differential equations (B10) satisfied by the Kaluza-Klein modes are compatible with the field equations (46)-(49) for the 5D fields. The main difference between those equations is that (B10) are on-shell conditions involving only the $z$ dependence of the 5D fields whereas (46)-(49) are on-shell conditions for the $z$ and $x$ dependence. We see that the Kaluza-Klein expansions allows us to consider the 4D fields in (B6) off shell.

Note that the normalization conditions for the scalar, vectorial, and axial sectors can be written as

$$
\begin{aligned}
\Delta_{m n}^{(S)} & =\int d z \psi_{s_{m}} \psi_{s_{n}}=\delta_{m n}, \\
\Delta_{m n}^{(V)} & =\int d z \psi_{v_{m}} \psi_{v_{n}}=\delta_{m n}, \\
\Delta_{m n}^{(A)} & =\int d z \psi_{a_{m}} \psi_{a_{n}}=\delta_{m n},
\end{aligned}
$$

where $\psi_{s_{n}}, \psi_{v_{n}}$, and $\psi_{a_{n}}$ are the wave functions associated with the Schrödinger problem in the scalar, vectorial, and axial sectors defined previously. For simplicity, we have omitted the flavor index in the vectorial and axial sector. We conclude that the Schrödinger problem in those sectors is well posed in the sense that the wave functions form an orthonormal basis.

\section{APPENDIX C: MESON OPERATORS AND DECAY CONSTANTS}

From (41), we find that the on-shell variation of $S_{2}$ can be written as

$$
\begin{aligned}
\delta S_{2}^{o-s}= & \int d^{4} x\left[e^{3 A_{s}-a} \partial_{z} S \delta S+\frac{1}{g_{5}^{2}} e^{A_{s}-b} v_{c}^{\hat{z} \hat{\mu}} \delta V_{\hat{\mu}}^{c}\right. \\
& \left.+\frac{1}{g_{5}^{2}} e^{A_{s}-b} a_{c}^{\hat{z} \hat{\mu}} \delta A_{\hat{\mu}}^{c}+\frac{1}{g_{5}^{2}} \beta e^{A_{s}-b}\left(\partial^{\hat{z}} \pi^{c}-A^{\hat{z}, c}\right) \delta \pi^{c}\right]_{z=\epsilon},
\end{aligned}
$$

where all the 5D fields satisfy the equations of motion. The 5D scalar field $S(x, z)$ behaves near the boundary as

$$
S(x, z)=S_{1}(x) z+T_{3}(x) z^{3} \ln z+S_{3}(x) z^{3}+\ldots,
$$

where $S_{1}(x)=\zeta^{-1} m_{q}(x)$ is the source coefficient. The $\mathrm{VEV}$ of the scalar operator responsible for creation of scalar mesons can be obtained from the holographic dictionary:

$$
\begin{aligned}
\langle\bar{q}(x) q(x)\rangle & =\frac{\delta S_{2}^{o-s}}{\delta m_{q}(x)}=\zeta \frac{\delta S_{2}^{o-s}}{\delta S_{1}(x)} \\
& =\zeta\left[z e^{3 A_{s}-a} \partial_{z} S\right]_{z=\epsilon}
\end{aligned}
$$

The vectorial and axial gauge fields behave near the boundary as

$$
\begin{aligned}
& V_{\hat{\mu}, c}(x, z)=V_{\hat{\mu}, c}^{(0)}(x)+W_{\hat{\mu}, c}^{(2)}(x) z^{2} \ln z+V_{\hat{\mu}, c}^{(2)}(x) z^{2}+\ldots, \\
& A_{\hat{\mu}, c}(x, z)=A_{\hat{\mu}, c}^{(0)}(x)+B_{\hat{\mu}, c}^{(2)}(x) z^{2} \ln z+A_{\hat{\mu}, c}^{(2)}(x) z^{2}+\ldots
\end{aligned}
$$

with $V_{\hat{\mu}, c}^{(0)}(x)$ and $A_{\hat{\mu}, c}^{(0)}(x)$ 4D external sources. The corresponding vectorial and axial currents are then given by 


$$
\begin{gathered}
\left\langle\bar{q}(x) \gamma^{\mu} T^{c} q(x)\right\rangle=\frac{\delta S_{2}^{o-s}}{\delta V_{\hat{\mu}, c}^{(0)}(x)}=\frac{1}{g_{5}^{2}}\left[e^{A_{s}-b} v_{c}^{\hat{z} \hat{\mu}}\right]_{z=\epsilon} \\
\left\langle\bar{q}(x) \gamma^{\mu} \gamma^{5} T^{c} q(x)\right\rangle=\frac{\delta S_{2}^{o-s}}{\delta A_{\hat{\mu}, c}^{(0)}(x)}=\frac{1}{g_{5}^{2}}\left[e^{A_{s}-b} a_{c}^{\hat{z} \hat{\mu}}\right]_{z=\epsilon} .
\end{gathered}
$$

Plugging the Kaluza-Klein expansions (B5) into (C3) and (C5), we obtain a mode expansion for the scalar operator and currents,

$$
\begin{aligned}
\langle\bar{q}(x) q(x)\rangle & =F_{s^{n}} \hat{S}_{n}(x), \\
\left\langle\bar{q}(x) \gamma^{\mu} T^{c} q(x)\right\rangle & =F_{v^{c, n}} \hat{V}_{\hat{\mu}}^{c, n}(x), \\
\left\langle\bar{q}(x) \gamma^{\mu} \gamma^{5} T^{c} q(x)\right\rangle & =F_{a^{c, n}} \hat{A}_{\hat{\mu}}^{c, n}(x)-f_{\pi^{c, n}} \partial^{\hat{\mu}} \hat{\pi}^{c, n}(x),
\end{aligned}
$$

where the sum $\sum_{n=0}^{\infty}$ is implicit and the Kaluza-Klein coefficients are given,

$$
\begin{aligned}
F_{s^{n}} & =\zeta\left[z e^{3 A_{s}-a} \partial_{z} s_{n}\right]_{z=\epsilon}, \\
F_{v^{c, n}} & =\frac{1}{g_{5}}\left[e^{A_{s}-b} \partial_{z} v_{c, n}\right]_{z=\epsilon}, \\
F_{a^{c, n}} & =\frac{1}{g_{5}}\left[e^{A_{s}-b} \partial_{z} a_{c, n}\right]_{z=\epsilon}, \\
f_{\pi^{c, n}} & =-\frac{1}{g_{5}}\left[e^{A_{s}-b} \partial_{z} \varphi_{c, n}\right]_{z=\epsilon} .
\end{aligned}
$$

The coefficients $F_{s^{n}}, F_{v^{c, n}}, F_{a^{c, n}}$, and $f_{\pi^{c, n}}$ are identified with the decay constants of the scalar, vectorial, axial, and pseudoscalar mesons.

\section{APPENDIX D: VECTOR AND SCALAR MESONS IN THE LINEAR SOFT WALL MODEL}

In the limit $a_{0} \rightarrow 0$ (minimal coupling), the differential equation for the vectorial mode $v^{n, c}$ in all models (IA, IB, IIA, and IIB) reduces to

$$
\left[\partial_{z}+A_{s}^{\prime}-\Phi^{\prime}\right] \partial_{z} v_{n}^{c}=-m_{v_{n}}^{2} v_{n}^{c}
$$

Since $A_{s}=-\ln z$ and $\Phi(z)=\phi_{\infty} z^{2}$, this is the equation found in the original linear soft wall model [10]. This equation can be solved in terms of the associated Laguerre polynomials. The normalized solutions can be written as [10]

$$
v_{n}(z)=\sqrt{\frac{2}{n+1}} \phi_{\infty} z^{2} L_{n}^{1}\left(\phi_{\infty} z^{2}\right) \quad n=0,1, \ldots,
$$

and the corresponding masses take the form

$$
m_{v_{n}}^{2}=4 \phi_{\infty}(n+1) \quad n=0,1, \ldots,
$$

which organize into a linear Regge trajectory. The vector meson decay constants in the linear soft wall model take the form

$$
F_{v^{n}}=\frac{1}{g_{5}}\left[e^{A_{s}-\Phi} \partial_{z} v_{c, n}\right]_{z \rightarrow 0}=\frac{\phi_{\infty}}{g_{5}} \sqrt{8(n+1)} .
$$

Note that $F_{v^{n}}^{2}$ also organize into a linear Regge trajectory.

For the differential equation of the scalar mode $s_{n}(z)$, all the models reduce to the linear soft wall model if we take the limits $a_{0} \rightarrow 0$ (minimal coupling) and $m_{q} \rightarrow 0$ (chiral limit). The resulting differential equation can be written as

$$
\left\{\left[\partial_{z}+3 A_{s}^{\prime}-\Phi^{\prime}\right] \partial_{z}+3 e^{2 A_{s}}\right\} s_{n}=-m_{s_{n}}^{2} s_{n}
$$

with normalized solutions [12]

$s_{n}(z)=\sqrt{\frac{2}{n+1}} \phi_{\infty} z^{3} L_{n}^{1}\left(\phi_{\infty} z^{2}\right) \quad n=0,1, \ldots$

and masses

$$
m_{s_{n}}^{2}=\phi_{\infty}(4 n+6) \quad n=0,1, \ldots
$$

also organized into a linear Regge trajectory. The scalar meson decay constant in the linear soft wall model takes the form

$$
F_{s^{n}}=\zeta\left[z e^{3 A_{s}-\Phi} \partial_{z} s_{n}\right]_{z \rightarrow 0}=\zeta \phi_{\infty} \sqrt{18(n+1)}
$$

Note that $F_{s^{n}}^{2}$ also organize into a linear Regge trajectory.

\section{APPENDIX E: LINEAR SOFT WALL MODEL WITH NEGATIVE DILATON}

The negative dilaton, proposed in Refs. [52,53], was introduced as an alternative to describe hadronic spectrum and spontaneous chiral symmetry breaking in the holographic soft wall model for QCD. However, there is a debate about the consequences arising with the negative dilaton profile. It was shown by the authors of Ref. [54], that the negative dilaton will drive to the emergence of one massless scalar (unphysical) state in the spectrum of the vector mesons; outside of that, the negative dilaton does not allow us to get the generalized relation valid for higher spin fields

$$
m_{n, S}^{2}=4 \phi_{\infty}(n+S),
$$

where $n$ is the radial excitation number and $S$ is the spin. In the negative dilaton scenario, $\Phi=\Theta \phi_{\infty} z^{2}$, where $\Theta$ is a negative number. For higher spin fields, the corresponding results with negative dilaton become 


$$
m_{n, S}^{2}=4|\Theta| \phi_{\infty}(n+1),
$$

representing unphysical states. Despite these caveats, in the following, we present the derivation of the solution of the tachyon field considering the negative dilaton; then, we will show that the description of spontaneous chiral symmetry breaking is possible within this scenario. The negative dilaton is given by

$$
\Phi=-\phi_{\infty} z^{2}
$$

where we have set $\Theta=-1$. To calculate the solution of the tachyon differential equation (14), we write it in the form (setting $a=\Phi$ and $\lambda=0$ )

$$
\left(z^{2} \partial_{z}^{2}-\left(3-2 \phi_{\infty} z^{2}\right) z \partial_{z}-m_{X}^{2}\right) v(z)=0 .
$$

Introducing the new variable $x=\phi_{\infty} z^{2}$, the last equation becomes

$$
\left(x^{2} \partial_{x}^{2}-(1-x) x \partial_{x}-\frac{m_{X}^{2}}{4}\right) v(x)=0
$$

This equation can be rewritten in a convenient form using the transformation $v(x)=x^{\beta} \mathcal{V}(x)$,

$$
\left(x \partial_{x}^{2}+(2 \beta-1+x) \partial_{x}+\beta+\frac{\beta^{2}-2 \beta-\frac{m_{X}^{2}}{4}}{x}\right) \mathcal{V}=0
$$

The last differential equation becomes a Kummer-like differential equation under the condition

$$
\beta^{2}-2 \beta-\frac{m_{X}^{2}}{4}=0, \rightarrow \beta=1 \pm \sqrt{1+\frac{m_{X}^{2}}{4}}=\frac{\Delta_{ \pm}}{2} .
$$

To get the standard form of Kummer's differential equation, we must introduce an additional transformation in (E6), $\hat{v}(x)=e^{-\alpha x} \mathcal{V}(x)$, such that the differential equation becomes

$$
\begin{aligned}
& \left(x \partial_{x}^{2}+(2 \beta-1+(1-2 \alpha) x) \partial_{x}+\beta+\alpha(1-2 \beta)\right. \\
& \quad+\alpha(\alpha-1) x) \hat{v}(x)=0 .
\end{aligned}
$$

Kummer's differential equation is obtained by setting $\alpha=1$,

$$
\left(x \partial_{x}^{2}+(2 \beta-1-x) \partial_{x}+1-\beta\right) \hat{v}(x)=0 .
$$

Then, the general solution is a linear combination of $U(a, b, ; x)$ and $x^{1-b} M(1+a-b, 2-b ; x)$, the Tricomi and Kummer functions, respectively. From Eq. (E9), we identify $a=\beta-1$ and $b=2 \beta-1^{4}$; after restoring the original function and variable, $v(z)$, we get the complete solution

$$
\begin{aligned}
v= & e^{-\phi_{\infty} z^{2}}\left(\mathcal{C}_{1} z^{2 \beta} U\left(\beta-1,2 \beta-1 ; \phi_{\infty} z^{2}\right)\right. \\
& \left.+\mathcal{C}_{3} z^{2(2-\beta)} M\left(1-\beta, 3-2 \beta ; \phi_{\infty} z^{2}\right)\right),
\end{aligned}
$$

where $\mathcal{C}_{1}$ and $\mathcal{C}_{3}$ are constants. Plugging $m_{X}^{2}=-3$ in Eq. (E7), we get $\Delta_{-}=1, \Delta_{+}=3$, and $\beta=1 / 2$. Thus, we may expand the solution close to the boundary and read off the leading coefficients, i.e., the source and vacuum expectation value,

$$
\begin{aligned}
v= & c_{1} z+\left(\sqrt{\pi} C_{0} \phi_{\infty}^{3 / 2}-\frac{c_{1} \phi_{\infty}}{2}(1+\gamma)\right) z^{3} \\
& -\frac{c_{1} \phi_{\infty}}{2} z^{3} \log \left(\frac{\phi_{\infty} z^{2}}{4}\right)+\cdots
\end{aligned}
$$

where we have defined $\mathcal{C}_{1}=\sqrt{\pi} c_{1}, \mathcal{C}_{3}=\phi_{\infty}^{3 / 2} C_{0}$, and $\gamma$ is Euler's constant. In turn, considering the asymptotic form of the Tricomi and Kummer functions in the IR, $U(a, b ; x) \sim x^{-a}$ and $M(a, b ; x) \sim e^{x} x^{a-b}$, the leading terms are $U \sim z$ and $M \sim e^{\phi_{\infty} z^{2}} z^{-3}$; then, expanding $v(z)$ in the IR,

$$
\begin{aligned}
v= & C_{0}\left(1+\frac{3}{4 \phi_{\infty} z^{2}}+\frac{45}{32 \phi_{\infty}^{2} z^{4}}+\cdots\right) \\
& +C_{2} e^{-\phi_{\infty} z^{2}}\left(\phi_{\infty} z^{2}+\frac{1}{4}-\frac{3}{32 \phi_{\infty} z^{2}}+\cdots\right),
\end{aligned}
$$

where we have introduced the parameter $C_{2}=c_{1}$ $\sqrt{\pi} / \sqrt{\phi_{\infty}}$. In AdS/CFT, the asymptotic expansion of the field close to the boundary gives us the source and vacuum expectation value of the corresponding dual operator. Thus, the general form of the asymptotic expansion of the tachyon is given by $v=c_{1} z+c_{3} z^{3}$, where $c_{1} \propto$ $m_{q}$ (quark mass) and $c_{3} \propto\langle\bar{q} q\rangle$ (chiral condensate). From Eq. (E11), we may extract the constant $c_{3}$,

$$
c_{3}=C_{0} \sqrt{\pi} \phi_{\infty}^{3 / 2}-\frac{c_{1} \phi_{\infty}}{2}(1+\gamma) .
$$

As can be seen, the parameter $c_{3}$ depends on two independent parameters $C_{0}$ and $c_{1}$. In the chiral limit, $c_{1}=0$, and $c_{3} \neq 0$ because $C_{0} \neq 0$; hence, $\langle\bar{q} q\rangle \neq 0$. Therefore, the scenario with a negative dilaton allows a description of spontaneous chiral symmetry breaking.

\footnotetext{
${ }^{4}$ Do not confuse the constants $a$ and $b$ with the nonminimal couplings defined in previous sections.
} 
However, there is, at least, one issue that called our attention. The Hamiltonian depends on the derivative of the tachyon field in the IR (see, for instance, Ref. [51]), and it will diverge because of the presence of $C_{0}$ in Eq. (E12). This suggest that $C_{0}$ must be zero in order to get an finite Hamiltonian in the IR. Finally, it is worth mentioning that a solution equivalent to Eq. (E10) was obtained in Ref. [53].

\section{APPENDIX F: NONLINEAR SOFT WALL WITH A NEGATIVE DILATON}

Let us solve the nonlinear differential equation of the tachyon considering a negative dilaton,

$$
\left(z^{2} \partial_{z}^{2}-\left(3-2 \phi_{\infty} z^{2}\right) z \partial_{z}-m_{X}^{2}-\frac{\lambda}{2} v^{2}(z)\right) v(z)=0 .
$$

It is expected that the presence of the nonlinear term will change the solutions obtained in Eq. (E10). However, we may solve the nonlinear differential equation asymptotically. Thus, the asymptotic solution close to the boundary may be determined through the ansatz

$$
\begin{aligned}
v= & c_{1} z+d_{3} z^{3} \ln \left(\frac{\phi_{\infty} z^{2}}{4}\right)+c_{3} z^{3}+d_{5} z^{5} \ln \left(\frac{\phi_{\infty} z^{2}}{4}\right) \\
& +c_{5} z^{5}+\cdots,
\end{aligned}
$$

where

$$
\begin{aligned}
& d_{3}=\frac{c_{1}}{8}\left(c_{1}^{2} \lambda-4 \phi_{\infty}\right), \quad d_{5}=\frac{3 c_{1}}{128}\left(c_{1}^{2} \lambda-4 \phi_{\infty}\right)^{2}, \\
& c_{5}=\frac{1}{256}\left(c_{1}^{2} \lambda-4 \phi_{\infty}\right)\left(48 c_{3}-9 c_{1}^{3} \lambda+20 c_{1} \phi_{\infty}\right) .
\end{aligned}
$$

As can be seen, the nonlinear term contributes to the coefficient $d_{3}$, while $c_{1}$ and $c_{3}$ are independent parameters. Note that form $c_{1}=\left(4 \phi_{\infty} / \lambda\right)^{1 / 2}$, the logarithm contributions and higher-order coefficients in (F3) vanish, reducing the asymptotic solution to

$$
v=\sqrt{\frac{4 \phi_{\infty}}{\lambda}} z+c_{3} z^{3}
$$

This asymptotic solution suggests that the parameter $c_{1}$ is fixed once we know the parameters $\phi_{\infty}$ and $\lambda$. Nevertheless, this result is strange since $c_{1}$ is related to the quark mass in the dual field theory, $c_{1} \propto m_{q}$.

In turn, the asymptotic solution in the IR may be calculated by changing the variable $y=1 / z$. Then, Eq. (F1) becomes $\left[\left(y \partial_{y}\right)^{2}+2\left(2-\phi_{\infty} y^{-2}\right)\left(y \partial_{y}\right)+3\right] v-\frac{\lambda}{2} v^{3}=0$.

Considering the power series ansatz, the asymptotic solution is given by

$$
v=C_{0}+\frac{C_{2}}{z^{2}}+\frac{C_{4}}{z^{4}}+\cdots,
$$

where

$$
C_{2}=\frac{C_{0}}{8 \phi_{\infty}}\left(6-C_{0}^{2} \lambda\right), \quad C_{4}=\frac{3 C_{0}}{128 \phi_{\infty}^{2}}\left(C_{0}^{2} \lambda-10\right)\left(C_{0}^{2} \lambda-6\right) .
$$

It is interesting to realize that when $C_{0}=\sqrt{6 / \lambda}$, the coefficients $C_{2}, C_{4}, \cdots$, reduce to zero, with $v=\sqrt{6 / \lambda}$ remaining as the exact solution. This solution is also an exact solution of the differential equation (F5). The complete expansion in $v$ should be considered for arbitrary $C_{0}$. After implementing the numerical solution, using as the "initial condition" the asymptotic solution in the IR, then integrating to the UV, we obtained unstable solutions, highly sensitive to the change of the domain of integration. As we are looking for stable solutions, we do not present those results in this paper.

\section{APPENDIX G: HOLOGRAPHIC DERIVATION OF THE GOR RELATION: TOY MODEL}

In this section, we propose a toy model to get the GellMann-Oakes-Renner relation. We start by building a simple function for the tachyon which takes into account the asymptotic solutions in the UV and IR. To simplify the analysis, we consider $b=\phi_{\infty} z^{2}$ with $\phi_{\infty}=1$. Then, we consider the most simple smooth function interpolating between the asymptotic solution in the UV, $v=c_{1} z+c_{3} z^{3}$, and IR, $v=C_{0}$, which is given by

$$
v=\frac{c_{1} z+c_{3} z^{3}}{1+\frac{c_{3}}{C_{0}} z^{3}} .
$$

As can be seen, this simple function contains the relevant parameters arising in the UV and IR. We can check the UV expansion, which is given by

$$
v=c_{1} z+c_{3} z^{3}+\mathcal{O}\left(z^{4}\right), \quad z \rightarrow 0,
$$

while its IR expansion is given by

$$
v=C_{0}+\mathcal{O}\left(z^{-2}\right), \quad z \rightarrow \infty .
$$

Then, plugging (G1) in Eq. (71), one can integrate 

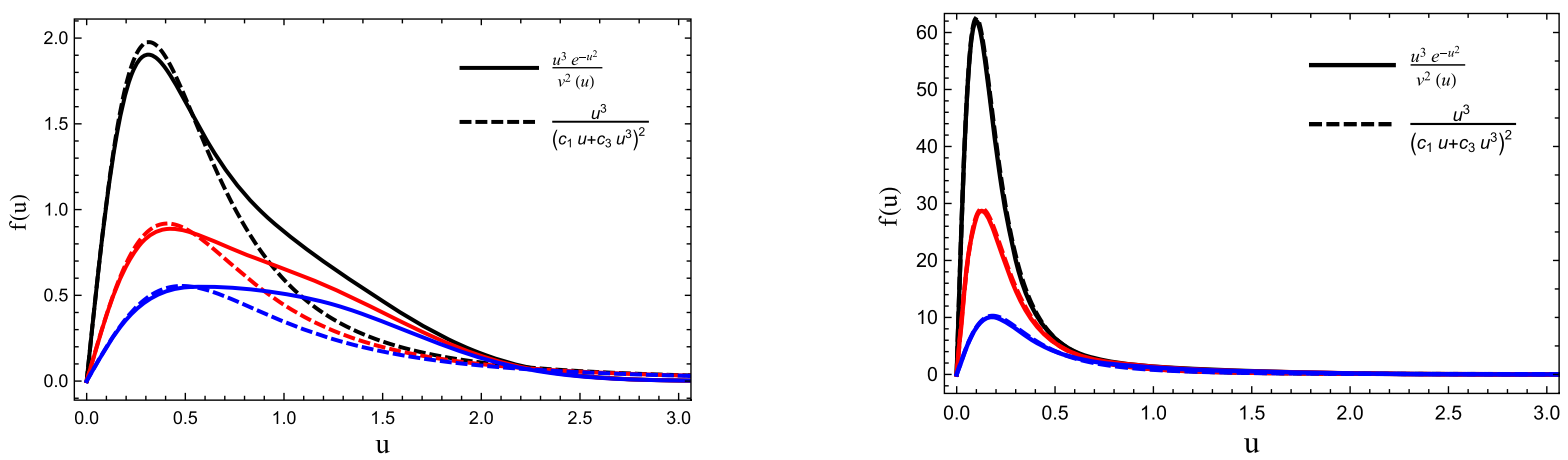

FIG. 26. Left: the kernel of the integral (solid lines) against the Gaussian function (dashed lines) for selected values of $c_{1}, c_{1}=0.7$ (blue), 0.5 (red), 0.3 (black). Right: the kernel of the integral (solid lines) against the Gaussian function (dashed lines) for selected values of $c_{1}=0.1$ (blue), 0.05 (red), 0.03 (black).

$$
\begin{aligned}
\int_{0}^{\infty} \frac{u^{3} e^{-u^{2}}}{v(u)^{2}} d u= & \frac{1}{2 c_{1} c_{3}}+\left(\frac{c_{1}^{3}}{2 C_{0}^{2} c_{3}^{2}}+\frac{1}{2 c_{3}^{2}}-\frac{3 c_{1}^{2}}{2 C_{0}^{2} c_{3}^{2}}\right) e^{c_{1} / c_{3}} \operatorname{Ei}\left(-\frac{c_{1}}{c_{3}}\right)-\frac{c_{1}^{2}}{2 C_{0} c_{3}^{2}} \\
& +\frac{1}{2 C_{0}^{2}}-\frac{c_{1}}{C_{0}^{2} c_{3}}+\frac{c_{1} \sqrt{\pi}}{C_{0} c_{3}^{2}}+\frac{\sqrt{\pi}}{C_{0} c_{3}}-\left(\frac{3 c_{1}^{3 / 2} c_{3}^{3 / 2}}{2 C_{0} c_{1} c_{3}^{3}}+\frac{2 c_{1}^{5 / 2} c_{3}^{1 / 2}}{2 C_{0} c_{1} c_{3}^{3}}\right) \pi e^{c_{1} / c_{3}} \operatorname{Erfc}\left(\sqrt{\frac{c_{1}}{c_{3}}}\right)
\end{aligned}
$$

where $\operatorname{Ei}(x)$ is the exponential integral function and $\operatorname{Erfc}(x)$ the complementary error function. So far, we did not say anything about the parameters $c_{1}, c_{3}$, and $C_{0}$; this means that the last results is valid for arbitrary values of these parameters. However, as we want to investigate the GOR relation, we must consider the regime of small $c_{1}$ parameter. Thus, the leading term of Eq. (G4) is

$$
\int_{0}^{\infty} \frac{u^{3} e^{-u^{2}}}{v(u)^{2}} d u=\frac{1}{2 c_{1} c_{3}}
$$

which is equal to Eq. (71). Plugging this result in (70),

$$
\frac{f_{\pi}^{2} m_{\pi}^{2}}{2 c_{1} c_{3}}=1
$$

yields to the GOR relation.

An alternative way to show that the result (G5) is consistent is plotting the kernel function of the integral, left side of Eq. (G5), and investigating the behavior of this function in the region of small $c_{1}$. Thus, we define

$$
f(u)=\frac{u^{3} e^{-u^{2}}}{v(u)^{2}}
$$

The plot of this function compared against the Gaussian function $\frac{u^{3}}{\left(c_{1} u+c_{3} u^{3}\right)^{2}}$ is displayed in Fig. 26. As can be seen, in the left panel, we compare the behavior of these functions by changing the value of $c_{1}$, while the right panel shows the same functions for small values of $c_{1}$.

From Fig. 26, we may conclude that the kernel of the integral is approximately equal to the Gaussian function in the regime of small $c_{1}$,

$$
\frac{u^{3} e^{-u^{2}}}{\left(\frac{c_{1} u+c_{3} u^{3}}{1+\frac{c_{3}}{c_{0}} u^{3}}\right)^{2}} \approx \frac{u^{3}}{\left(c_{1} u+c_{3} u^{3}\right)^{2}}
$$

This approximation gets better decreasing $c_{1}$. Therefore, we can replace the Gaussian function in the integral, getting

$$
\int_{0}^{\infty} \frac{u^{3} d u}{\left(c_{1} u+c_{3} u^{3}\right)^{2}}=\frac{1}{2 c_{1} c_{3}}
$$

which is the result we got previously in (G5) and (71). 
[1] J. Goldstone, A. Salam, and S. Weinberg, Broken symmetries, Phys. Rev. 127, 965 (1962).

[2] M.E. Peskin and D. V. Schroeder, An Introduction to Quantum Field Theory (Addison-Wesley, Reading, MA, 1995).

[3] M. Maggiore, A Modern Introduction to Quantum Field Theory (Oxford University Press, New York, 2005).

[4] G. P. Engel, L. Giusti, S. Lottini, and R. Sommer, Chiral Symmetry Breaking in QCD with Two Light Flavors, Phys. Rev. Lett. 114, 112001 (2015).

[5] J. M. Maldacena, The large N limit of superconformal field theories and supergravity, Adv. Theor. Math. Phys. 2, 231 (1998).

[6] S. S. Gubser, I. R. Klebanov, and A. M. Polyakov, Gauge theory correlators from noncritical string theory, Phys. Lett. B 428, 105 (1998).

[7] E. Witten, Anti-de Sitter space and holography, Adv. Theor. Math. Phys. 2, 253 (1998).

[8] J. Erlich, E. Katz, D. T. Son, and M. A. Stephanov, QCD and a Holographic Model of Hadrons, Phys. Rev. Lett. 95, 261602 (2005).

[9] L. Da Rold and A. Pomarol, Chiral symmetry breaking from five dimensional spaces, Nucl. Phys. B721, 79 (2005).

[10] A. Karch, E. Katz, D. T. Son, and M. A. Stephanov, Linear confinement and AdS/QCD, Phys. Rev. D 74, 015005 (2006).

[11] K. Ghoroku, N. Maru, M. Tachibana, and M. Yahiro, Holographic model for hadrons in deformed AdS(5) background, Phys. Lett. B 633, 602 (2006).

[12] P. Colangelo, F. De Fazio, F. Giannuzzi, F. Jugeau, and S. Nicotri, Light scalar mesons in the soft-wall model of AdS/ QCD, Phys. Rev. D 78, 055009 (2008).

[13] T. Gherghetta, J. I. Kapusta, and T. M. Kelley, Chiral symmetry breaking in the soft-wall AdS/QCD model, Phys. Rev. D 79, 076003 (2009).

[14] Z. Abidin and C. E. Carlson, Strange hadrons and kaon-topion transition form factors from holography, Phys. Rev. D 80, 115010 (2009).

[15] H. R. Grigoryan and A. V. Radyushkin, Pion form-factor in chiral limit of hard-wall AdS/QCD model, Phys. Rev. D 76, 115007 (2007).

[16] A. Vega and I. Schmidt, Scalar hadrons in $\operatorname{AdS}(5) \times \mathrm{S} * * 5$, Phys. Rev. D 78, 017703 (2008).

[17] H. J. Kwee and R. F. Lebed, Pion form-factors in holographic QCD, J. High Energy Phys. 01 (2008) 027.

[18] H. J. Kwee and R. F. Lebed, Pion form factor in improved holographic QCD backgrounds, Phys. Rev. D 77, 115007 (2008).

[19] K. Chelabi, Z. Fang, M. Huang, D. Li, and Y.-L. Wu, Chiral phase transition in the soft-wall model of AdS/QCD, J. High Energy Phys. 04 (2016) 036.

[20] L.-X. Cui, Z. Fang, and Y.-L. Wu, Infrared-improved softwall AdS/QCD model for mesons, Eur. Phys. J. C 76, 22 (2016).

[21] Y.-Q. Sui, Y.-L. Wu, Z.-F. Xie, and Y.-B. Yang, Prediction for the mass spectra of resonance mesons in the soft-wall AdS/QCD with a modified 5D metric, Phys. Rev. D 81, 014024 (2010).
[22] Z. Fang, Y.-L. Wu, and L. Zhang, Chiral phase transition and meson spectrum in improved soft-wall AdS/QCD, Phys. Lett. B 762, 86 (2016).

[23] A. Ballon-Bayona, G. a. Krein, and C. Miller, Decay constants of the pion and its excitations in holographic QCD, Phys. Rev. D 91, 065024 (2015).

[24] A. Ballon-Bayona, G. Krein, and C. Miller, Strong couplings and form factors of charmed mesons in holographic QCD, Phys. Rev. D 96, 014017 (2017).

[25] M. A. Martin Contreras, E. Folco Capossoli, D. Li, A. Vega, and H. Boschi-Filho, Pion form factor from an AdS deformed background, arXiv:2104.04640.

[26] X. Cao, S. Qiu, H. Liu, and D. Li, Thermal properties of light mesons from holography, J. High Energy Phys. 08 (2021) 005.

[27] E. Folco Capossoli, M. A. Martín Contreras, D. Li, A. Vega, and H. Boschi-Filho, Hadronic spectra from deformed AdS backgrounds, Chin. Phys. C 44, 064104 (2020).

[28] E. Kiritsis and F. Nitti, On massless 4D gravitons from asymptotically AdS(5) space-times, Nucl. Phys. B772, 67 (2007).

[29] U. Gursoy, E. Kiritsis, and F. Nitti, Exploring improved holographic theories for QCD: Part II, J. High Energy Phys. 02 (2008) 019.

[30] S. S. Gubser, A. Nellore, S. S. Pufu, and F. D. Rocha, Thermodynamics and Bulk Viscosity of Approximate Black Hole Duals to Finite Temperature Quantum Chromodynamics, Phys. Rev. Lett. 101, 131601 (2008).

[31] D. Li and M. Huang, Dynamical holographic QCD model for glueball and light meson spectra, J. High Energy Phys. 11 (2013) 088.

[32] A. Ballon-Bayona, H. Boschi-Filho, L. A. H. Mamani, A. S. Miranda, and V. T. Zanchin, Effective holographic models for QCD: Glueball spectrum and trace anomaly, Phys. Rev. D 97, 046001 (2018).

[33] A. Ballon-Bayona, H. Boschi-Filho, L. A. H. Mamani, A. S. Miranda, and V.T. Zanchin, An effective holographic approach to QCD, in Proceedings of the 14th International Workshop on Hadron Physics (2018), 4 [arXiv:1804.01579].

[34] A. Ballon-Bayona, L. A. H. Mamani, A. S. Miranda, and V. T. Zanchin, Effective holographic models for QCD: Thermodynamics and viscosity coefficients, Phys. Rev. D 104, 046013 (2021).

[35] I. R. Klebanov and M. J. Strassler, Supergravity and a confining gauge theory: Duality cascades and chi SB resolution of naked singularities, J. High Energy Phys. 08 (2000) 052.

[36] J. M. Maldacena and C. Nunez, Towards the Large N Limit of Pure $N=1$ SuperYang-Mills, Phys. Rev. Lett. 86, 588 (2001).

[37] A. Karch and E. Katz, Adding flavor to AdS/CFT, J. High Energy Phys. 06 (2002) 043.

[38] T. Sakai and S. Sugimoto, Low energy hadron physics in holographic QCD, Prog. Theor. Phys. 113, 843 (2005).

[39] F. Bigazzi and A. L. Cotrone, Holographic QCD with dynamical flavors, J. High Energy Phys. 01 (2015) 104.

[40] J. D. Edelstein and R. Portugues, Gauge/string duality in confining theories, Fortschr. Phys. 54, 525 (2006). 
[41] J. Erdmenger, N. Evans, I. Kirsch, and E. Threlfall, Mesons in gauge/gravity duals-A review, Eur. Phys. J. A 35, 81 (2008).

[42] C. Nunez, A. Paredes, and A. V. Ramallo, Unquenched flavor in the gauge/gravity correspondence, Adv. High Energy Phys. 2010, 196714 (2010).

[43] N. J. Evans and J. P. Shock, Chiral dynamics from AdS space, Phys. Rev. D 70, 046002 (2004).

[44] T. Sakai and S. Sugimoto, More on a holographic dual of QCD, Prog. Theor. Phys. 114, 1083 (2005).

[45] R. Casero, E. Kiritsis, and A. Paredes, Chiral symmetry breaking as open string tachyon condensation, Nucl. Phys. B787, 98 (2007).

[46] I. Iatrakis, E. Kiritsis, and A. Paredes, An AdS/QCD model from tachyon condensation: II, J. High Energy Phys. 11 (2010) 123.

[47] I. Iatrakis, E. Kiritsis, and A. Paredes, An AdS/QCD model from Sen's tachyon action, Phys. Rev. D 81, 115004 (2010).

[48] M. Jarvinen and E. Kiritsis, Holographic models for QCD in the Veneziano limit, J. High Energy Phys. 03 (2012) 002.

[49] T. Alho, N. Evans, and K. Tuominen, Dynamic AdS/QCD and the spectrum of walking gauge theories, Phys. Rev. D 88, 105016 (2013).

[50] K. Bitaghsir Fadafan, W. Clemens, and N. Evans, Holographic gauged NJL model: The conformal window and ideal walking, Phys. Rev. D 98, 066015 (2018).

[51] A. Ballon-Bayona and L. A. H. Mamani, Nonlinear realization of chiral symmetry breaking in holographic soft wall models, Phys. Rev. D 102, 026013 (2020).

[52] G. F. de Teramond and S. J. Brodsky, Light-front holography and gauge/gravity duality: The light meson and baryon spectra, Nucl. Phys. B, Proc. Suppl. 199, 89 (2010).

[53] F. Zuo, Improved soft-wall model with a negative dilaton, Phys. Rev. D 82, 086011 (2010).

[54] A. Karch, E. Katz, D. T. Son, and M. A. Stephanov, On the sign of the dilaton in the soft wall models, J. High Energy Phys. 04 (2011) 066.

[55] S. R. Coleman and E. Witten, Chiral Symmetry Breakdown in Large N Chromodynamics, Phys. Rev. Lett. 45, 100 (1980).

[56] F. Bigazzi, R. Casero, A. L. Cotrone, E. Kiritsis, and A. Paredes, Non-critical holography and four-dimensional CFT's with fundamentals, J. High Energy Phys. 10 (2005) 012.

[57] C. Charmousis, B. Gouteraux, B. S. Kim, E. Kiritsis, and R. Meyer, Effective holographic theories for low-temperature condensed matter systems, J. High Energy Phys. 11 (2010) 151.

[58] O. DeWolfe, S. S. Gubser, and C. Rosen, Dynamic critical phenomena at a holographic critical point, Phys. Rev. D 84, 126014 (2011).

[59] S. He, S.-Y. Wu, Y. Yang, and P.-H. Yuan, Phase structure in a dynamical soft-wall holographic QCD model, J. High Energy Phys. 04 (2013) 093.

[60] D. Dudal and S. Mahapatra, Thermal entropy of a quarkantiquark pair above and below deconfinement from a dynamical holographic QCD model, Phys. Rev. D 96, 126010 (2017).

[61] I. Y. Aref'eva, K. Rannu, and P. Slepov, Holographic anisotropic model for light quarks with confinement- deconfinement phase transition, J. High Energy Phys. 06 (2021) 090.

[62] D. Areán, I. Iatrakis, M. Järvinen, and E. Kiritsis, The discontinuities of conformal transitions and mass spectra of V-QCD, J. High Energy Phys. 11 (2013) 068.

[63] U. Gursoy and E. Kiritsis, Exploring improved holographic theories for QCD: Part I, J. High Energy Phys. 02 (2008) 032.

[64] P. Breitenlohner and D. Z. Freedman, Positive energy in anti-de Sitter backgrounds and gauged extended supergravity, Phys. Lett. 115B, 197 (1982).

[65] A. Cherman, T. D. Cohen, and E. S. Werbos, The chiral condensate in holographic models of QCD, Phys. Rev. C 79, 045203 (2009).

[66] C. P. Herzog, A Holographic Prediction of the Deconfinement Temperature, Phys. Rev. Lett. 98, 091601 (2007).

[67] E. V. Shuryak, The QCD Vacuum, Hadrons and Superdense Matter, Lecture Notes in Physics Vol. 71 (World Scientific, Singapore, 2004), https://doi.org/10.1142/5367.

[68] L. Giusti, F. Rapuano, M. Talevi, and A. Vladikas, The QCD chiral condensate from the lattice, Nucl. Phys. B538, 249 (1999).

[69] H. Fukaya, S. Aoki, S. Hashimoto, T. Kaneko, J. Noaki, T. Onogi, and N. Yamada (JLQCD Collaboration), Determination of the Chiral Condensate from $(2+1)$-Flavor Lattice QCD, Phys. Rev. Lett. 104, 122002 (2010); 105, 159901(E) (2010).

[70] C. McNeile, A. Bazavov, C. T. H. Davies, R. J. Dowdall, K. Hornbostel, G. P. Lepage, and H. D. Trottier, Direct determination of the strange and light quark condensates from full lattice QCD, Phys. Rev. D 87, 034503 (2013).

[71] P. Zyla et al. (Particle Data Group), Review of particle physics, Prog. Theor. Exp. Phys. 2020, 083C01 (2020).

[72] T. M. Kelley, S. P. Bartz, and J. I. Kapusta, Pseudoscalar mass spectrum in a soft-wall model of AdS/QCD, Phys. Rev. D 83, 016002 (2011).

[73] J. F. Donoghue, E. Golowich, and B. R. Holstein, Dynamics of the Standard Model (Cambridge University Press, Cambridge, England, 2014), Vol. 2.

[74] O. Y. A. Gokalp and Y. Sarac, Scalar $a_{0}$-meson contributions to radiative $\omega \rightarrow \pi^{0} \eta \gamma$ and $\rho^{0} \rightarrow \pi^{0} \eta \gamma$ decays, Eur. Phys. J. C 22, 327 (2001).

[75] N. Isgur, C. Morningstar, and C. Reader, The $a_{1}$ in $\tau$ decay, Phys. Rev. D 39, 1357 (1989).

[76] Y. Kinar, E. Schreiber, and J. Sonnenschein, Q anti-Q potential from strings in curved space-time: Classical results, Nucl. Phys. B566, 103 (2000).

[77] L. A. H. Mamani, A. S. Miranda, H. Boschi-Filho, and N.R.F. Braga, Vector meson quasinormal modes in a finite-temperature AdS/QCD model, J. High Energy Phys. 03 (2014) 058.

[78] A. S. Miranda, C. A. Ballon Bayona, H. Boschi-Filho, and N. R. F. Braga, Black-hole quasinormal modes and scalar glueballs in a finite-temperature AdS/QCD model, J. High Energy Phys. 11 (2009) 119.

[79] C. A. B. Bayona, H. Boschi-Filho, and N. R. F. Braga, Deep inelastic scattering off a plasma with flavour from D3-D7 brane model, Phys. Rev. D 81, 086003 (2010). 
[80] L. A. H. Mamani, A. S. Miranda, and V. T. Zanchin, Melting of scalar mesons and black-hole quasinormal modes in a holographic QCD model, Eur. Phys. J. C 79, 435 (2019).

[81] S. P. Bartz and T. Jacobson, Chiral phase transition and meson melting from AdS/QCD, Phys. Rev. D 94, 075022 (2016).
[82] F. Bigazzi, A. L. Cotrone, J. Mas, A. Paredes, A. V. Ramallo, and J. Tarrio, D3-D7 quark-gluon plasmas, J. High Energy Phys. 11 (2009) 117.

[83] F. Bigazzi, A. L. Cotrone, and J. Tarrio, Hydrodynamics of fundamental matter, J. High Energy Phys. 02 (2010) 083. 